Florida International University

FIU Digital Commons

FIU Electronic Theses and Dissertations

University Graduate School

$3-21-2013$

\title{
Structural Health Monitoring Inside Concrete and Grout Using the Wireless Identification and Sensing Platform (WISP)
}

Elicek Delgado Cepero

Florida International University, edelg014@fiu.edu

DOI: $10.25148 /$ etd.FI13042331

Follow this and additional works at: https:// digitalcommons.fiu.edu/etd

Part of the Electrical and Electronics Commons, and the Systems and Communications Commons

\section{Recommended Citation}

Delgado Cepero, Elicek, "Structural Health Monitoring Inside Concrete and Grout Using the Wireless Identification and Sensing Platform (WISP)" (2013). FIU Electronic Theses and Dissertations. 864.

https://digitalcommons.fiu.edu/etd/864 


\title{
FLORIDA INTERNATIONAL UNIVERSITY
}

Miami, Florida

\section{STRUCTURAL HEALTH MONITORING INSIDE CONCRETE AND GROUT USING THE WIRELESS IDENTIFICATION AND SENSING PLATFORM (WISP)}

\author{
A thesis submitted in partial fulfillment of the \\ requirements for the degree of \\ MASTER OF SCIENCE \\ in \\ ELECTRICAL ENGINEERING \\ by
}

Elicek Delgado-Cepero 
To: Dean Amir Mirmiran

College of Engineering and Computing

This thesis, written by Elicek Delgado-Cepero, and entitled Structural Health Monitoring Inside Concrete and Grout Using the Wireless Identification and Sensing Platform (WISP), having been approved in respect to style and intellectual content, is referred to you for judgment.

We have read this thesis and recommend that it be approved.

Leonel E. Lagos

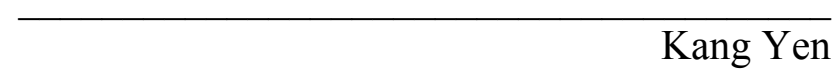

Stavros Georgakopoulos, Major Professor

Date of Defense: March 21, 2013

The thesis of Elicek Delgado-Cepero is approved.

$\begin{array}{r}\text { Dean Amir Mirmiran } \\ \text { College of Engineering and Computing } \\ \hline \begin{array}{c}\text { Dean Lakshmi N. Reddi } \\ \text { University Graduate School }\end{array}\end{array}$

Florida International University, 2013 


\section{DEDICATION}

I dedicate this work to my wonderful mother Elicet for always being the best role model, and to my beloved husband Carlos for his love and infinite support. 


\section{ACKNOWLEDGMENTS}

Intel Seattle Labs, as part of the WISP Challenge, donated the WISPs used in this research. Thanks are extended to the U.S. Department of Energy's (DOE's) Office of Environmental Management and the DOE-FIU Science \& Technology Workforce Development Program at the Applied Research Center. 
ABSTRACT OF THE MASTER'S THESIS

\title{
STRUCTURAL HEALTH MONITORING INSIDE CONCRETE AND GROUT USING \\ THE WIRELESS IDENTIFICATION AND SENSING PLATFORM (WISP)
}

\author{
by
}

Elicek Delgado-Cepero

Florida International University, 2013

Miami, Florida

\section{Professor Stavros Georgakopoulos}

This research investigates the implementation of battery-less RFID sensing platforms inside lossy media, such as, concrete and grout. Both concrete and novel grouts can be used for nuclear plant decommissioning as part of the U.S. Department of Energy's (DOE's) cleanup projects. Our research examines the following: (1) material characterization, (2) analytical modeling of transmission and propagation losses inside lossy media, (3) maximum operational range of RFID wireless sensors embedded inside concrete and grout, and (4) best positioning of antennas for achieving longer communication range between RFID antennas and wireless sensors. Our research uses the battery-less Wireless Identification and Sensing Platform (WISP) which can be used to monitor temperature, and humidity inside complex materials.

By using a commercial Agilent open-ended coaxial probe (HP8570B), the measurements of the dielectric permittivity of concrete and grout are performed. Subsequently, the measured complex permittivity is used to formulate analytical Debye models. Also, the transmission and propagation losses of a uniform plane wave inside grout are calculated. Our results show that wireless sensors will perform better in 
concrete than grout. In addition, the maximum axial and radial ranges for WISP are experimentally determined. Our work illustrates the feasibility of battery-less wireless sensors that are embedded inside concrete and grout. Also, our work provides information that can be used to optimize the power management, sampling rate, and antenna design of such sensors. 


\section{TABLE OF CONTENTS}

CHAPTER

PAGE

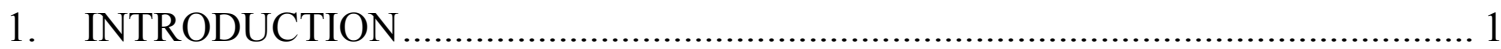

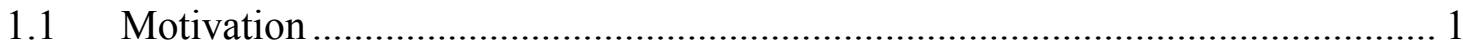

1.2 Research Purpose and Difficulties ............................................................... 2

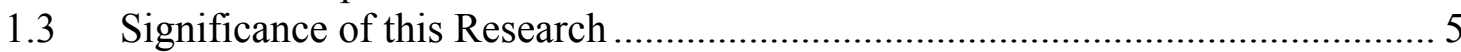

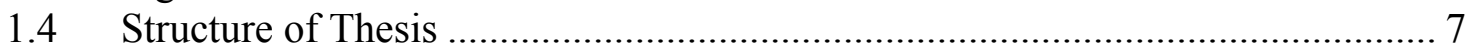

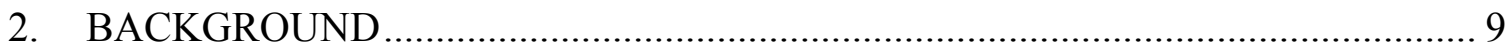

2.1 In-Situ Decommissioning of Nuclear Plants ................................................... 9

2.2 Traditional Structural Health Monitoring (SHM) Technologies......................... 11

2.3 Power Harvesting and Management of Wireless Sensors................................... 13

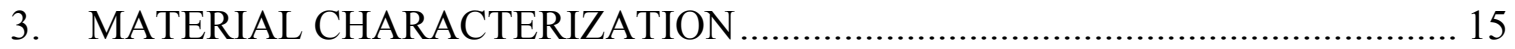

$3.1 \quad$ Electromagnetic field theory and concepts ................................................... 15

3.2 Extended Debye Model ........................................................................... 18

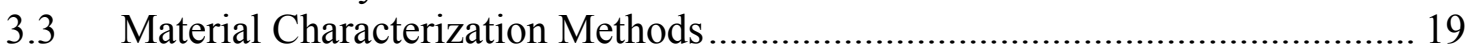

3.4 Measurements of Material Properties ............................................................ 22

4. FORMULATION OF A UNIFORM PLANE WAVE PENETRATION INSIDE

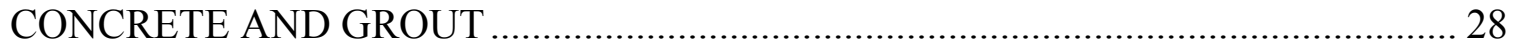

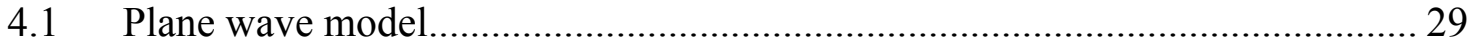

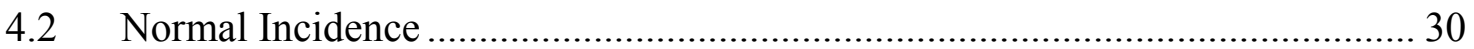

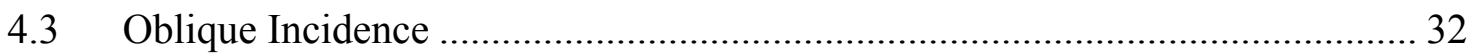

4.4 Transmission and Propagation losses inside concrete and grout ........................ 33

5. WIRELESS SENSING AND IDENTIFICATION PLATFORM............................ 47

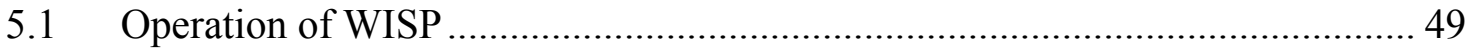

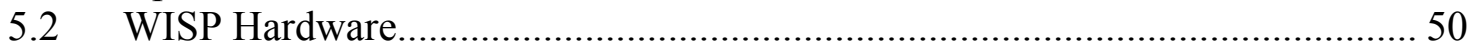

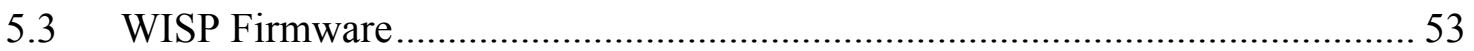

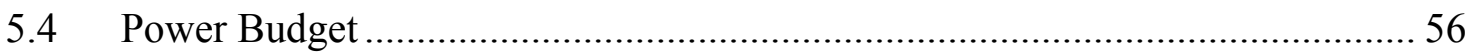

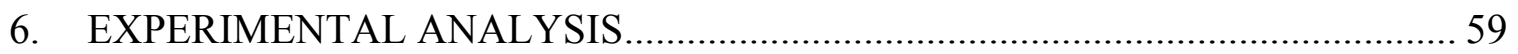

6.1 WISP maximum range of operation inside concrete and grout ......................... 61

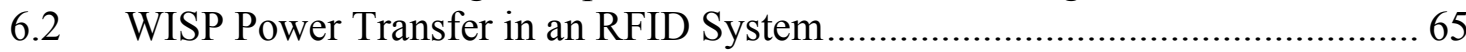

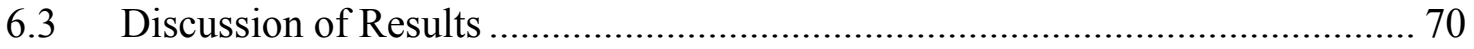

7. CONCLUSIONS AND FUTURE WORK............................................................ 71

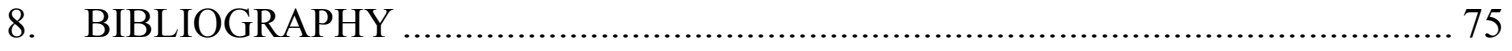




\section{LIST OF TABLES}

TABLE

PAGE

Table 4.1 Total losses for normal incidence in half-space concrete at $f=915 \mathrm{MHz} \ldots \ldots . .44$

Table 4.2 Total losses for normal incidence in half-space grout at $f=915 \mathrm{MHz} \ldots \ldots \ldots \ldots . . .44$

Table 4.3 Estimated losses in oblique incidence inside concrete at $f=915 \mathrm{MHz} \ldots \ldots \ldots . . .45$

Table 4.4 Estimated losses in oblique incidence inside grout at $f=915 \mathrm{MHz} \ldots \ldots \ldots \ldots \ldots . . .45$

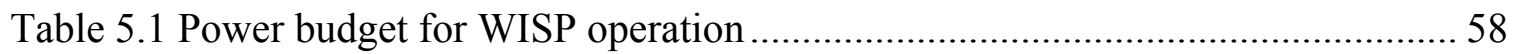

Table 6.1 Maximum radial and axial ranges of WISP inside concrete for different depths 63

Table 6.2 Maximum radial and axial ranges of WISP inside grout for different depths.. 64 


\section{LIST OF FIGURES}

FIGURE

PAGE

Figure 2.1 Cross-sectional view of a nuclear plant................................................. 10

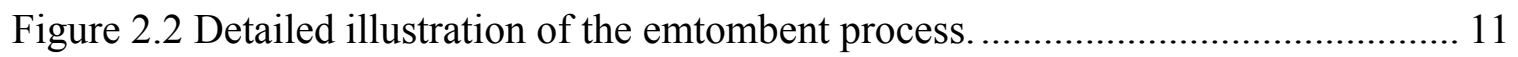

Figure 3.1 Reflection non-resonant method for complex dielectric permittivity measurements. . 21

Figure 3.2 Dimensions of the used open-ended probe............................................ 21

Figure 3.3 Calibration and measurement of dielectric properties of deionized water...... 23

Figure 3.4 Measuring grout with an open-ended probe.......................................... 23

Figure 3.5 Real part of the complex dielectric permitivity of water............................. 24

Figure 3.6 Imaginary part of the complex dielectric permitivity of water........................ 24

Figure 3.7 Complex dielectric permittivity of concrete .......................................... 25

Figure 3.8 Complex dielectric permittivity of grout.............................................. 26

Figure 4.1 Plane wave model for normal incidence in (a) half-space, and (b) finite slab

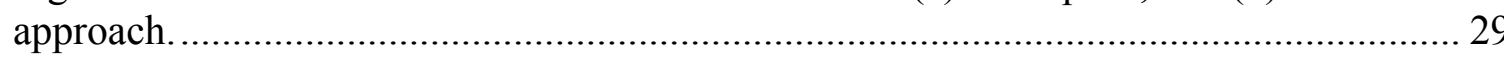

Figure 4.2 Plane wave model for oblique incidence............................................... 32

Figure 4.3 Transmission loss, $A t$, for concrete in normal incidence.............................. 34

Figure 4.4 Transmission loss, $A t$, for concrete in oblique incidence. ........................... 35

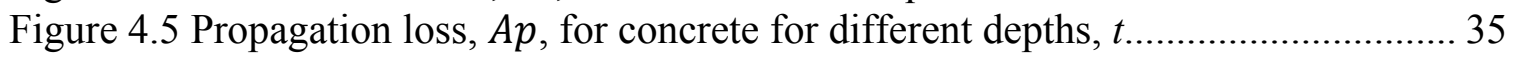

Figure 4.6 Total loss, ATOTAL for different depths, $t$, inside half-space concrete for

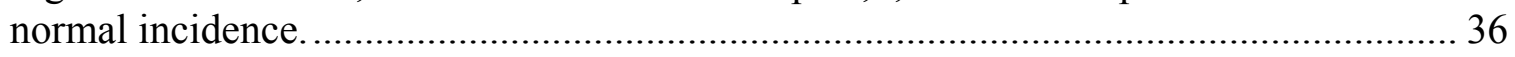

Figure 4.7 Total loss, ATOTAL for concrete slabs with different depths, $t$, for normal incidence.

Figure 4.8 Total loss, ATOTAL(II) for different depths, $t$, inside concrete in half-space case at oblique incidence. 38 
Figure 4.9 Total loss, $\operatorname{ATOTAL}(\perp)$ for different depths, $t$, inside concrete in half-space case at oblique incidence.

Figure 4.10 Transmission loss, At of grout in normal incidence..................................... 39

Figure 4.11 Transmission loss, At of grout in oblique incidence................................... 40

Figure 4.12 Propagation loss, $A p$ for grout with different depths, $t$.............................. 41

Figure 4.13 Total loss, ATOT AL for grout with different depths, $t$, in half space at normal incidence.

Figure 4.14 Total loss, ATOT AL for grout with different depths, $t$, in slab approach at normal incidence.

Figure 4.15 Total loss, ATOTAL(II) of grout with different depths, $t$, in half-space at oblique incidence.

Figure 4.16 Total loss, $\operatorname{ATOTAL}(\perp)$ of grout with different depths, $t$, in half-space at oblique incidence.

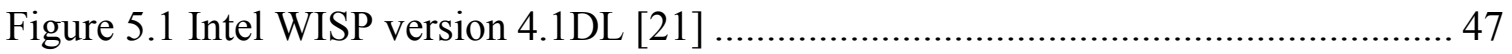

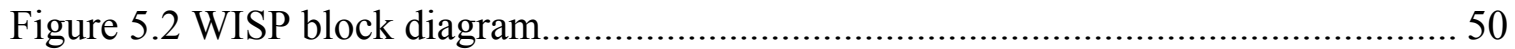

Figure 5.3 USB Key Debugger and WISP for programming. (Photo from [21])............ 54

Figure 5.4 WISP demo application for running the RFID reader...................................55

Figure 5.5 Firmware block diagram...................................................................... 56

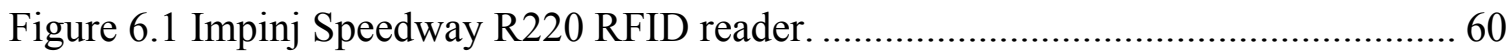

Figure 6.2 Laird Cushcraft 902-928 MHz 8 dBi Circularly Polarized Antenna............... 60

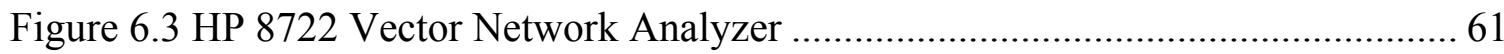

Figure 6.4 Experimental setup for measuring maximum axial range, $a$, and radial range, $r$,

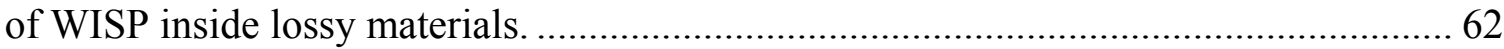

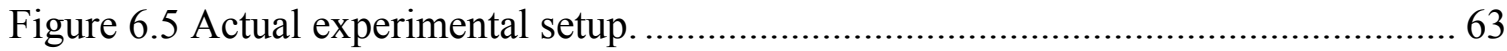

Figure 6.6 Temperature measurement with the WISP inside concrete.............................. 65 
Figure 6.7 Experimental setup for measuring the transmission coefficient $S 12$ between

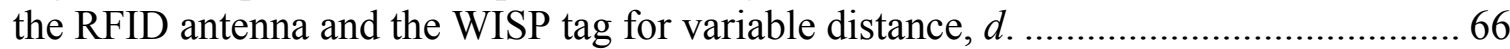

Figure 6.8 Power transfer experimental setup for the WISP inside concrete at different

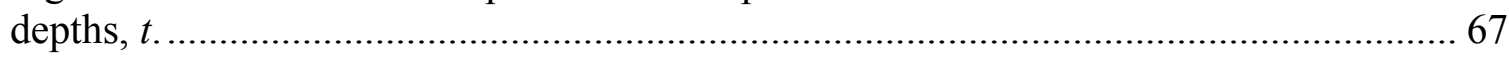

Figure 6.9 Power transfer experimental setup for the WISP inside grout at different

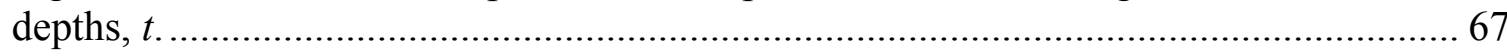

Figure 6.10 Coupling between the RFID antenna and the WISP in concrete at different depths, $t$, versus distance, $d$, at $f=915 \mathrm{MHz}$.

Figure 6.11 Coupling between the RFID antenna and the WISP in grout at different depths, $t$, versus distance, $d$, at $f=915 \mathrm{MHz}$. 


\section{INTRODUCTION}

\subsection{Motivation}

In the last few decades, the United States Department of Energy (DOE) has been involved in the decommissioning of industrial, radiological and nuclear facilities [1]. Internationally adopted decommissioning methods are: (1) Immediate dismantling (DECON), (2) Safe Enclosure (SAFSTOR) (delayed DECON), and (3) Entombment (ENTOMB) [2]. Methods (1) and (2) require conventional cleaning mechanisms by properly packaging, transporting, and disposing any nuclear waste inside the facility [3]. After several controls, these facilities can be reused, if applicable. However, these two methods are proven to be too costly and time consuming. In addition, in certain cases, there are no available places for disposing this nuclear waste. Therefore, option (3), entombment of nuclear facilities with concrete and/or novel grouts, is considered a viable solution.

These nuclear facilities, in most cases, are concrete structures. Therefore, the decommissioning strategy chosen by the DOE was to isolate these structures by filling them with novel building materials, such as, flowable grouts. For that matter, in current years, the DOE has been involved in investigating novel flowable filling grouts than can contain the radioactive material inside the plant's concrete walls and foundation slabs without leaking and have a long lifetime in the order of hundred years. These buildings are planned to be abandoned in place, which is significantly less expensive than the alternative, which involves the demolition of the facilities, as well as packaging, transporting, and disposal of nuclear waste. However, even though such decommissioned 
facilities will not be functional, it is necessary to monitor the behavior of concrete and grout after being poured into the structure. Consequently, a monitoring sensor system is needed in order to collect real-time data of temperature, humidity and fluid transport inside the decommissioned structure.

\section{$\underline{\text { Research Focus }}$}

The aim of this research is to study the applicability of battery-less wireless sensors or platforms inside concrete structures filled with either concrete or grout. The Wireless Identification and Sensing Platform (WISP) created by Intel Labs combining sensors, computational resources, and a wireless transceiver will be the tested device for this study [4]. The WISP will be used for real-time data collection inside concrete and grout structures. As part of this research, we will study the electromagnetic waves propagation into such materials will be studied. Analytical modes, taking into account the material losses are essential for understanding the attenuation that of wireless signals inside lossy media, such as concrete and grout. Also, experimental results regarding the maximum radial and axial operational ranges are provided in order to identify the best positioning of sensors inside such materials. Another aspect analyzed is the coupling between a commercial RFID antenna and the WISP in order to determine the optimal distance between the antennas that provides maximum efficiency. Our objective is to be able to measure temperature inside concrete or grout using battery-less sensors.

\subsection{Research Purpose and Difficulties}

The purpose of this research effort is to investigate the feasibility of structural health monitoring (SHM) for decommissioned nuclear plants using the WISP. This 
research is applicable to nuclear plants that will be filled with a flowable grout or concrete and left for many years. There are four key components for our work: (1) material characterization, (2) mathematical modeling of transmission and propagation losses inside lossy media, (3) determination of maximum operational ranges for sensor placement, and (4) determination of maximum efficiency between the antennas for improved operational range.

\section{Material Characterization}

As part of this study, material characterization techniques are reviewed. Resonant and non-resonant methods were explained regarding methodology, benefits and challenges. Different methods for measuring complex dielectric permittivity, $\varepsilon_{r}$, of materials are examined. Specifically, there are three principal methods for evaluating the complex relative dielectric permittivity of materials, such as the open-ended coaxial probe method, the transmission-line method, and the resonant-cavity method [5]. Special attention will be given to the open-ended coaxial probe method since it is the available technique for this research. The obtained knowledge about the dielectric permittivity of the materials of interest (i.e., grout and concrete) will allow the further assessment of losses that a communication signal will suffer while traveling through this media.

\section{Analytical Model for Losses Inside Lossy Media}

Following the characterization of the desired material, analytical models of transmission loss, $A_{t}$, and propagation loss, $A_{p}$, inside grout are developed. These models are developed by assuming that an ideal uniform plane wave impinges on the medium of interest (i.e, grout). Similar models have been derived for concrete in [6]. The analytical 
model contributes to the understanding of the physical interaction between electromagnetic waves and the media that contain wireless sensors. Also, such models can help in the design of optimal communication links and optimal placement of sensors.

\section{$\underline{\text { Radiofrequency Identification (RFID) }}$}

Nowadays, there are many commercial wireless sensors on the market. However, RFID wireless sensors are very attractive solutions for several monitoring applications. Radiofrequency Identification (RFID) operates on the Industrial, Scientific, and Medical (ISM) band that comprises frequency range from 902 to $928 \mathrm{MHz}$. RFID technology provides unique features, such as, identification of tags for asset tracking and inventory. Also, since RFID tags can be small and cheap, they can be used in wireless sensor networks. However, for SHM, the most advantageous feature of RFID is the backscattering modulation that is employed to establish communication between an RFID reader and its tags. Power supply is a major constraint of wireless sensors embedded inside concrete or grout. On the other hand, backscattering modulation can simultaneously supply power to and communicate with an RFID tag. Therefore, RFID tags, such as the WISP can provide distinctive real-time monitoring and computational capabilities without a battery on-board.

\section{Wireless Identification and Sensing Platform (WISP)}

The Wireless Identification and Sensing Platform (WISP) was developed through collaboration between Intel Research Labs and the University of Washington. This battery-less wireless sensing platform combines sensing with computational resources

and wireless power harvesting. Through proper power management the WISP can 
achieve a range of approximately 10 feet in air. Also, the WISP has been used for applications, such as, harvesting solar power [7], measuring strain in carbon fiber composites [8], and measuring body positions and movements during sleep [9]. However, most applications have used the WISP in air. Our work investigates the performance of WISP inside materials, such as concrete and grout.

\section{$\underline{\text { Sensor Placement }}$}

This work also examines the best possible positioning of wireless sensors inside concrete and grout. Such sensors can be utilized to collect data that can help monitor the behavior of grouts and concrete after they have been poured. Another design aspect for wireless sensors embedded in concrete/grout relates to damage of the circuitry due to the chemical reactions occurring inside concrete/grout while curing. Therefore, proper circuitry packaging must be used that will not stress the electronic components, but will protect the circuitry inside harsh environments, such as concrete and grout [10].

\subsection{Significance of this Research}

The significance of this research relates to the feasibility study of battery-less wireless sensors embedded inside concrete and grout for decommissioning of nuclear plants. A battery-free wireless structural health-monitoring platform that can monitors the curing temperature of concrete and grout, and other parameters, such as humidity and strain, is very important for a better understanding of concrete and grout behavior after they are poured inside a nuclear plant. First, the media, i.e., concrete and grout, where the sensors were embedded were studied regarding their electrical properties. Previous studies were performed for concrete for determining its dielectric permittivity [11]. Also, 
analytical models, which calculated the losses suffered by a uniform plane wave penetrating concrete, have been developed [6]. However, novel decommissioning grouts, like the one used for this study, have not been studied and their electrical properties are not known. Therefore, this study presents relevant and new knowledge regarding the following: (a) the electrical properties of novel nuclear decommissioning grouts, and (b) the amount of losses that EM waves experience when they penetrate and propagate in such grouts.

Understanding these losses allowed us to analyze and simulate the behavior of RFID tags, such as, WISP, and optimize their operational range and antenna designs. WISP was used as the monitoring platform and the following analyses were conducted: (1) determination of maximum operational ranges for sensor placement, and (2) determination of maximum efficiency between the antennas for improved operational range. The WISP is very flexible since it allows the utilization of different sensors. Specifically, WISP can measure temperature, humidity, strain, acceleration, etc. Depending on the power needed for each sensor, WISP may require circuitry changes. Although the proposed application for this research is monitoring unused nuclear reactors, this study can be applied to monitoring other structures, such as bridges, roads, and buildings.

Currently, this platform is working with single RFID tags not related to each other. However, there is undergoing work for linking more than one WISP. Since most applications for the WISP have been developed in air, this research studies the performance of WISP inside lossy media, such as concrete and grout. Our work provides important experimental data regarding the maximum communication range. In addition, 
our work analyzes the coupling between the RFID antenna and the WISP, which can be used to improve their communication link, by resulting in longer operational ranges.

\subsection{Structure of Thesis}

The application of the proposed environmental monitoring platform deals with the monitoring of environmental parameters, such as temperature, and humidity during and after concrete and grout curing. First, Chapter 2 reviews traditional methods for decommissioning nuclear plants in North America. Additionally, it reviews current technologies for performing SHM. Characteristics of wired and wireless sensors are compared in terms of the following: (1) available technologies, (2) advantages, (3) disadvantages, and (4) power harvesting techniques and challenges.

Chapters 3 and 4 cover the characterization materials in order to accurately model the signal propagation inside construction materials. Specifically, the material of grout is modeled using an extended Debye material model. Subsequently, the transmission and

propagation losses, $A_{t}$, and, $A_{p}$, respectively, were calculated. These losses are very important as they decrease the delivered power to WISPs that are embedded in concrete or grout.

Chapter 5 discusses the WISP in terms of its operation, hardware, and firmware. Also advantages and disadvantages of WISP are discussed. Finally, Chapter 6 provides the results of two experimental setups: (1) determination of maximum operational range for the WISP while embedded inside concrete and grout, and (2) determination of maximum efficiency between the RFID antenna and the WISP while embedded inside 
concrete and grout. Finally, conclusions are offered as well as future work recommendations. 


\section{BACKGROUND}

In this chapter, the in-situ or entombment decommissioning process in nuclear plants is reviewed. It covers the principles and challenges of in-situ decommissioning, and briefly reviews current technologies and power constraints.

\subsection{In-Situ Decommissioning of Nuclear Plants}

Currently, the U.S. Department of Energy (DOE) is trying to reduce the footprint of many obsolete nuclear reactors all around the country. Therefore, in-situ decomissioning of unused nuclear facilities via the filling of these structures with certain types of grout is the chosen strategy. Since most nuclear plants are located in remote areas, it was proposed to simply fill out the underground canals and demolish the remainder of the buildings. Underground grout-filled structures will be enclosed within a concrete slab for security. After several studies, it has been determined that in-situ decommmisioning is a more cost-effective technology than the disposal, packaging, and transportation of decomissioned nuclear plants. However, challenges such as understanding the temperature variations while the concrete and grout are curing were properly studied. Another key aspect for the use of a cementitious material for isolation purposes is that it is flowable and successfully cover the canals. For that purpose, the DOE has been involved in the research of novel flowable grouts that can successfully isolate these structures.

For better undestanding, Figure 2.1 shows a cross-sectional view of a nuclear plant. Inside a nuclear facility, there is a reactor vessel surrounded by underground canals, where the radioactive materials used to flow. These underground canals are solid 
concrete structures that prevent leakage of these radioactive materials into the ground water level. Canals chases that will be disposed of have an elevation of 20 feet. For analysis purposes, a simplified entombment cross-sectional view is provided in Figure

\section{2 .}

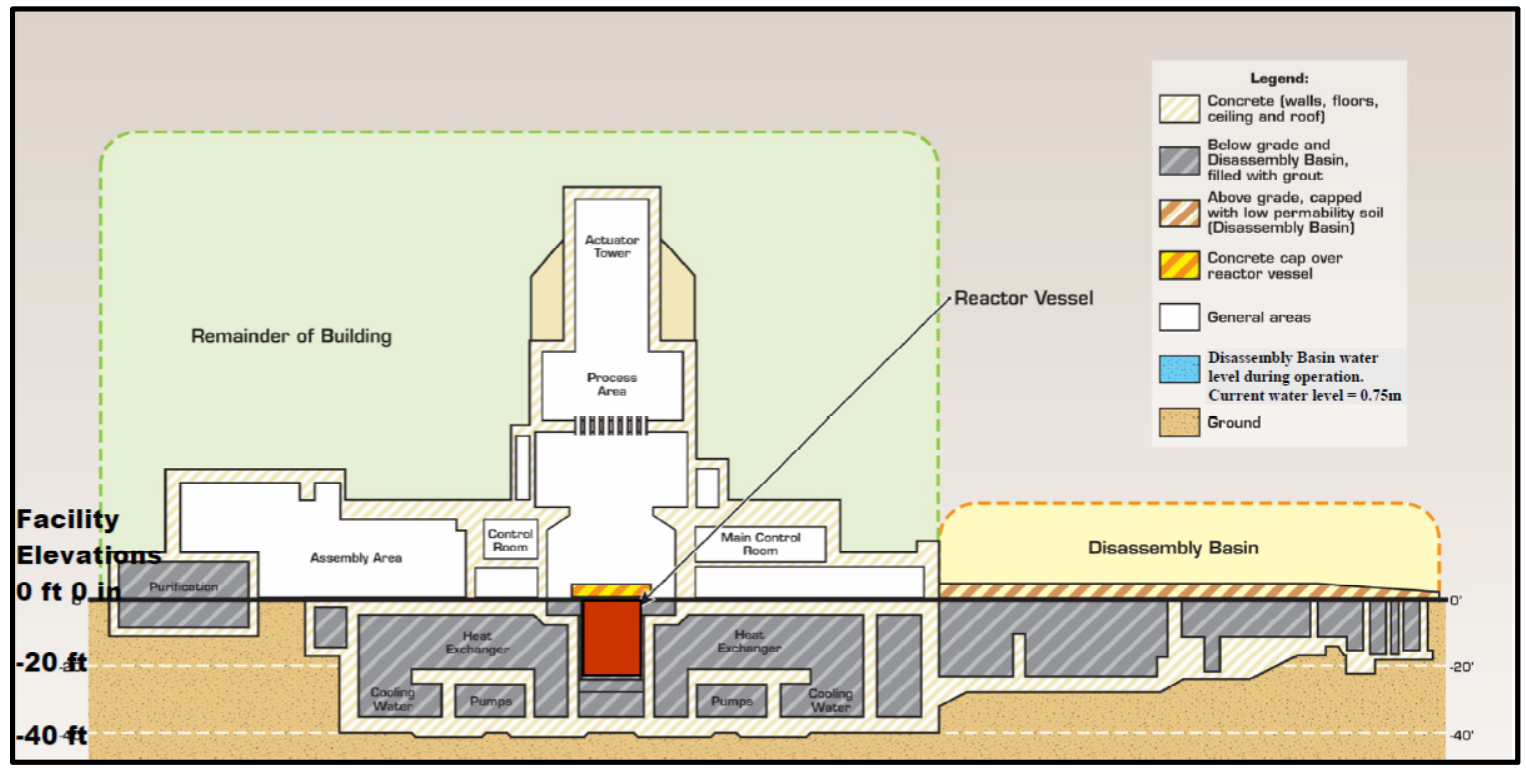

Figure 2.1 Cross-sectional view of a nuclear plant.

Current studies conducted by DOE and the Applied Research Center at Florida International University (ARC-FIU) started by studying the heat of hydration of these novel grouts. An experimental setup was developed where a test cube was filled with mixture Zero-Bleed-Flowable-Fill-No.8 Gravel Diutam Grout (PR-ZB-FF-8-D), also known as cellular grout. These novel grout ingredients are: (1) Portland Cement Type I/II, (2) Fly Ash Type F, (3) Sand (Silica) C-33, (4) Gravel (Granite) No. 8, (5) Viscocrete 2100, (6) Diutam Gum, and (7) Water [11]. 
Subsequently, wired sensors were implemented in order to evaluate temperature variation in a certain time interval, fluid level, and cracking detection. However, the wiring for the sensors was cumbersome and the power requirements of such system of wired sensors were large.

\subsection{Traditional Structural Health Monitoring (SHM) Technologies}

Throughout the years, Structural Health Monitoring (SHM) technologies have been used to assess the reliability of structures and detection of changes that can compromise their performance. Conventionally, non-destructive techniques and wired sensors are used to determine damage inside a structure. However, due to the disadvantages of wired systems, such as high installation costs, time consuming, and expensive maintenance, wireless technologies are considered a better option.

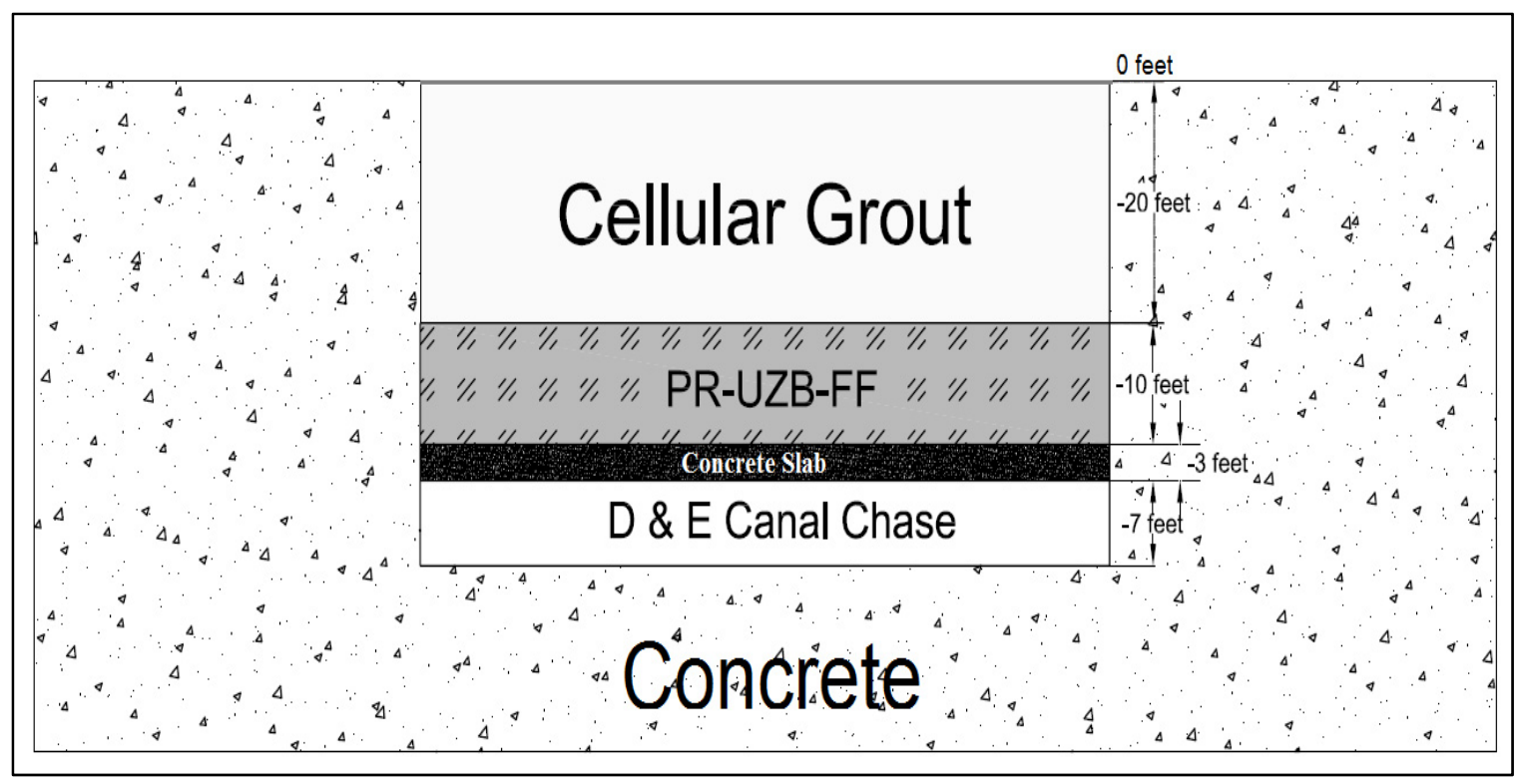

Figure 2.2 Detailed illustration of the emtombent process. 


\section{$\underline{\text { Wired Sensors and Traditional Non-Destructive Techniques }}$}

Traditional SHM technologies use wired sensors for monitoring structures. These technologies comprise of sensors attached to cables on one end, and a Data Acquisition System (DAS) on the other. Each sensor comprises of a supply power cable or battery, and a data communication system. In cases where cables are used, their performance can be compromised by corrosion and unexpected damages. Wired sensors that are installed inside concrete/grout will become inoperative once their pairing wires are compromised and their repair is not possible. Additionally, when using a large number of sensors, the amount of cables required is very large thereby making installation expensive and time consuming. Due to these limitations, wireless sensors are a better technology for SHM of concrete and grout structures.

Other techniques used to assess the state of structures include non-destructive techniques, such as visual inspections, radiography, and eddy current techniques. Piezoelectric and optical fiber sensors can be used to detect damage. Also, acousticultrasonic and guided ultrasonic waves [12] can be used. Ultrasonic waves measure stress, and eddy current techniques locate exact location of cracks inside the structures. However, such technologies are very expensive thereby preventing their broad use for SHM.

\section{$\underline{\text { Wireless Sensors }}$}

Wireless sensors are very suitable for SHM. Some of their benefits include easy installation, lower cost and small dimensions. Most common applications are SHM of roads, bridges and dams [6]. Using wireless sensors also eradicates wiring problems and 
reduces maintenance and installation costs. However, wireless sensors have drawbacks, such as limited lifetime. One promising solution to this problem involves wirelessly recharging the wireless sensors' batteries that can extend their finite lifetime.

Also, passive RFID tags are very promising for SHM since they do not use batteries. However, current passive RFID tags do not have sensing capabilities. The WISP developed by Intel Research Lab in Seattle and the University of Washington is a novel technology that combines passive RFID tags with sensors thereby enabling the development of the first battery-less and wireless SHM system.

\subsection{Power Harvesting and Management of Wireless Sensors}

As it was mentioned before, power is one of the critical factors when using wireless sensors for any application. Proper harvesting of the energy and adequate power management will determine how good the performance of monitoring devices is. Conventionally, basic wireless sensors are equipped with a battery, essential circuitry for collecting data, and sensing elements. Throughout the years, wireless sensors have improved by adding computational capabilities provided by logical blocks and microprocessors, which allow them to manage power and add different sensors. However, recharging the batteries of such sensors has remained a big constraint.

Various solutions to the power management of wireless sensors have been

offered, such as (1) power scavenging system and energy storage, (2) reduction of power consumption of sensors through the use of low power, (3) utilization of sleep modes or off-time frames between samples which minimize operation times, and (4) use of internal memories for data storage and transmitting data periodically, and not all the time. 
First, there are several sources for scavenging ambient energy. Energy can be scavenged from solar energy, wind power, thermoelectricity, physical motions or vibrations, and piezoelectric materials [13]. However, for Structural Health Monitoring applications, most popular technologies are piezoelectric accelerometers harvesting energy from vibrations in the structures. Since strain energy is one of the common phenomenon occurring inside structures most energy would come from this source.

Nevertheless, all these wireless sensors, which have either a battery or another energy source, must have a communication link between them and the data acquisition system. This requires the use of a radiofrequency signal to establish communication. Passive RFID tags provide a very promising solution for perpetual battery-less wireless sensors as they use the RF energy for both the communication and powering of such tags. This is the reason, that WISP is very suitable for the next generation SHM systems. 


\section{MATERIAL CHARACTERIZATION}

The study of material's properties is becoming increasingly important for nowadays applications. Consequently, since this research is focused on estimating the feasibility of successful communication links for wireless sensors embedded inside concrete and grout, it becomes critical to understand the interactions between electromagnetic fields and these materials. This chapter conducted a study of the properties of concrete and grout, and how they influence the propagation of RF signals through them. Specifically, the complex dielectric permittivity, $\varepsilon_{r}$, of a material provided the necessary knowledge to evaluate the behavior of electromagnetic waves while traveling through it. The electromagnetic properties of materials are also needed for designing suitable antennas for the wireless sensors that are embedded in these materials.

\subsection{Electromagnetic field theory and concepts}

In order to properly characterize the electromagnetic properties of a material, theoretical and applied concepts provided by electromagnetic field theory were reviewed. Maxwell equations [14] shown in (3.1)-(3.4) describe the behavior of electromagnetic fields. Equation (3.1) illustrates the relationship between the dielectric displacement, D, and the charge density, $\rho$. Equation (3.3) illustrates the relationship of the magnetic field, $H$, with the dielectric displacement, $D$, and the current density, $J$. Finally, equation (3.4) shows the relationship of the electric field, $E$, with the magnetic flux density, $B$.

$$
\begin{aligned}
& \nabla \cdot D=\rho \\
& \nabla \cdot B=0
\end{aligned}
$$




$$
\begin{gathered}
\nabla \times H=\partial D / \partial t+J \\
\nabla \times E=-\partial B / \partial t
\end{gathered}
$$

\section{$\underline{\text { Constitutive Parameters }}$}

The constitutive relations shown in (3.5)-(3.7) utilize the material properties, where $\varepsilon=\varepsilon^{\prime}-j \varepsilon^{\prime \prime}$ is the complex permittivity of the material, and $\mu=\mu^{\prime}-j \mu^{\prime \prime}$ is complex permeability of the material. The complex dielectric permittivity, $\varepsilon$, of a material measures the resistance that an electric field can encounter in a medium. Also, in (3.7), $\sigma$ is the conductivity of the material which means the ratio between the electric field, $E$, and the current density, $J$. Therefore, in terms of electromagnetic properties of a material, the three fundamental constitutive parameters are: (1) complex dielectric permittivity, $\varepsilon$, (2) complex dielectric permeability, $\mu$, and (3) conductivity, $\sigma$. These constitutive parameters describe the electrical properties of the material.

$$
\begin{gathered}
D=\varepsilon / E=\left(\varepsilon^{\prime}-j \varepsilon^{\prime \prime}\right) / E \\
B=\mu / H=\left(\mu^{\prime}-j \mu^{\prime \prime}\right) / H \\
J=\sigma / E
\end{gathered}
$$

For concrete and grout, since their constitutive parameters are not a function of position and they vary with frequency, $f$, they are considered to be homogeneous, and dispersive. For materials, such as, concrete and grout, the value of the conductivity, $\sigma$, is low. The complex permittivity, $\varepsilon$ relates to the relative complex dielectric permittivity, $\varepsilon_{r}$, as shown in (3.8). The relative complex dielectric permittivity, $\varepsilon_{r}$, describes the response of the material under the influence of an electromagnetic field having free space as a reference. Both, the electric and the magnetic fields, $E$, and, $H$, 
respectively, interact with materials by either storing or dissipating energy. Energy storage is related to the real part of the relative complex dielectric permittivity, $\varepsilon_{r}^{\prime}$, while the imaginary part $\varepsilon_{r}^{\prime \prime}$ is related to the energy dissipation of the electric field. The dissipated energy is absorbed by the material.

$$
\varepsilon_{r}=\varepsilon_{r}^{\prime}-j \varepsilon_{r}^{\prime \prime}=\varepsilon / \varepsilon_{0}=\varepsilon^{\prime}-j \varepsilon^{\prime \prime} / \varepsilon_{0}
$$

where $\varepsilon_{0}=8.854 \times 10^{-12}[\mathrm{~F} / \mathrm{m}]$ is the free space permittivity.

Besides the relative complex dielectric permittivity, $\varepsilon_{r}$, of a material, there other two other material parameters known as the loss tangent, $\tan \delta$, and the dielectric power factor, $\cos \delta$, as shown in (3.8) and (3.9).

$$
\begin{gathered}
\tan \delta=\varepsilon_{r}^{\prime \prime} / \varepsilon_{r}^{\prime} \\
\cos \delta=\varepsilon_{r}^{\prime \prime} / \sqrt{\left(\varepsilon_{r}^{\prime}\right)^{2}+\left(\varepsilon_{r}^{\prime \prime}\right)^{2}}
\end{gathered}
$$

\section{$\underline{\text { Propagation Parameters }}$}

The wave equations describe the propagation of RF waves in a medium and they are written in (3.11) and (3.12).

$$
\begin{aligned}
& \nabla^{2} E=j \omega \mu \sigma E-\omega^{2} \mu \varepsilon E=\gamma^{2} E \\
& \nabla^{2} H=j \omega \mu \sigma H-\omega^{2} \mu \varepsilon H=\gamma^{2} H
\end{aligned}
$$

where, $\gamma=\alpha+j \beta=j \omega \sqrt{\mu \varepsilon}=j \frac{\omega}{c} \sqrt{\mu_{r} \varepsilon_{r}}$ is the propagation constant; and $c=$ $3 \times 10^{8}[\mathrm{~m} / \mathrm{s}]$ is the velocity of wave in free space.

In a lossy medium, these parameters are expressed in term of the constitutive parameters $(\varepsilon, \mu, \sigma)$, as shown in (3.13) and (3.14).

$$
\alpha=\omega \sqrt{\mu \varepsilon}\left\{1 / 2\left[\sqrt{1+\left(\sigma / \omega \varepsilon_{r}\right)^{2}}-1\right]\right\}^{1 / 2}[N p / m]
$$




$$
\beta=\omega \sqrt{\mu \varepsilon}\left\{1 / 2\left[\sqrt{1+\left(\sigma / \omega \varepsilon_{r}\right)^{2}}+1\right]\right\}^{1 / 2}[\mathrm{rad} / \mathrm{m}]
$$

Two important parameters are the characteristic impedance of free space, $\eta_{0}$, and the material's characteristic impedance, $\eta$ which are shown in (3.15) and (3.16), correspondingly.

$$
\begin{gathered}
\eta_{0}=\sqrt{\mu_{0} / \varepsilon_{0}} \\
\eta=\sqrt{\mu / \varepsilon}
\end{gathered}
$$

\subsection{Extended Debye Model}

As it was mentioned before, concrete and grout are both dispersive materials. Hence, their constitutive parameters $(\varepsilon, \mu, \sigma)$ vary with frequency and they will be analyzed using the Debye model [15]. This mathematical model is used to predict the interaction of electromagnetic EM waves with materials depending on the operational frequency. This modeling is crucial for solving problems related to electromagnetic transmission and propagation of communication signals.

According to the extended Debye model [6], the real $\varepsilon_{r}^{\prime}(\omega)$ and imaginary $\varepsilon_{r}^{\prime \prime}(\omega)$ parts of the complex permittivity of a material can be found as follows:

$$
\begin{gathered}
\varepsilon_{r}^{\prime}(\omega)=\varepsilon_{\infty}+\frac{\Delta \varepsilon}{1+\omega^{2} \tau^{2}} \\
\varepsilon_{r}^{\prime \prime}(\omega)=\frac{\omega \tau \Delta \varepsilon}{1+\omega^{2} \tau^{2}}+\frac{\sigma_{d c}}{\omega \varepsilon_{0}}=\frac{\sigma_{e f f}(\omega)}{\omega \varepsilon_{0}}
\end{gathered}
$$

where $\Delta \varepsilon$ is the difference between the values of the real part of relative permittivity at low frequency, $\varepsilon_{0}$ and at high frequency, $\varepsilon_{\infty} ; \tau$ is the relaxation time, $\omega$ is the angular frequency, and $\sigma_{d c}$ is the electrical conductivity of the material. 
The real part of the dielectric permittivity, $\varepsilon_{r}^{\prime}$, is related to the amount of electrical energy the material can store. The imaginary part, $\varepsilon_{r}^{\prime \prime}$, on the other hand, accounts for energy losses due to dielectric relaxation. In order to construct the Debye model of a material we needed the real measurements of the complex dielectric permittivity of the material. Therefore, the next section discusses relevant material characterization methods.

\subsection{Material Characterization Methods}

As it was explained in Section 3.2, in order to model the dependency of the complex permittivity of a material versus frequency, it is necessary to experimentally determine the dielectric permittivity at low frequency and at high frequency. Therefore, current measurements technologies were reviewed in order to determine a suitable one for our application. This section briefly focuses on two main methods for characterizing the properties of materials: resonant methods, and non-resonant methods. Non-resonant methods are mainly used for measuring electromagnetic properties over a frequency range while resonant methods are evaluated in specific frequency values.

Resonant methods are more accurate and sensitive than non-resonant methods. They are also more suitable for low loss samples. These methods rely on the use of resonator-perturbation method.

In non-resonant methods, the material properties are determined by calculating the material intrinsic impedance and the wave velocity in the material. When an electromagnetic wave travels from one medium to another, it results on a change on the material characteristic impedance and the wave velocity. Therefore, reflected and 
transmitted waves are present. Both can be determined by relating the characteristic impedances and wave velocity on both materials.

Non-resonant methods can be either (1) reflection methods or (2) transmission/reflection methods. Reflection methods calculate the material properties by measuring the reflection from the sample. Transmission/reflection methods, on the other hand, determine the material properties by analyzing both reflections and transmission though a sample. Both methods require a contact with the material. Then, both reflected and transmitted energy are calculated.

Reflection methods direct energy toward a sample of the material. The material properties are deduced by calculating the reflection coefficient between the material and an already established reference plane. Two types of reflections are calculated: openended reflection, and short-circuit reflection In both cases coaxial cables are used due to their properties of covering broad frequency bands.

In this work, the Open-Ended Reflection Method is used. This method required the following equipment (1) an engineered probe that contact the DUT, (2) a coaxial cable, and (3) a vector network analyzer (VNA) for measurements. A commercially engineered probe was placed in contact with the sample that was measured. Then, this probe was connected to the VNA via a coaxial cable as shown in Figure 3.1. The dimensions of the probe are shown in Figure 3.2. 


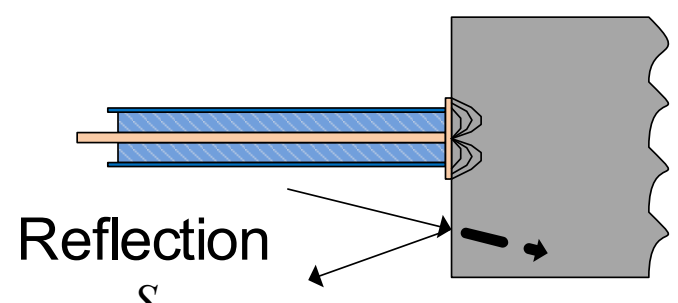

$S_{11}$

Figure 3.1 Reflection non-resonant method for complex dielectric permittivity measurements.

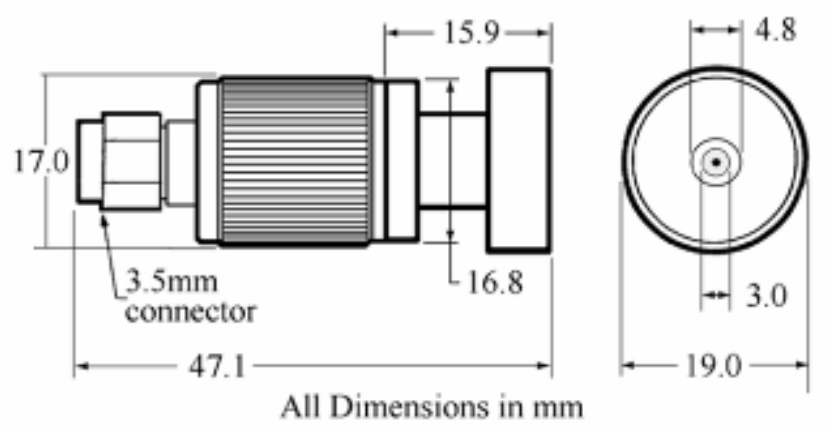

Figure 3.2 Dimensions of the used open-ended probe.

Basically, the VNA transmits an incident wave into the DUT via the coaxial cable and measures the reflected wave. Vector network analyzers (VNAs) are used to measure the scattering parameters $\quad, \quad, \quad$, and . A network analyzer possesses a source, signal separation devices, and detectors. The scattering parameters are obtained by combining two forward traveling waves and two reverse traveling waves. Detectors measure the four corresponding waves, and the signal separation devices make sure the four waves are measured, separately. 
Finally, the network analyzer detects the change in the impedance loading due to the introduction of the sample or device under testing (DUT). This impedance discontinuity caused by the sample loading allows deriving the properties of the material. Impedances on each side are different. Therefore, a reflection will happen if an electromagnetic wave propagates through the interface.

For this work, a commercial open-ended probe, Dielectric Probe HP85070B [16] from Hewlett Packard with dimensions shown in Figure 3.2, was used. Then, an HP software, which extracts the material properties from the measurements, was used. This software can use either the Cole-Cole or the Debye material models and provide the following constants: (1) the low frequency dielectric constant, $\varepsilon_{o}$, (2) the high frequency dielectric constant, $\varepsilon_{\infty},(3)$ the relaxation width, $\alpha$, and (4) the relaxation time constant, $\tau$. These parameters lead to the calculation of the complex dielectric permittivity of a material.

\subsection{Measurements of Material Properties}

Before conducting any measurement, the equipment must be properly calibrated. Calibration corrects losses introduced by the cable, and the equipment. Calibration for our method was performed in three steps: (1) the probe was open, (2) the probe was shorted, and (3) the probe was immersed in water. The probe calibration with deionized water can be observed in Figure 3.3. After calibration was finished, deionized water was measured again. Then, in order to assess the accuracy of the measurements, measured results were compared with known Debye model results [17] as shown in Figure 3.5, and 
Figure 3.6. Figure 3.5, and Figure 3.6, show the real and imaginary parts of the dielectric permittivity of deionized water, respectively.

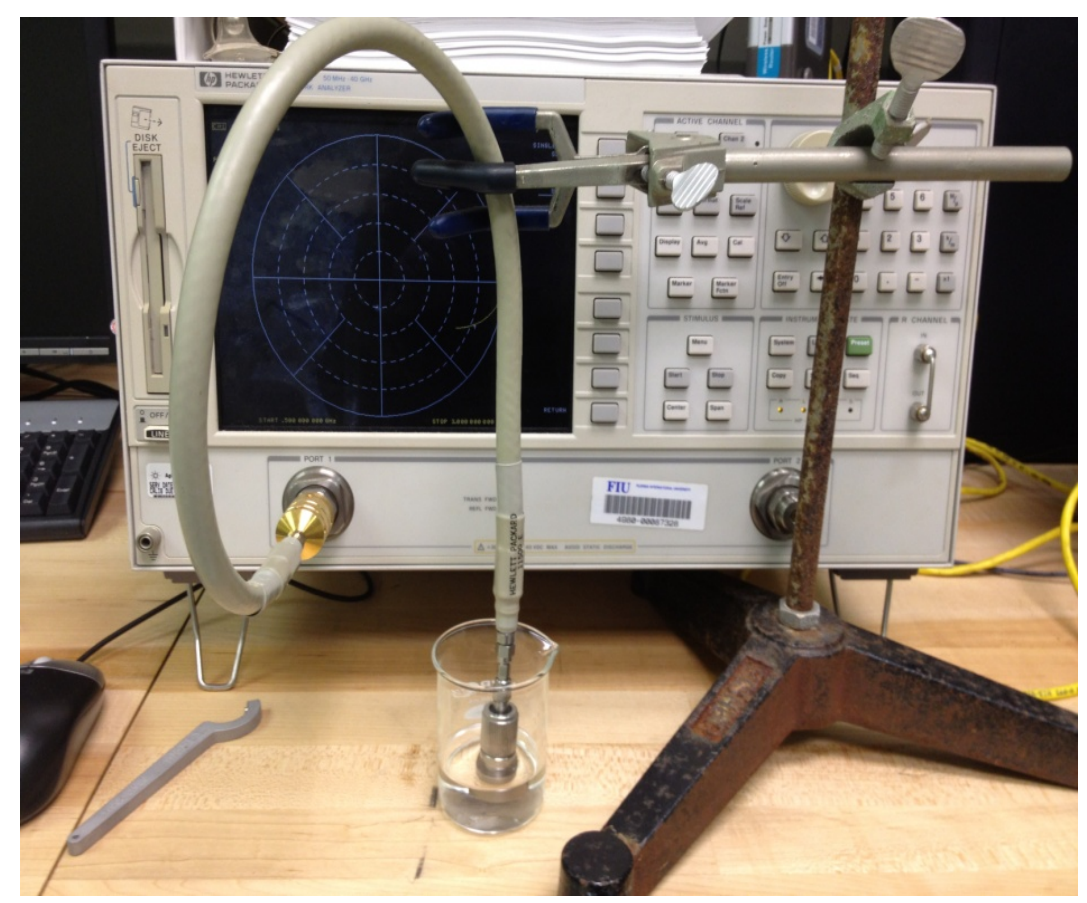

Figure 3.3 Calibration and measurement of dielectric properties of deionized water.

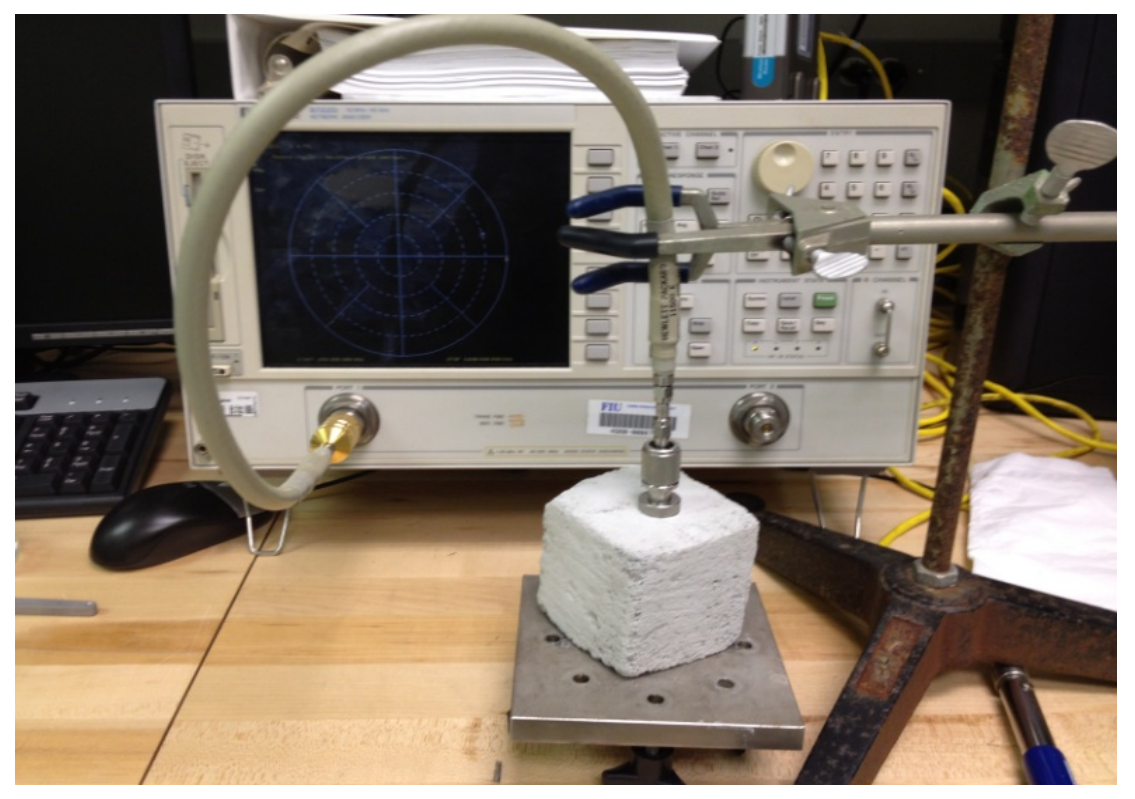

Figure 3.4 Measuring grout with an open-ended probe. 


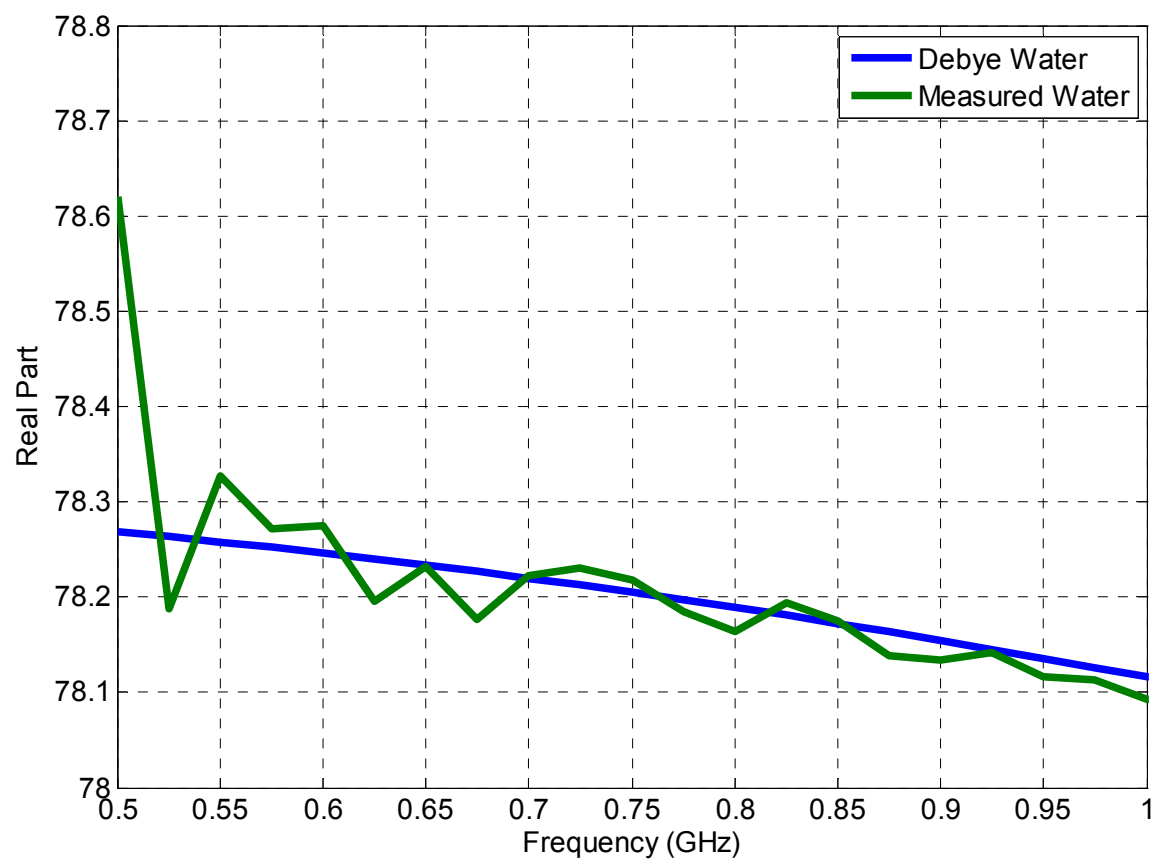

Figure 3.5 Real part of the complex dielectric permitivity of water.

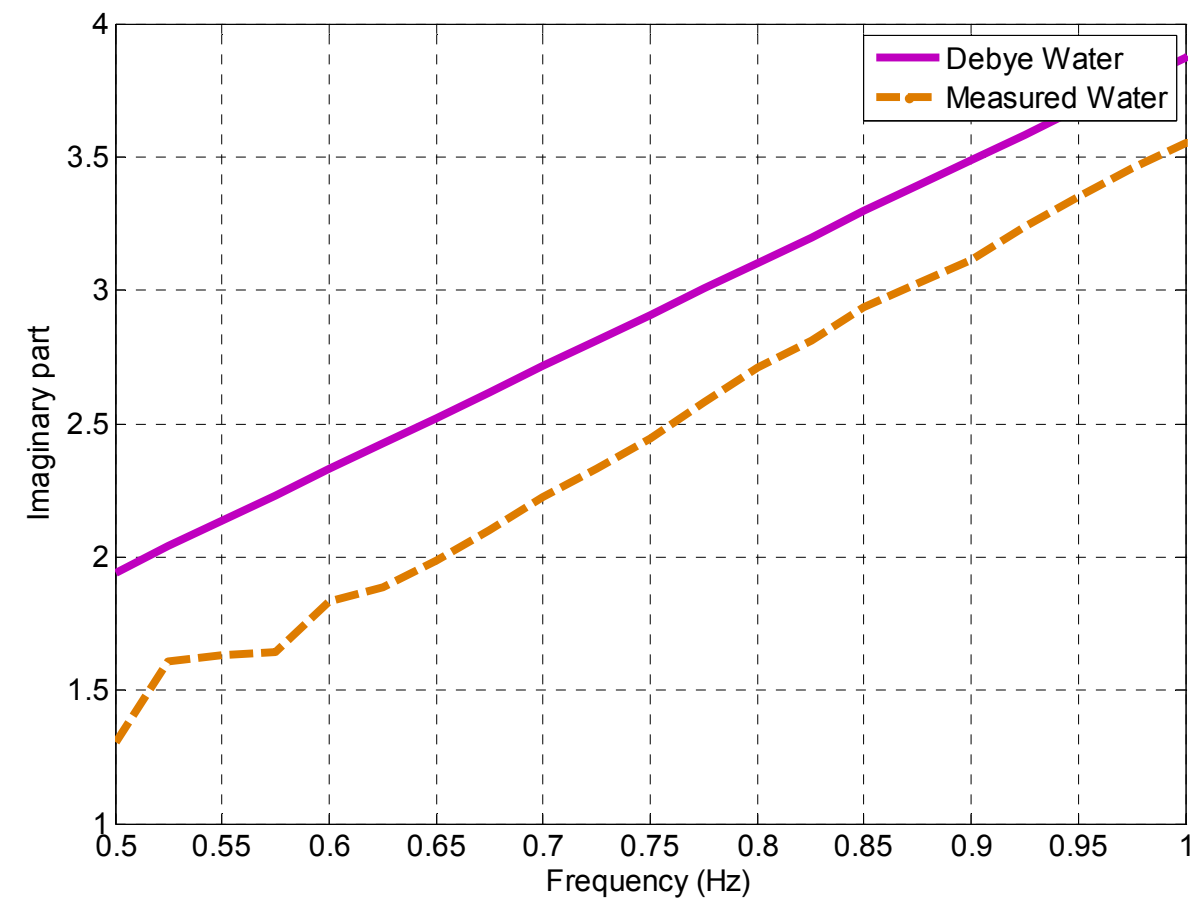

Figure 3.6 Imaginary part of the complex dielectric permitivity of water. 
The relative error between the measured water and the known Debye model was approximately $2 \%$. This error is considered acceptable for our application. Then, the next measurement was performed on the grout as shown in Figure 3.4. Figure 3.7 shows the complex dielectric permittivity of concrete from $0.1 \mathrm{GHz}$ to $1 \mathrm{GHz}$. For the grout, measurements were performed covering the range from $0.1 \mathrm{GHz}$ to $1 \mathrm{GHz}$ as well. Figure 3.8 shows the measured real and imaginary part of the dielectric permittivity of the grout.

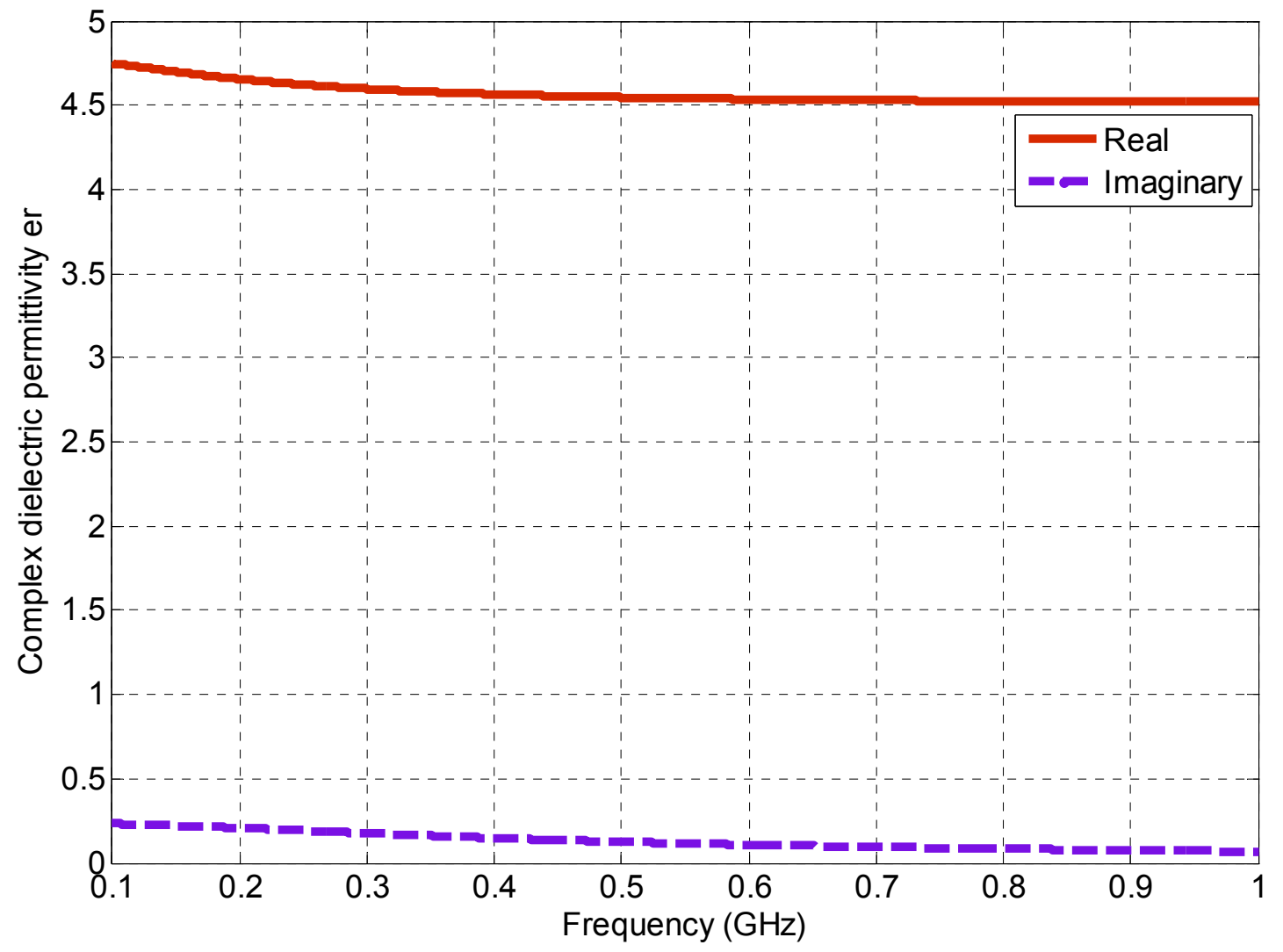

Figure 3.7 Complex dielectric permittivity of concrete. 


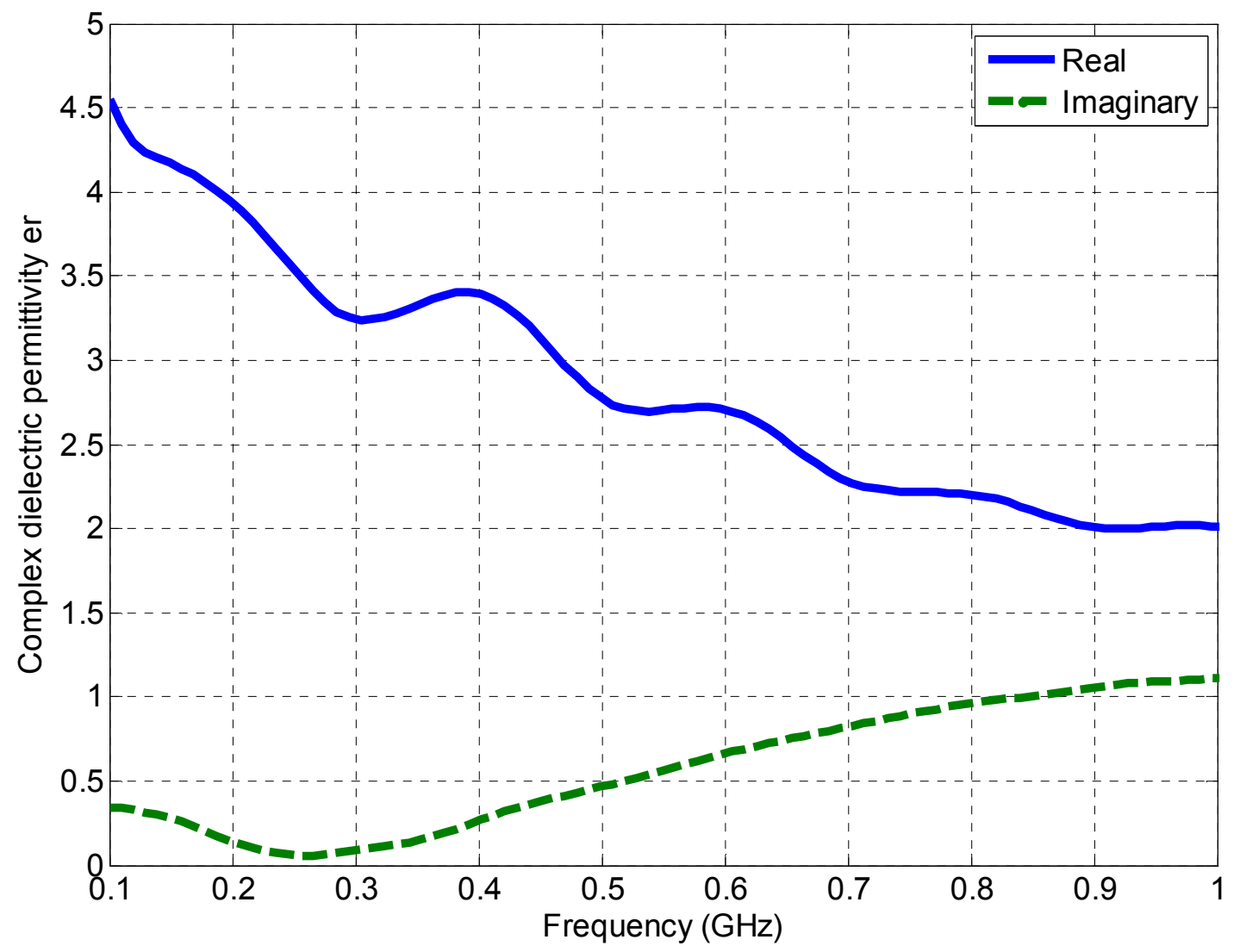

Figure 3.8 Complex dielectric permittivity of grout.

It can be observed in both Figure 3.7 and Figure 3.8, that the real part of the dielectric permittivity has larger values at lower frequencies than the ones at higher frequencies. This means that less electrical energy can be stored at higher frequencies. Similarly, higher values of the imaginary part of the complex dielectric permittivity translate into higher losses due to dielectric relaxation. Therefore, it should be expected that a uniform plane wave will suffer more losses at higher frequencies than at lower frequencies.

Also, by comparing the complex dielectric permittivity of the two materials, concrete and grout, it can be observed that concrete exhibits larger values of the real part 
of the dielectric permittivity than grout. Additionally, the imaginary part for grout shows higher imaginary part of the dielectric permittivity compared to concrete. Therefore, EM waves suffer fewer losses inside concrete than grout. Chapter 4 validates this expectation. 


\section{FORMULATION OF A UNIFORM PLANE WAVE PENETRATION INSIDE CONCRETE AND GROUT}

In the previous chapter, the constitutive parameters of grout were measured from $0.1 \mathrm{GHz}$ to $1 \mathrm{GHz}$. In this research, the communication link, which is established between two devices that were positioned in different materials, one in air, and one in a lossy material (i.e., grout), was examined. Since the communication signal travels from one material into another, reflection and transmission occur at the boundary between the two materials. This chapter determined both the transmission and propagation coefficients in terms of the constitutive parameters, angle of incidence, and wave polarization. Finally, these coefficients were used to determine transmission and propagation losses, $A_{t}$, and, $A_{p}$, in order to calculate the total losses that a uniform plane wave suffers while traveling from air into a lossy material, such as concrete and/or grout.

Transmission and propagation losses inside a material were calculated versus frequency for both concrete and grout. These results can assist the design of embedded wireless sensors systems in such materials. Previous work has examined the modeling of RF propagation inside concrete [6]. This same approach can be used for other building materials, such as grout. In this chapter, the transmission and propagation losses of a uniform plane wave were calculated for novel mixtures of grouts used for decommissioning of nuclear plants. In the case of concrete, different humidity contents have been analyzed and the material properties have been modeled using the Debye model [15], [18], [19]. Also, the transmission losses and propagation losses have been calculated, and an optimum frequency range was determined were losses are minimum [6]. 


\subsection{Plane wave model}

For analytical purposes the total power loss or total attenuation, $A_{\text {TOTAL }}$, was evaluated for a plane wave penetrating from air into a lossy medium, such as concrete and grout. First, the boundary conditions were established between two materials with different constitutive parameters as shown in Figure 4.1. For the given boundary conditions, there are two types of attenuation, transmission and propagation, which if they are added they provide the total loss, $A_{\text {TотAL }}$. Transmission losses, $A_{t}$, occur when the uniform plane wave passes from one medium (air) to another (concrete or grout). After the wave has penetrated into the medium, it continues propagating inside it and attenuates proportionally to the distance of propagation. This attenuation is known as propagation loss, $A_{p}$.
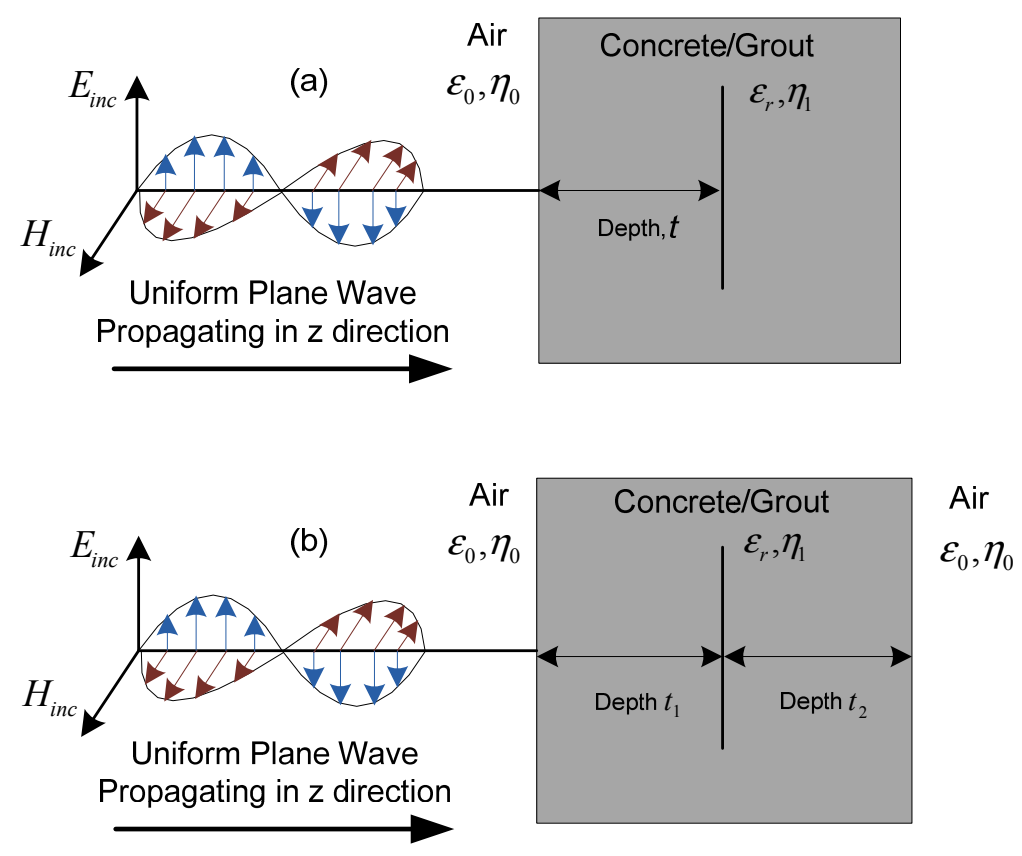

Figure 4.1 Plane wave model for normal incidence in (a) half-space, and (b) finite slab approach. 
The propagation of a uniform plane wave is influenced by the angle of incidence. Therefore, it should be analyzed in two different scenarios (a) normal incidence, and (b) oblique incidence.

\subsection{Normal Incidence}

In previous work of concrete [6], two cases have been analyzed. The first case considered the infinite or half-space [see Figure 4.1]. The second case when the material is considered finite known as a slab case [see Figure 4.1]. Here we examined the halfspace first. First, the incidence power $P_{\text {inc }}$ is determined as described in (4.1) [20]:

$$
\begin{gathered}
P_{\text {inc }}=\operatorname{Re}\left(\left|E_{\text {inc }}\right|^{2} / 2 \eta_{0}\right) \\
\eta_{0}=\sqrt{\mu_{0} / \varepsilon_{0}}
\end{gathered}
$$

where:

$E_{\text {inc }}$ is the incident electric field,

$\eta_{0}$ is the intrinsic impedance of air,

$\mu_{0}$ is the permeability of air in free space, and

$\varepsilon_{0}$ is the dielectric permittivity of air.

The transmitted power $P_{t}$ is determined as follows:

$$
\begin{gathered}
P_{t}=\operatorname{Re}\left(\left|E_{t}\right|^{2} / 2 \eta_{1}^{*}\right)=\operatorname{Re}\left(\left|E_{t}\right|^{2} / 2 \eta_{1}^{*}\right) \cdot|T|^{2} \cdot e^{-2 \alpha d} \\
\eta_{1}=\sqrt{\mu_{1} / \varepsilon_{0} \varepsilon_{r}} \\
T=E_{t} / E_{\text {inc }}=2 \eta_{1} / \eta_{1}+\eta_{0}
\end{gathered}
$$

where $E_{t}$ is the transmitted electric field,

$\eta_{1}$ is the intrinsic impedance of grout, or concrete,

$T$ is the transmission coefficient in half-space, 
$\mu_{1}$ is the permeability of the grout, or concrete, and $\varepsilon_{r}=\varepsilon_{r}^{\prime}-j \varepsilon_{r}^{\prime \prime}$ is the relative complex dielectric permittivity of concrete or grout.

After establishing these parameters the calculation for the transmission, propagation, and total losses, $A_{t}, A_{p}$ and, $A_{\text {total }}$ respectively are given by:

$$
\begin{gathered}
A_{t}=10 \cdot \log _{10}\left(|T|^{2} \cdot \operatorname{Re}\left(\eta_{0} / \eta_{1}^{*}\right)\right) \\
A_{p}=10 \cdot \log _{10}\left(e^{-2 \alpha t}\right) \\
A_{\text {total }}=A_{t}+A_{p}=10 \cdot \log _{10}\left(P_{t} / P_{\text {inc }}\right)
\end{gathered}
$$

where $t$ is the distance that the wave has propagated inside concrete or grout. The attenuation, $\alpha$, is calculated by (3.13) shown in the previous chapter.

Next, the slab case is examined. This case is similar to the half-space approach but it considers a finite thickness material, i.e., slab. For such a slab, the total losses $A_{\text {total }}$ are given by (4.9) [6]:

$$
A_{\text {total }}=10 * \log _{10}\left(\left|T_{\text {slab }}\right|^{2} * \operatorname{Re}\left(\frac{\eta_{0}}{\eta_{1}^{*}}\right)\right)
$$

where:

$$
\begin{gathered}
T_{\text {slab }}=|T| \sqrt{\frac{e^{-2 \alpha_{1} t_{1}}-2|\Gamma| e^{-2 \alpha_{1} t_{s}} \cos \left(2 \beta_{1} t_{2}\right)+|\Gamma|^{2} e^{-2 \alpha_{1} t_{s}} e^{-2 \alpha_{1} t_{2}}}{1-2|\Gamma|^{2} e^{-2 \alpha_{1} t_{s}} \cos \left(2 \beta_{1} t_{s}\right)+|\Gamma|^{4} e^{-4 \alpha_{1} t_{s}}}} \\
\Gamma=\frac{E_{r}}{E_{\text {inc }}}=\frac{\eta_{1}-\eta_{0}}{\eta_{1}+\eta_{0}} \\
t_{s}=t_{1}+t_{2}
\end{gathered}
$$

where $\alpha_{1}$ is the attenuation constant, and in $\beta_{1}$ is the phase constant inside concrete or grout. It should be noted that when $t_{2}$ infinite, equation (4.9) becomes equal to equation (4.6). 


\subsection{Oblique Incidence}

Here, the case of oblique incidence was examined as presented in [6]. It is expected that oblique incidence will not change the propagation loss, and it will only affect the transmission loss, $A_{t}$. For easier analysis, we examined separately the perpendicular and parallel polarizations of the electric field. Figure 4.2 shows the plane wave model for oblique.

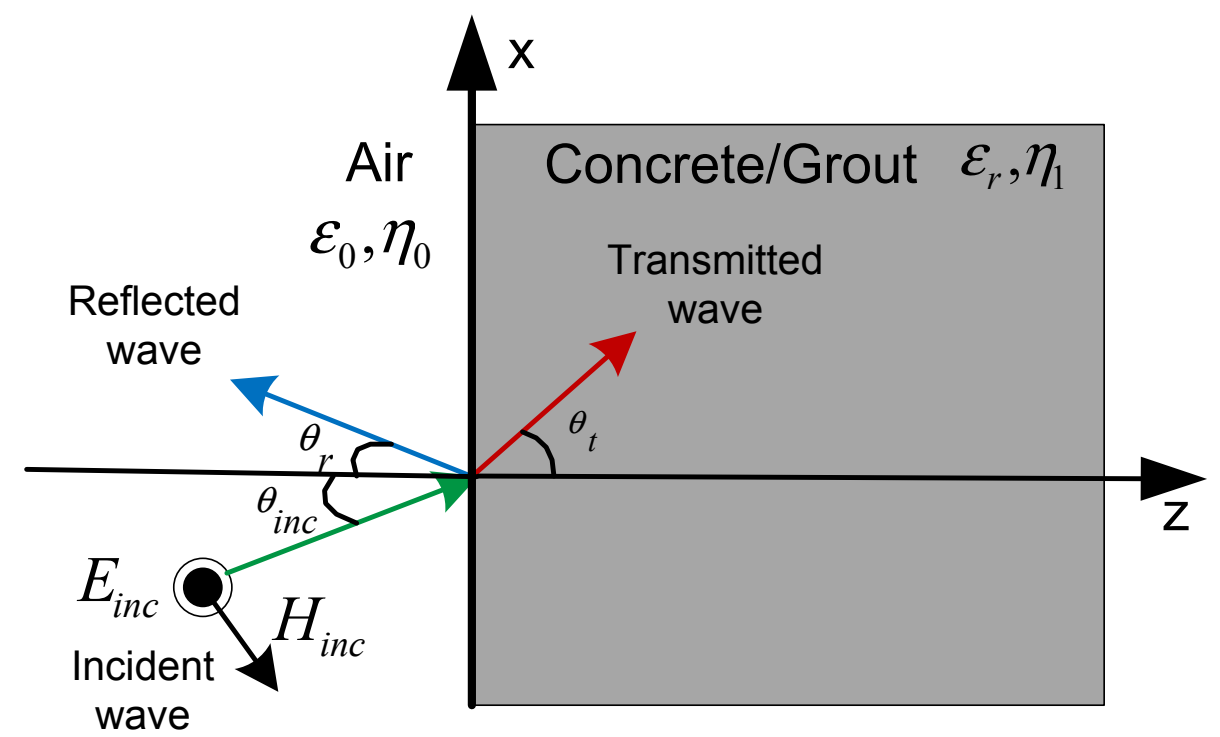

Figure 4.2 Plane wave model for oblique incidence.

The transmission coefficients for parallel and perpendicular polarization are written as [6]: 


$$
\begin{aligned}
& T_{||}=\frac{2 \eta_{1} \cos \theta_{i n c}}{\eta_{0} \cos \theta_{i n c}+\eta_{1} \cos \theta_{t}} \\
& T_{\perp}=\frac{2 \eta_{1} \cos \theta_{i n c}}{\eta_{1} \cos \theta_{i n c}+\eta_{0} \cos \theta_{t}}
\end{aligned}
$$

where $\theta_{\text {inc }}$ is the angle of incidence, and $\theta_{t}$ is the angle of transmission. Assuming that material is non-magnetic $\left(\mu_{0}=\mu_{1}=1\right)$, and the following equation can be derived for $\theta_{t}$ :

$$
\cos \theta_{t}=\sqrt{1-\sin ^{2} \theta_{i n c} / \varepsilon_{r}}
$$

Similarly, to the normal incidence, the propagation and total losses can be calculated using (4.7), and (4.8). The next section derives the results for concrete and grout.

\subsection{Transmission and Propagation losses inside concrete and grout}

Initially, the losses inside concrete were calculated and our results were compared to the ones of [6] for validation purposes. Then, our code was applied to calculations related to grout. The transmission losses for normal incidence are shown for concrete in Figure 4.3. Moreover, for oblique incidence, the transmission losses, $A_{t(||)}$, and, $A_{t(\lrcorner)}$, for parallel and perpendicular polarization, respectively, are shown in Figure 4.4. In the oblique incidence case, the angle of incidence, $\theta_{i n c}$, was varied from $0^{\circ}$ to $90^{\circ}$ with intervals of $5^{\circ}$. It is observed that the transmission losses for normal incidence are larger than the ones for oblique incidence.

The propagation losses, $A_{p}$, on the other hand, depend directly on the distance, $t$, that the EM wave has to travels inside concrete or grout. Therefore, propagation losses increase proportionally with the distance. Propagation losses, $A_{p}$, were calculated for 
depths of, $t, 10 \mathrm{~cm}, 20 \mathrm{~cm}, 30 \mathrm{~cm}$ and $40 \mathrm{~cm}$ inside concrete and grout. Since the propagation loss do not depend on the wave angle of incidence, and therefore, they are the same for both normal and oblique incidence. The results of the propagation losses for concrete are shown in Figure 4.5.

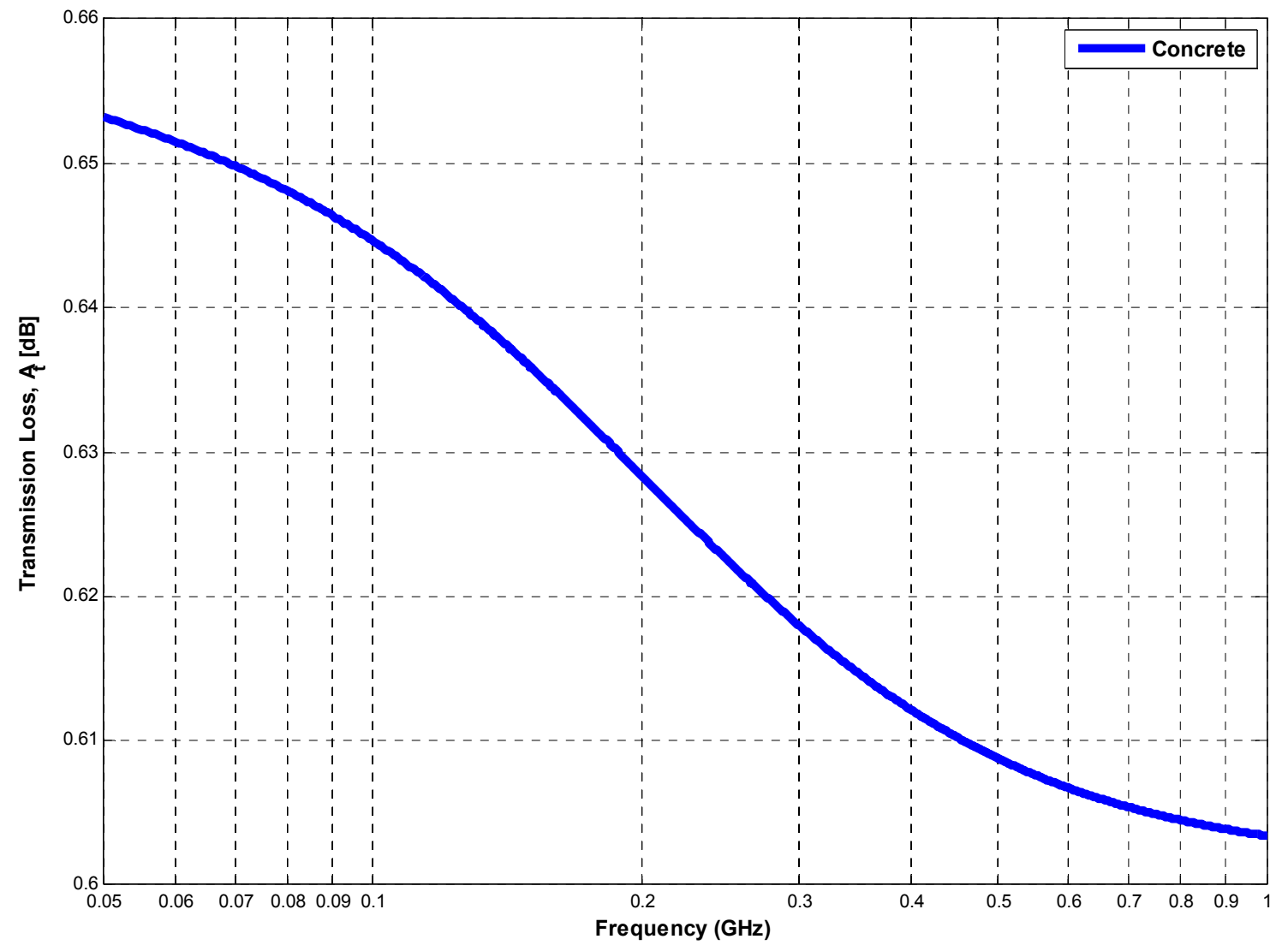

Figure 4.3 Transmission loss, $A_{t}$, for concrete in normal incidence. 


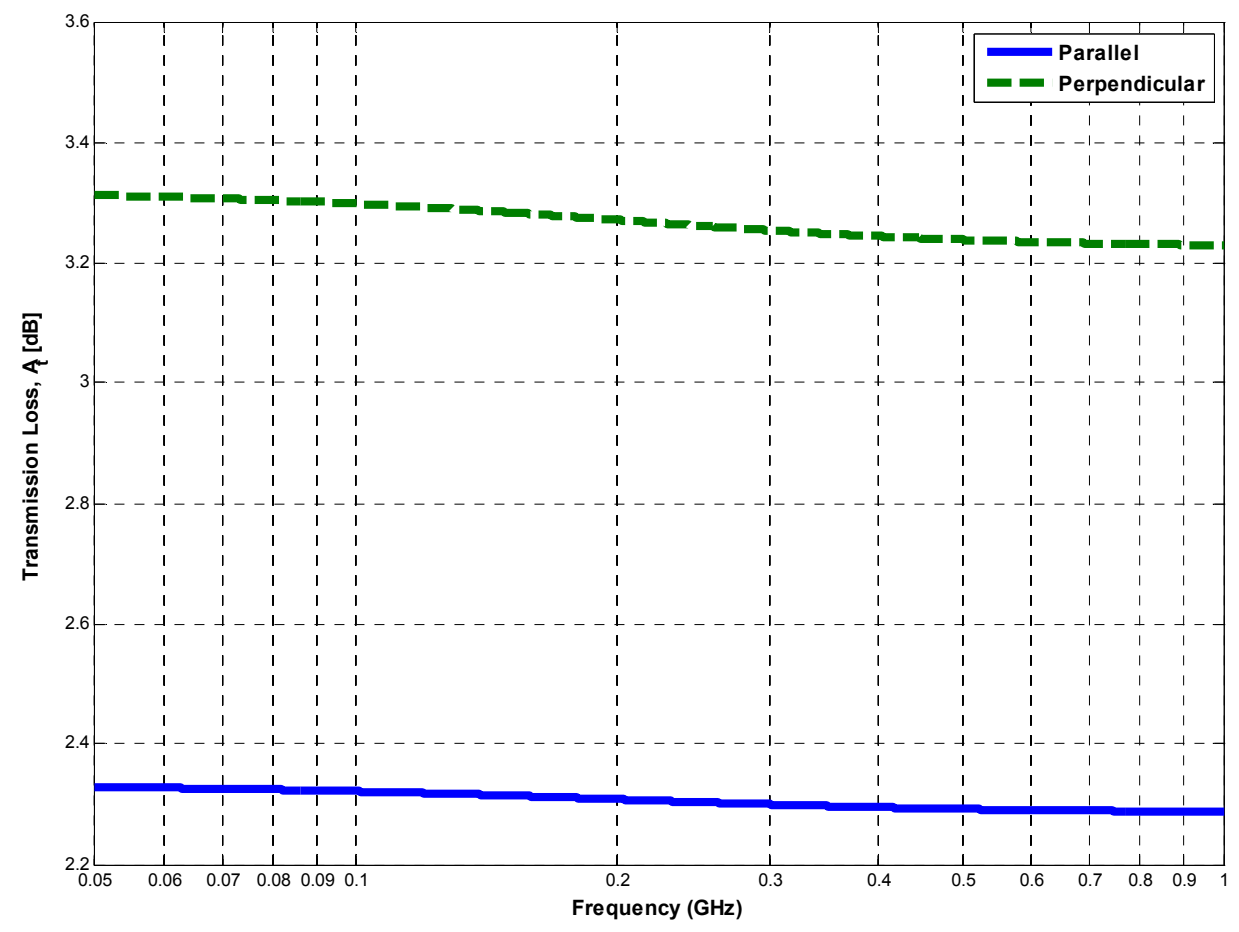

Figure 4.4 Transmission loss, $A_{t}$, for concrete in oblique incidence.

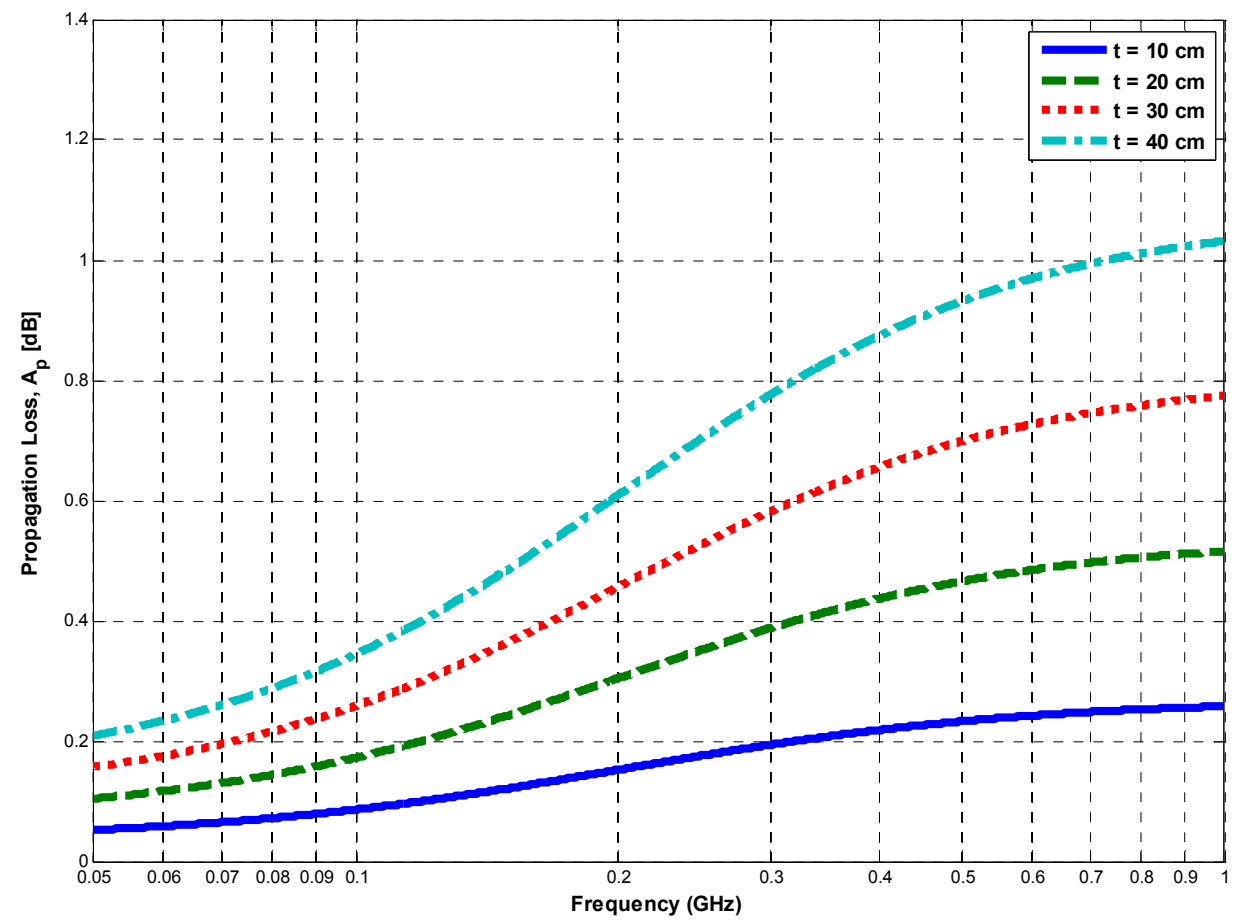

Figure 4.5 Propagation loss, $A_{p}$, for concrete for different depths, $t$. 
Then, the total loss, $A_{\text {TOTAL}}$, for both the half-space and slab cases for normal incidence in concrete are illustrated in Figure 4.6, and Figure 4.7. It can be observed that the total losses are smaller for the half-space case than for the slab case.

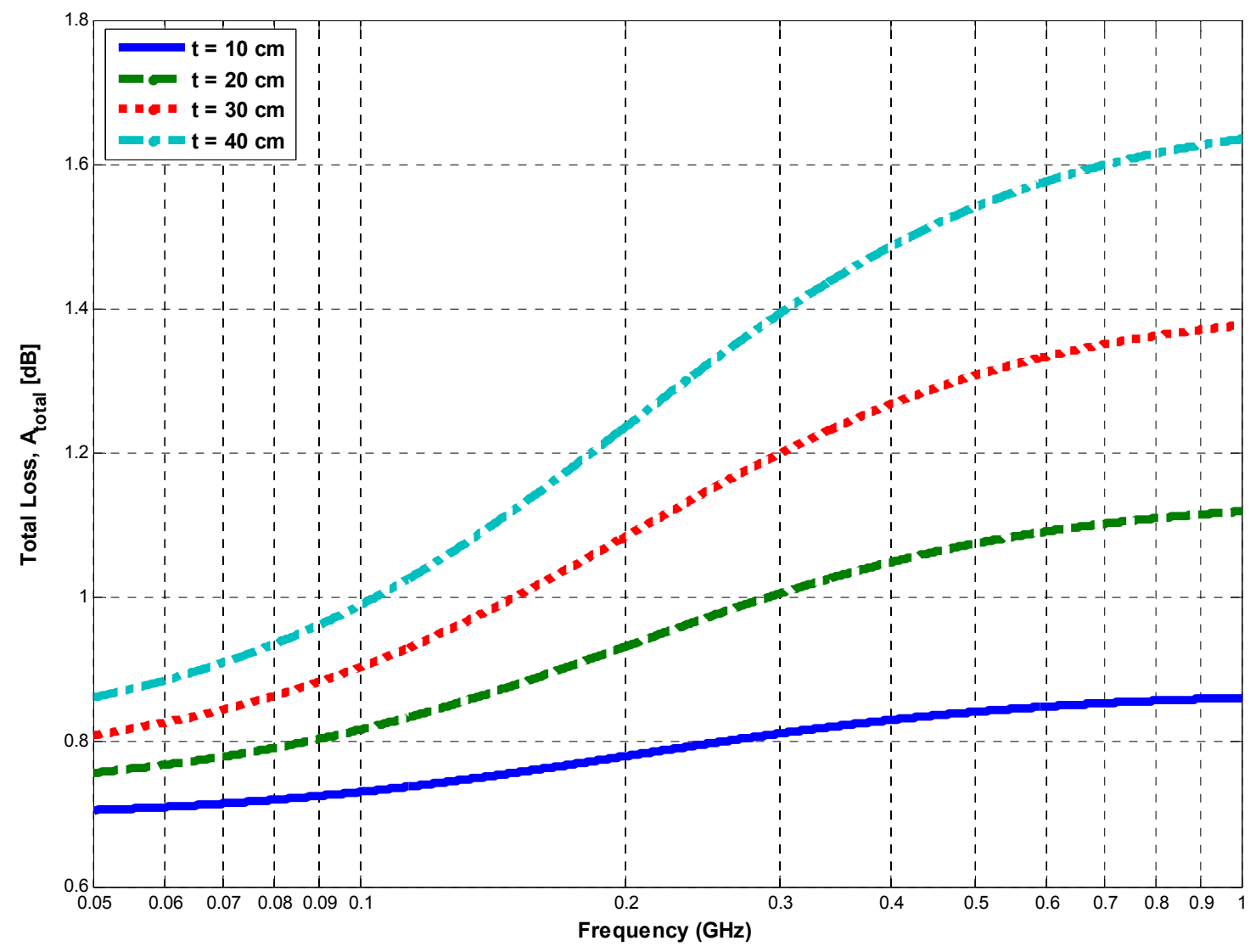

Figure 4.6 Total loss, $A_{\text {TOTAL }}$ for different depths, $t$, inside half-space concrete for normal incidence. 


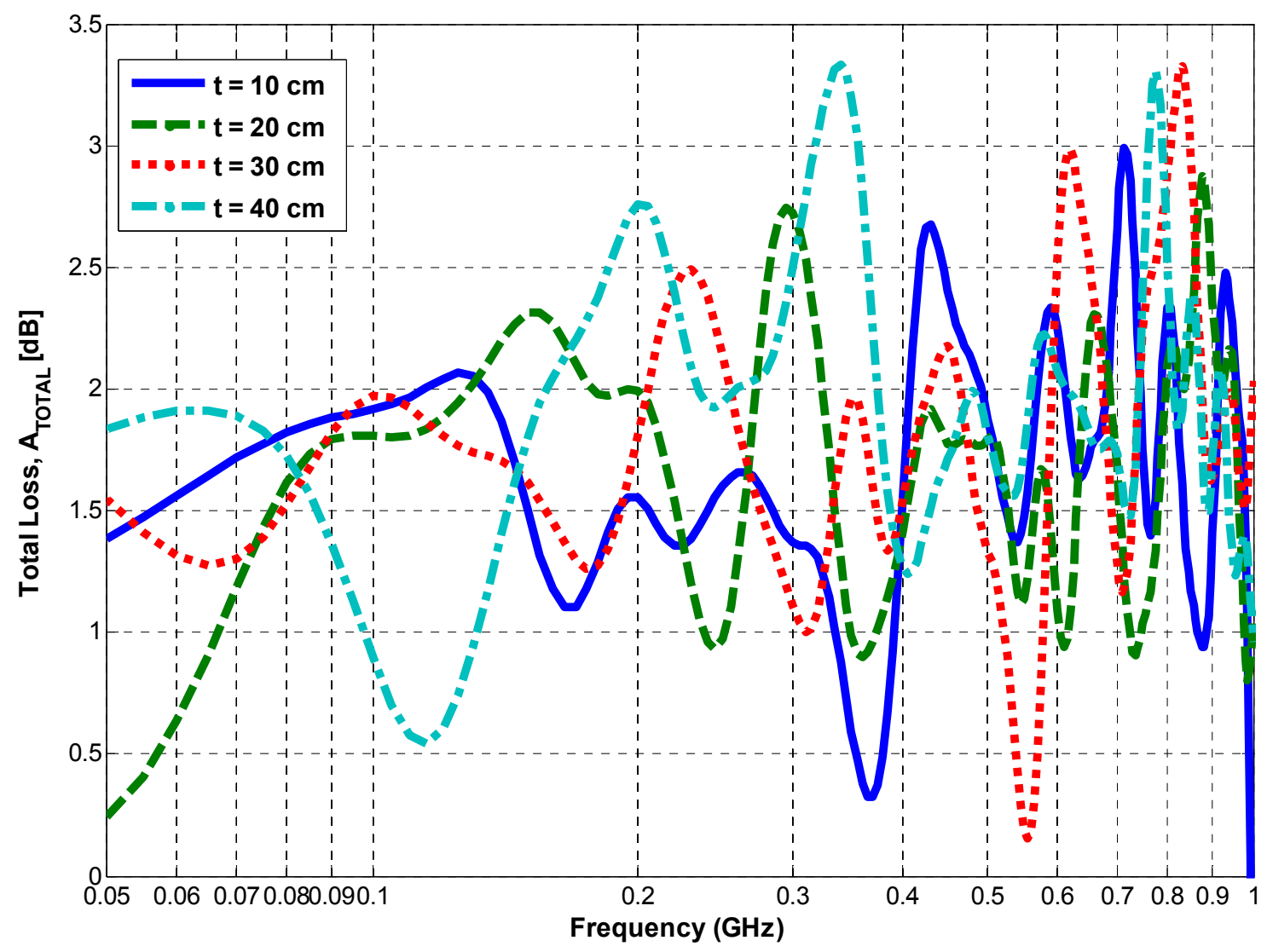

Figure 4.7 Total loss, $A_{\text {TOTAL }}$ for concrete slabs with different depths, $t$, for normal incidence.

Finally, the total losses, $A_{\text {TотAL, }}$ are plotted for both the parallel and perpendicular cases for oblique incidence on concrete. In Figure 4.8, and Figure 4.9 it can be seen that the parallel polarization case exhibits less losses than the losses of the perpendicular case. 


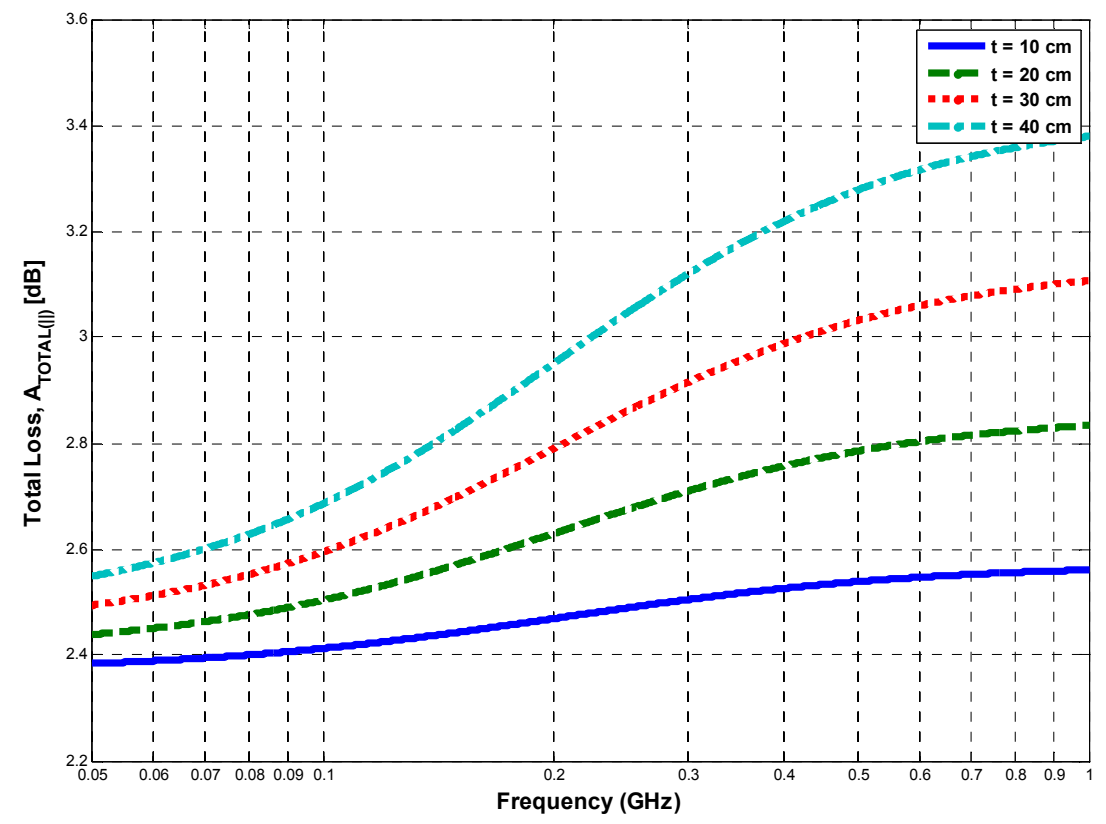

Figure 4.8 Total loss, $A_{\text {TOTAL (I) }}$ for different depths, $t$, inside concrete in half-space case at oblique incidence.

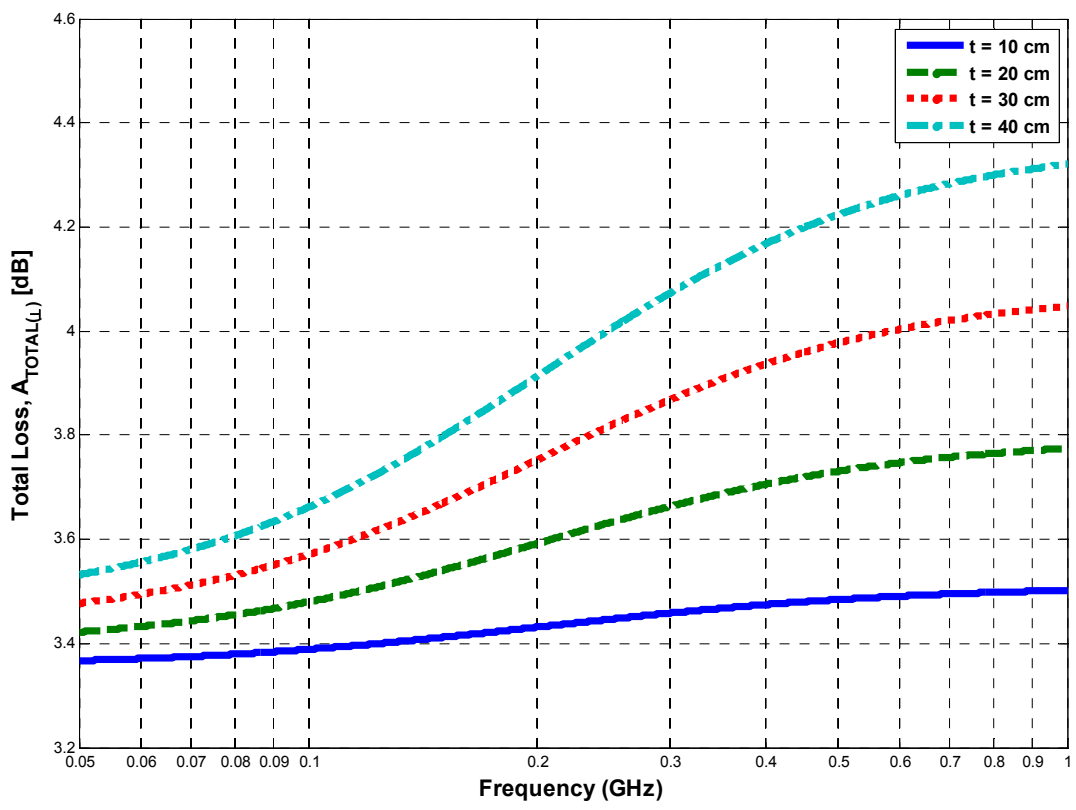

Figure 4.9 Total loss, $A_{\text {TOTAL( }\lrcorner)}$ for different depths, $t$, inside concrete in half-space case at oblique incidence. 
The second part of this section presents the same analysis for grout. The transmission losses, $A_{t}$, are depicted in Figure 4.10, and Figure 4.11.

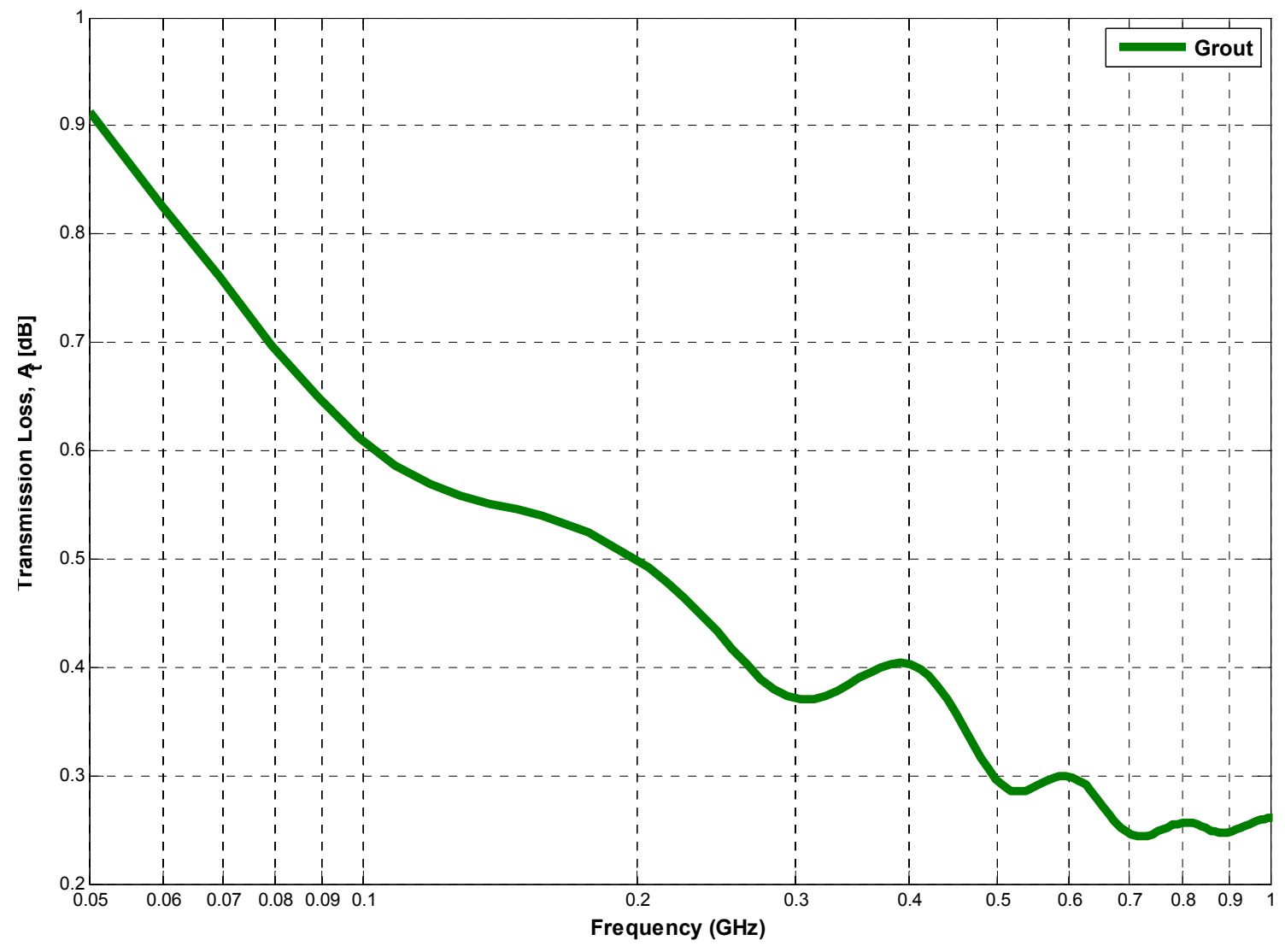

Figure 4.10 Transmission loss, $A_{t}$ of grout in normal incidence. 


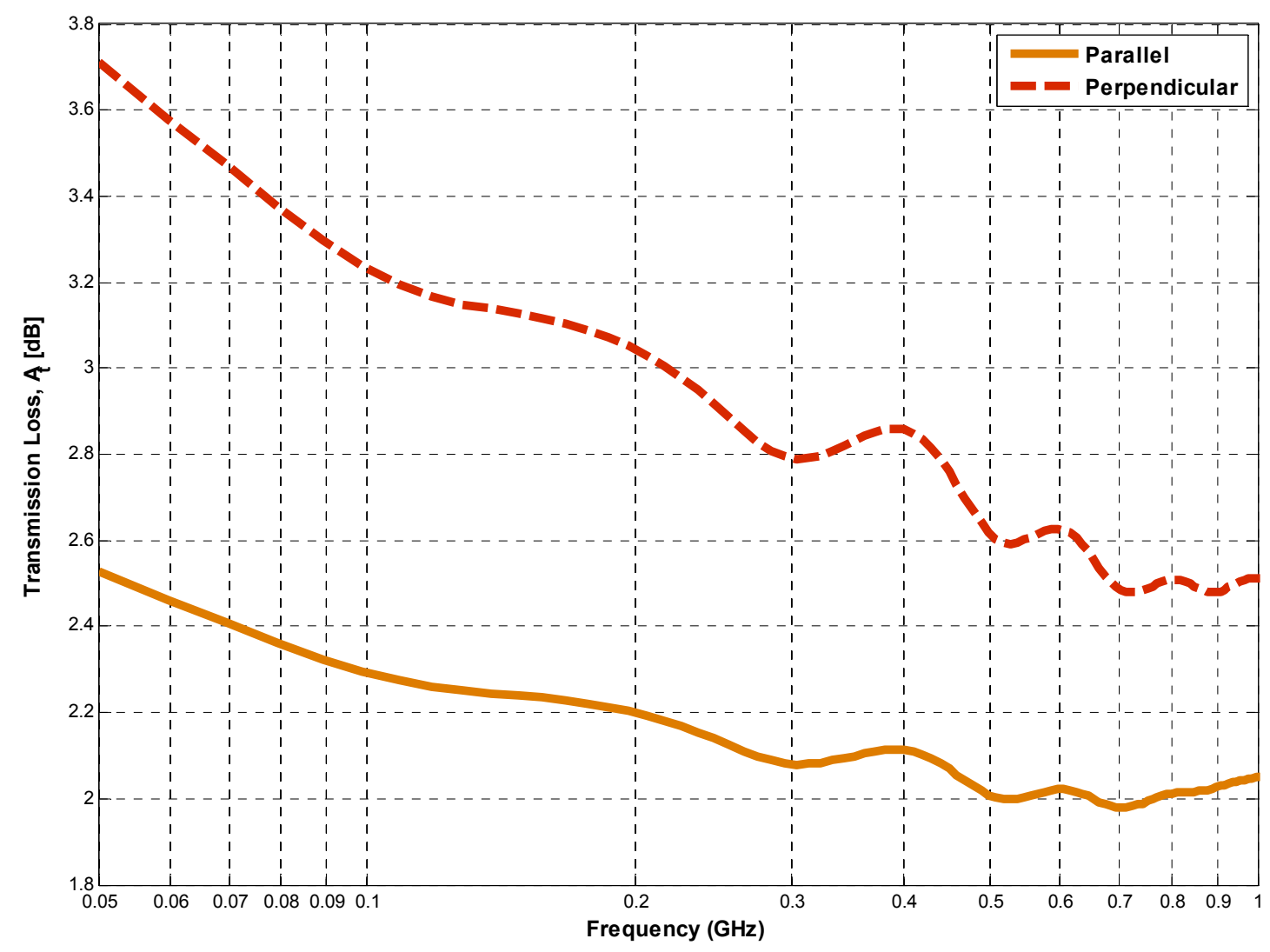

Figure 4.11 Transmission loss, $A_{t}$ of grout in oblique incidence.

The propagation losses, $A_{p}$, for grout were modeled for different depths, $t$, in both normal and oblique incidence as shown in Figure 4.12. Then, for normal incidence, the total losses were calculated and shown for half-space and slab cases in Figure 4.13, and Figure 4.14, respectively. For the slab case, oscillating behavior is observed due to the signal reflecting inside the material. An increment in the losses at lower frequency can be noticed as well. Finally, the last two cases were the total loss, $A_{\text {TOTAL }}$, were calculated for oblique incidence are presented in Figure 4.15, and Figure 4.16. Similarly to concrete, the losses increment for oblique incidence. 


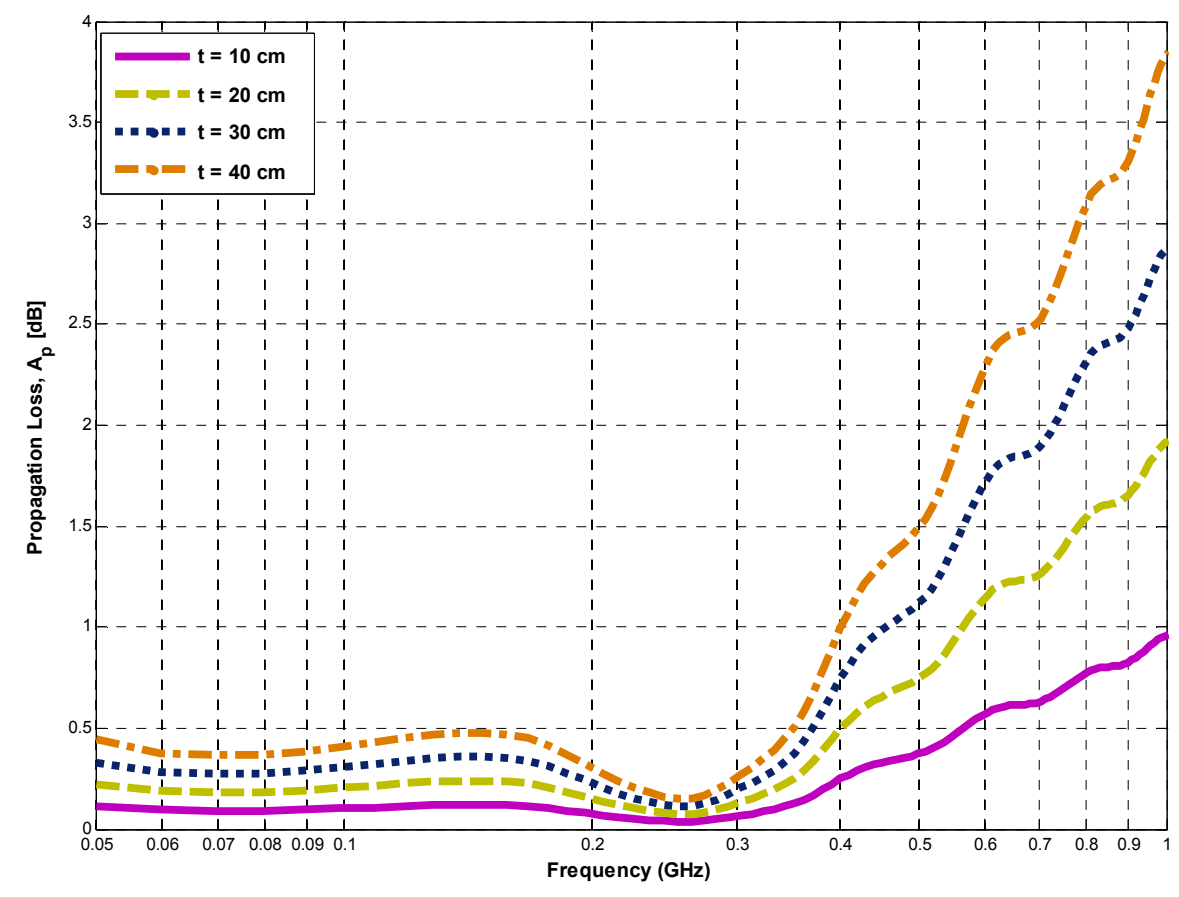

Figure 4.12 Propagation loss, $A_{p}$ for grout with different depths, $t$.

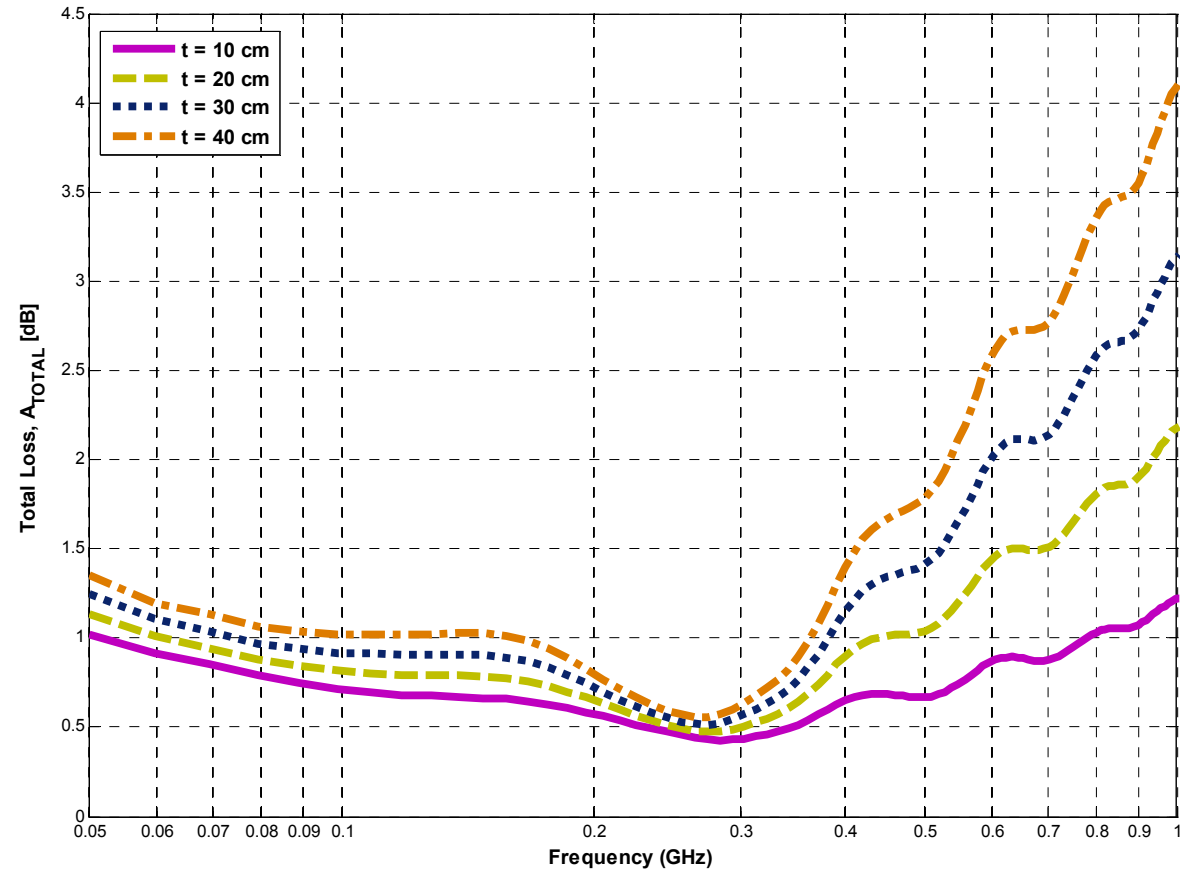

Figure 4.13 Total loss, $A_{\text {ТотАL }}$ for grout with different depths, $t$, in half space at normal incidence. 


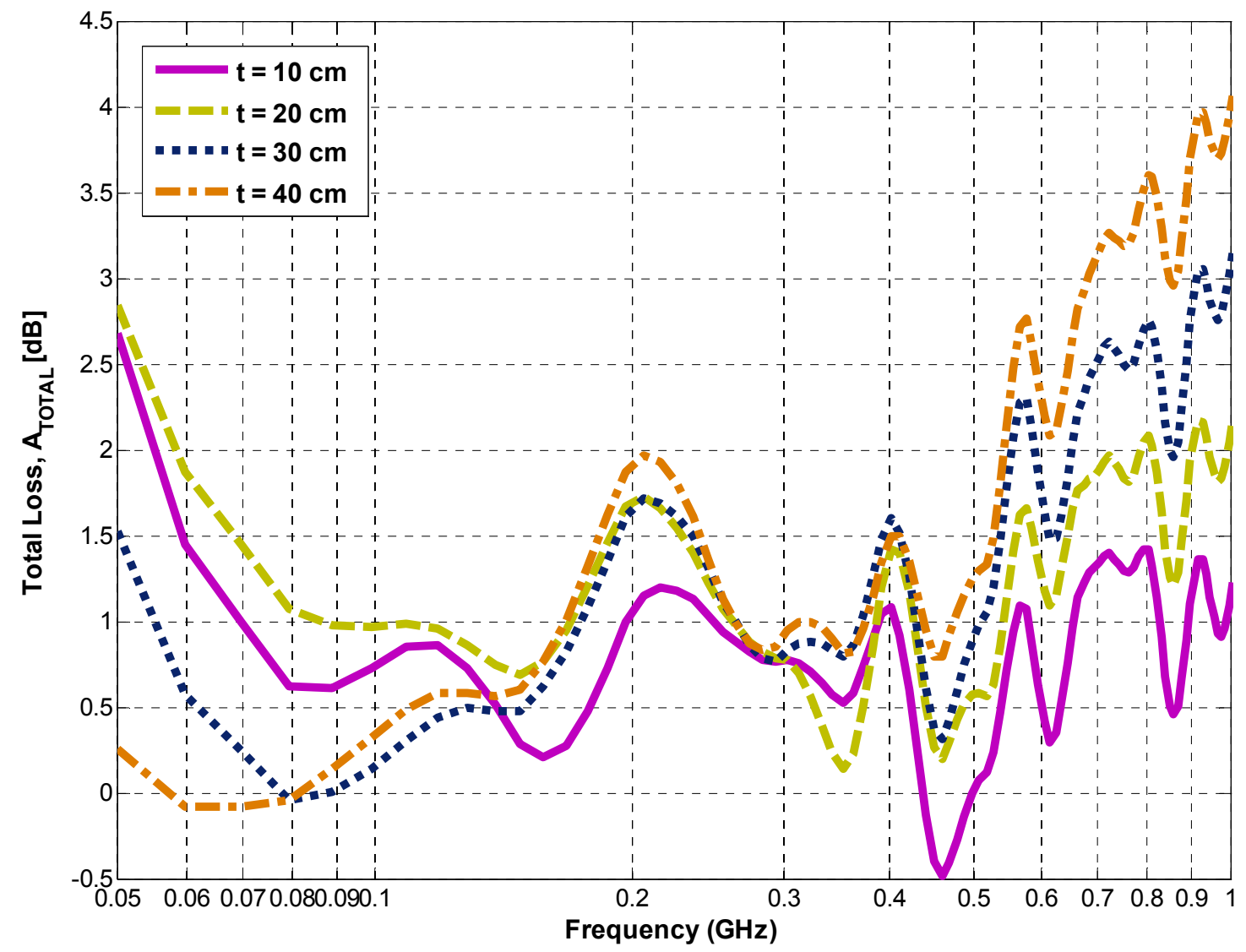

Figure 4.14 Total loss, $A_{\text {TотAL }}$ for grout with different depths, $t$, in slab approach at normal incidence. 


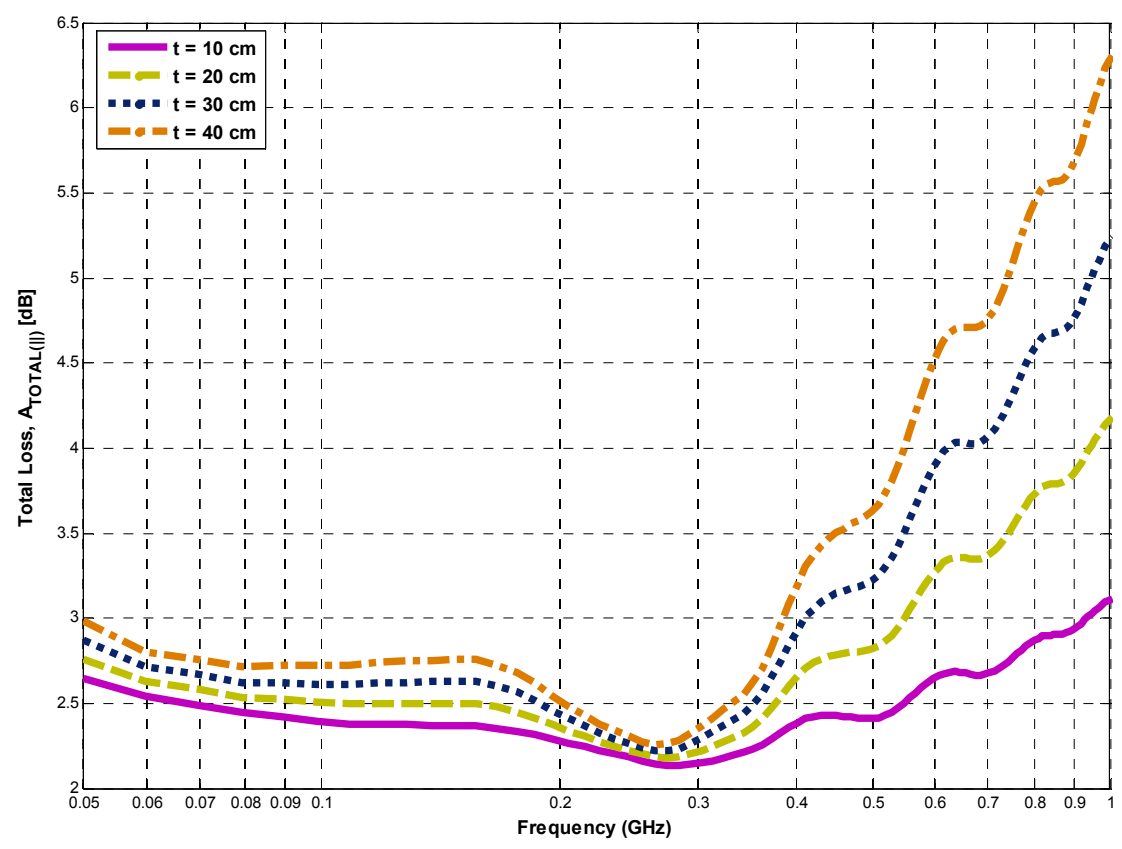

Figure 4.15 Total loss, $A_{\text {TOTAL(II) }}$ of grout with different depths, $t$, in half-space at oblique incidence.

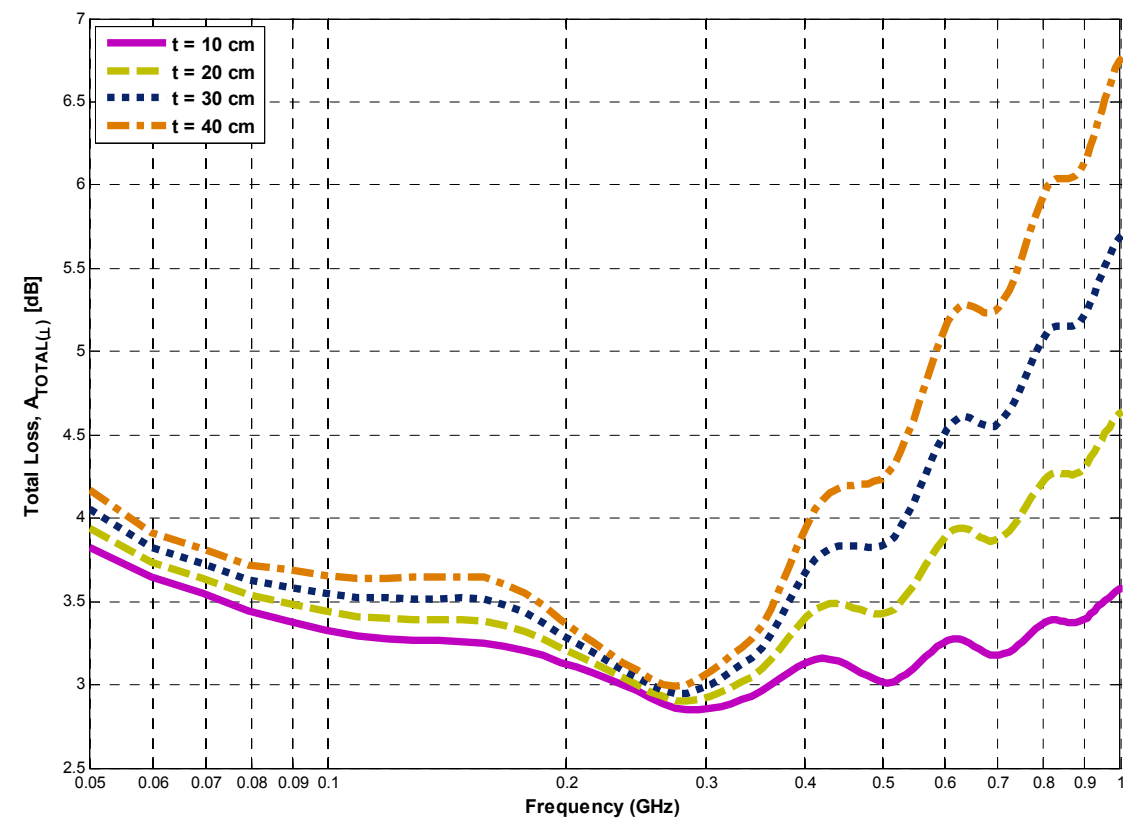

Figure 4.16 Total loss, $A_{\text {TOTAL( }\lrcorner)}$ of grout with different depths, $t$, in half-space at oblique incidence. 
It can be seen from these results that, an optimum frequency range occurs around $300 \mathrm{MHz}$ where the losses are minimal. Specifically, for the frequency of interest of $f=$ $915 \mathrm{MHz}$, the estimated losses for normal incidence are shown in Table 4.1, and Table 4.2 for concrete and grout, respectively. Table 4.3 , and Table 4.4 show the losses for oblique incidence for concrete and grout respectively.

Table 4.1 Total losses for normal incidence in half-space concrete at $f=915 \mathrm{MHz}$

\begin{tabular}{|c|c|c|c|c|}
\hline \multicolumn{5}{|c|}{ Total Losses for Normal Incidence } \\
\hline Depth, $t$ & $10 \mathrm{~cm}$ & $20 \mathrm{~cm}$ & $30 \mathrm{~cm}$ & $40 \mathrm{~cm}$ \\
\hline Transmission Loss, $A_{t}[\mathrm{~dB}]$ & 0.60 & 0.60 & 0.60 & 0.60 \\
\hline Propagation Loss, $A_{p}[\mathrm{~dB}]$ & 0.26 & 0.51 & 0.77 & 1.02 \\
\hline Total Loss, $A_{\text {TOTAL }}[\mathrm{dB}]$ & 0.86 & 1.11 & 1.37 & 1.62 \\
\hline
\end{tabular}

Table 4.2 Total losses for normal incidence in half-space grout at $f=915 \mathrm{MHz}$

\begin{tabular}{|c|c|c|c|c|}
\hline \multicolumn{5}{|c|}{ Losses in Normal Incidence } \\
\hline Depth, $t$ & $10 \mathrm{~cm}$ & $\mathbf{2 0} \mathbf{c m}$ & $30 \mathrm{~cm}$ & $\mathbf{4 0} \mathrm{cm}$ \\
\hline Transmission Loss, $A_{t}[\mathrm{~dB}]$ & 0.25 & 0.25 & 0.25 & 0.25 \\
\hline Propagation Loss, $A_{p}[\mathrm{~dB}]$ & 0.84 & 1.68 & 2.53 & 3.37 \\
\hline Total Loss, $A_{\text {TOTAL }}($ Half Space $)[\mathrm{dB}]$ & 1.09 & 1.93 & 2.78 & 3.62 \\
\hline
\end{tabular}


Table 4.3 Estimated losses in oblique incidence inside concrete at $f=915 \mathrm{MHz}$

\begin{tabular}{|c|c|c|c|c|}
\hline \multicolumn{5}{|c|}{ Losses in Oblique Incidence } \\
\hline Depth, $t$ & $10 \mathrm{~cm}$ & $20 \mathrm{~cm}$ & $30 \mathrm{~cm}$ & $40 \mathrm{~cm}$ \\
\hline Transmission Loss, $A_{t(||)}[\mathrm{dB}]$ & 2.29 & 2.29 & 2.29 & 2.29 \\
\hline Transmission Loss, $A_{t(\perp)}[\mathrm{dB}]$ & 3.23 & 3.23 & 3.23 & 3.23 \\
\hline Propagation Loss, $A_{p}[\mathrm{~dB}]$ & 0.27 & 0.54 & 0.81 & 1.08 \\
\hline Total Loss, $A_{\text {TOTAL(II) }}[\mathrm{dB}]$ & 2.56 & 2.83 & 3.10 & 3.37 \\
\hline 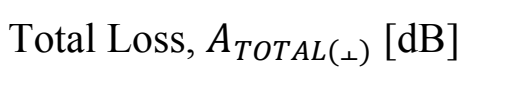 & 3.50 & 3.77 & 4.04 & 4.31 \\
\hline
\end{tabular}

Table 4.4 Estimated losses in oblique incidence inside grout at $f=915 \mathrm{MHz}$

\begin{tabular}{|c|c|c|c|c|}
\hline \multicolumn{5}{|c||}{ Losses in Oblique Incidence } \\
\hline \hline Depth, $t$ & $10 \mathrm{~cm}$ & $20 \mathrm{~cm}$ & $30 \mathrm{~cm}$ & $40 \mathrm{~cm}$ \\
\hline \hline Transmission Loss, $A_{t(\mathrm{II})}[\mathrm{dB}]$ & 2.03 & 2.03 & 2.03 & 2.03 \\
\hline Transmission Loss, $A_{t(\perp)}[\mathrm{dB}]$ & 2.48 & 2.48 & 2.48 & 2.48 \\
\hline Propagation Loss, $A_{p}[\mathrm{~dB}]$ & 0.93 & 1.86 & 2.79 & 3.72 \\
\hline Total Loss, $A_{\operatorname{TOTAL}(\mathrm{I})}[\mathrm{dB}]$ & 2.96 & 3.89 & 4.82 & 5.75 \\
\hline Total Loss, $A_{\operatorname{TOTAL}(\perp)}[\mathrm{dB}]$ & 3.41 & 4.34 & 5.27 & 6.20 \\
\hline
\end{tabular}


After comparing the data provided in Table 4.1, Table 4.2, Table 4.3, and Table 4.4, it can be concluded that concrete presents lesser total loss than concrete in both normal and oblique incidence. Therefore, a communication link is probably more successful inside concrete than grout. These results confirm our expectations based on the experimental results presented in Chapter 3. This study allowed us to predict the behavior of sensors embedded inside concrete and grout in terms of communication range and antenna performance. Therefore, our results enable us to design optimal tags that can be embedded in media, such as, concrete and grout. 


\section{WIRELESS SENSING AND IDENTIFICATION PLATFORM}

The Wireless Identification and Sensing Platform (WISP) is a flexible platform developed by Seattle Intel Research Labs and the University of Washington. The development of WISP was motivated by the RFID industry advances in RFID passive tags. Passive tags are very suitable for long-term monitoring and sensing applications since they can be built without an on-board power supply or battery. Therefore, passive tags are small in size and also have very long lifetime that is equal to the life of the electronic tag. The latest version of WISP is shown in Figure 5.1. A new version, WISP5, is also under development.

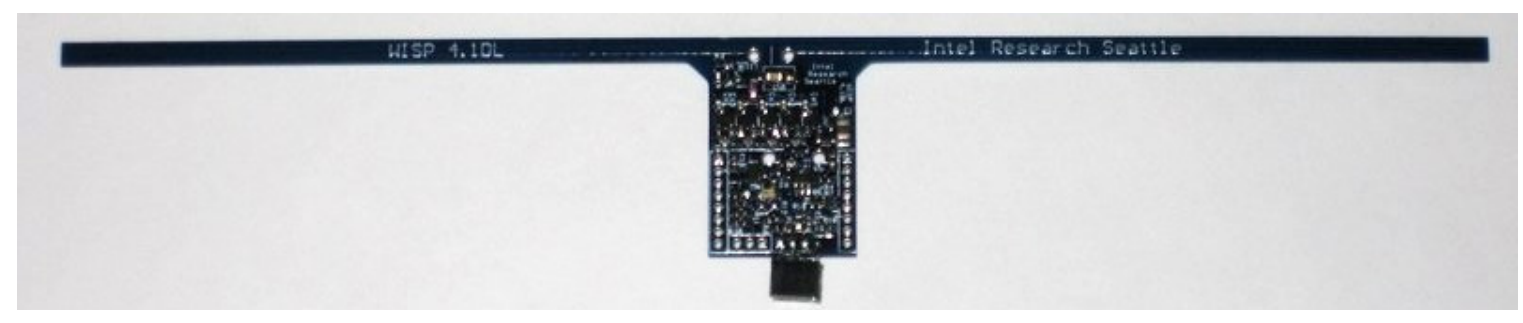

Figure 5.1 Intel WISP version 4.1DL [21]

RFID technology has two well-known standards by EPCGlobal which are Electronic Product Code (EPC) Class 1 Generation 1 and Class 2 Generation 2. These standards usually operate in the Ultra High Frequency (UHF) bands. The same 16 bits EPC code is used by the WISP not only to answer with an identification number but also to transmit data back to the RFID reader. Besides the RFID tag, inside the WISP there is also a 16 bit general purpose microcontroller performing a variety of tasks, such as power management, sampling sensors, computational tasks and transmission of sensed data from the WISP to the RFID reader. However, what makes this platform more 
valuable is its flexibility allowing the use of different sensors for different applications using the same WISP device while it uses no battery. Therefore, WISP works as a traditional passive RFID tag that harvest RF power from the RFID transmitting antenna in order to operate.

The WISP has unique computational capabilities achieved by low power microcontroller and sensors. The WISP printed circuit board (PCB) was implemented with a printed dipole antenna on top of FR4 material. This platform operates by harvesting the RF power sent from the RFID reader for data transmission, and converting it to DC current. After the WISP is powered by the impinging signal from the RFID reader, the microcontroller will collect data from the sensor and send it back together with their unique ID number. Also, the objective behind the WISP design was to create a general platform, instead of a single wireless sensor device. For that matter, a general purpose microcontroller is used, which has additional ports for connecting various sensors. Data transfer between the reader and the RFID tag is accomplished by using Miller 4 encoding and ASK modulation.

This WISP platform is very suitable for measuring temperature, humidity and strain inside a concrete or grout structures. Traditionally, SHM is performed with wired sensors. However, WISP can eliminate not only the use of wires but also the use of batteries altogether. In addition, WISP uses a low power general purpose microcontroller that permits fast code development with debugging support. The following sections discuss in detail the WISP's mode of operation, technology advantages, and maximum operational range. 


\subsection{Operation of WISP}

Similar to any RFID system, WISP communicates with an RFID reader. The RFID reader interrogates the passive tag (WISP) that carries the data retrieving both the identification number and any collected data. This process starts by sending sufficient RF power to WISP. Then, the interrogation sequence works as shown in Figure 5.2. WISP receives the signal, which is connected to the rectifying block on the tag, and converts the $\mathrm{RF}$ power to DC current in order to supply power to its circuitry. Next, the interrogation data is detected by the tag demodulator and the demodulated data is sent to the microcontroller. The microcontroller manages power and collects the data sensed by the sensors. Data collected by the microcontroller is modulated via backscattering modulation and sent back to the RFID reader. The digital modulation used by the WISP is Amplitude Shift-Keying (ASK) modulation. Backscattering radiation or modulation is created by switching the impedance of the front-end of the tag into two known states. In most cases: one is matched to the antenna, usually the state when the power is harvested, and another one where the antenna is strongly mismatched [22]. These states are recognized by the RFID reader and the data is recovered. 


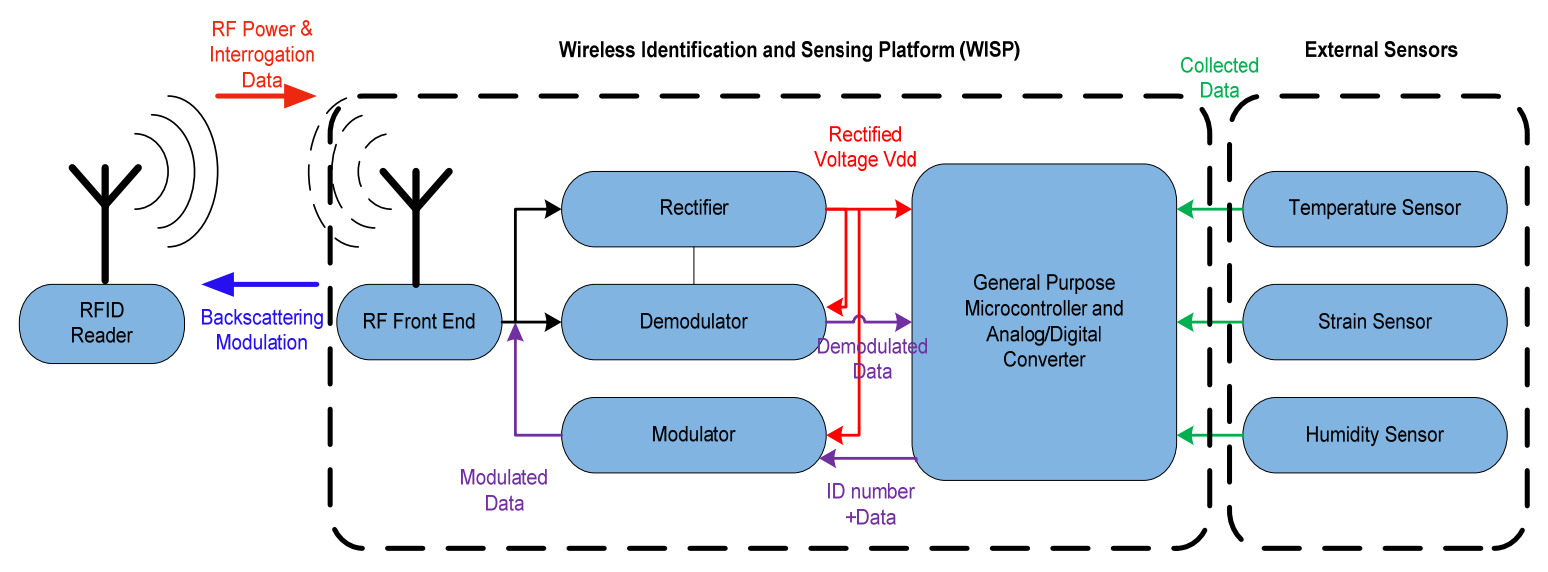

Figure 5.2 WISP block diagram.

The communication link between the reader and WISP is established by either sending a continuous clockwise wave (CWW) to supply power to the WISP or a periodic wave (PW) for interrogation. During the $\mathrm{CW}$ period, the data is backscattered from the WISP into the reader.

\subsection{WISP Hardware}

This section covers the hardware design of WISP, which complies with the EPC Gen 2 Protocol standard and allows successfully collecting and delivering data from the sensors to the RFID reader. Primary, the WISP is built on a four-layer FR4 PCB and traditional Integrated Circuits (ICs) that allow adding and removing sensors depending on the application. WISP is designed so it offers small size, low cost, and customization. Also, it includes a real time clock, an EEPROM memory for saving collected data and, in some cases additional capacitors can be added for applications that need more power. 


\section{$\underline{\text { Receiver Antenna }}$}

The WISP is equipped with an omnidirectional dipole antenna with $2 \mathrm{dBi}$ gain printed on top of an FR4 board. This simple antenna operates at $915 \mathrm{MHz}$. WISP was intended to be used in all directions (i.e., isotropic radiation). Therefore, this antenna meets the requirements of most applications. However, for longer range, a directive antenna will perform better.

\section{$\underline{\text { RF Front End }}$}

Following the dipole antenna, an L-match network is connected between the antenna and WISP circuitry. This L-match network matches the impedances of the tag with the antenna input impedance (72 ohms). This step is critical for maximizing the amount of received RF power by minimizing the mismatch losses between antenna and tag. Similarly, any changes in the WISP antenna requires a redesigning of the L-match network in order to minimize mismatching and achieve maximum efficiency. Inductors used for this L-match network have a high $\mathrm{Q}$ ranging from 30 to 40 (L=1-20nH) for minimizing losses. Also, the antenna is connected to the ASK modulator, which consists of a transistor that changes the antenna's impedance for backscattering radiation.

\section{$\underline{R F \text { Schottky diode rectifier }}$}

The rectifier block was constructed using 5 stages to perform two tasks: (1) conversion from RF power to DC current, and (2) doubling the rectified voltage level. The RF Schottky diodes from Avago HSMS-285x were used for this configuration resulting in an improved input power sensitivity. A more sensitive rectifier block improves the power conversion efficiency and provides better power management. 


\section{Modulator/Demodulator and DC storage}

The output received from the rectifier is detected by the level shifter determining the received symbol and then, simultaneously, the DC current is stored in a capacitor. The demodulator converts the ASK modulated data into a logical binary data. A low level shifter is used to convert the signals from the current linear regulator (On-Semi NCP583) and the demodulators to the microcontroller. When there is a low input power $(<2.8 \mathrm{~V})$, each level shifter consumes less than $500 \mathrm{nA}$. Otherwise, when there is a high input power with voltages up to $6 \mathrm{~V}$, total current consumption is less than $2 \mu \mathrm{A}$. This data is passed to the MSP430 microcontroller that receives the downlink data from the reader and performs tasks according to the firmware already programmed into it. Similarly, for the uplink data, the microcontroller sends the data to the modulator where the backscattering radiation is executed by changing the antenna impedance.

The storage capacitor supplies the necessary power for the microcontroller to operate. A $1.8 \mathrm{~V}$ regulator was added in order to make sure the capacitor has enough power to wake up the microcontroller. Also, a zener diode (On-Semi ESD5Z3.3) is connected in order to prevent the voltage to be larger than $5 \mathrm{~V}$. Additionally, the storage capacitor supplies power to any additional sensors added to the WISP.

\section{Power Management}

Since the amount of available power is very limited, a proper power management mechanism is required in order to guarantee reliable operation of WISP tags. Also, the firmware is active for only a few milliseconds before the microcontroller resets due to low voltage. Consequently, WISP operates in active and sleep mode cycles that are 
managed by the microcontroller. In sleep mode, the WISP is turned off resulting in a reduction of the current consumption to a few microamperes and also allowing the capacitor to recharge. Once sufficient voltage is stored (around $1.8 \mathrm{~V}$ ), the microcontroller is "woken up" by $1 \mu A$ quiescent current linear regulator. For most tasks, the necessary voltage for operation is $2 \mathrm{~V}$, which is detected by a $300 \mathrm{nA}$ quiescent current linear supervisor. This supervisor wakes up the microcontroller for operation instead of the microcontroller periodically polling for sufficient voltage. Once the microcontroller has sufficient power, it starts polling the sensors, collecting and storing data, and communicating with the reader.

\section{$\underline{\text { Communication }}$}

WISP detects the modulated data from the envelope of the RF carrier signal. The demodulator compares the output rectified voltage to a peak value used as reference. An additional $1 \mu \mathrm{F}$ capacitor is connected to the comparator for both reference voltage and supplying power to the comparator.

\subsection{WISP Firmware}

A critical component in the WISP hardware design is the addition of computational capabilities by using a low power general purpose microcontroller MSP430 by Texas Instruments. Therefore, a firmware, which controls several tasks was developed for the WISP. The WISP firmware can be downloaded from the Github repository [23]. This repository offers three codes (1) a firmware for programming the

WISP 4.1 (C code), (2) a C\# code for controlling the Impinj Speedway RFID reader, and (3) a Verilog code for testing the RFID tag. Basically, the WISP code is compiled and 
downloaded into the WISP via the IAR Embedded Workbench Kickstart Edition MSP430 v5.4 or lower version. The interface between the personal computer and the WISP is made by a USB Key debugger as shown in Figure 5.3.

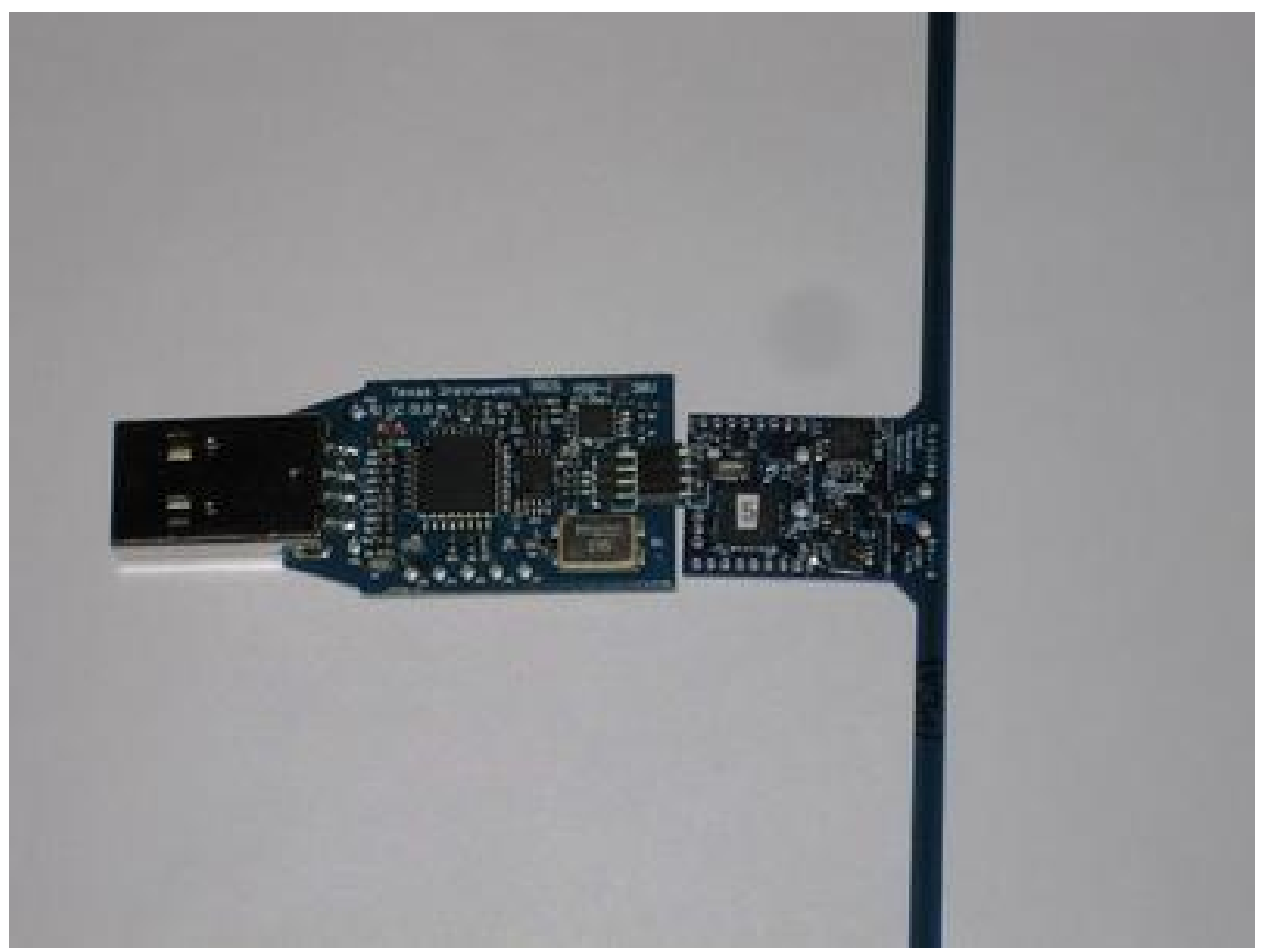

Figure 5.3 USB Key Debugger and WISP for programming. (Photo from [21])

On the other hand, the RFID reader needs an interface that can recover the data collected from the WISP and correctly display it for users. Therefore, in that sense, in order to run the RFID reader an LLRP Visual Studio C\# App created by Intel was created and it was used for this research as shown in Figure 5.4. 


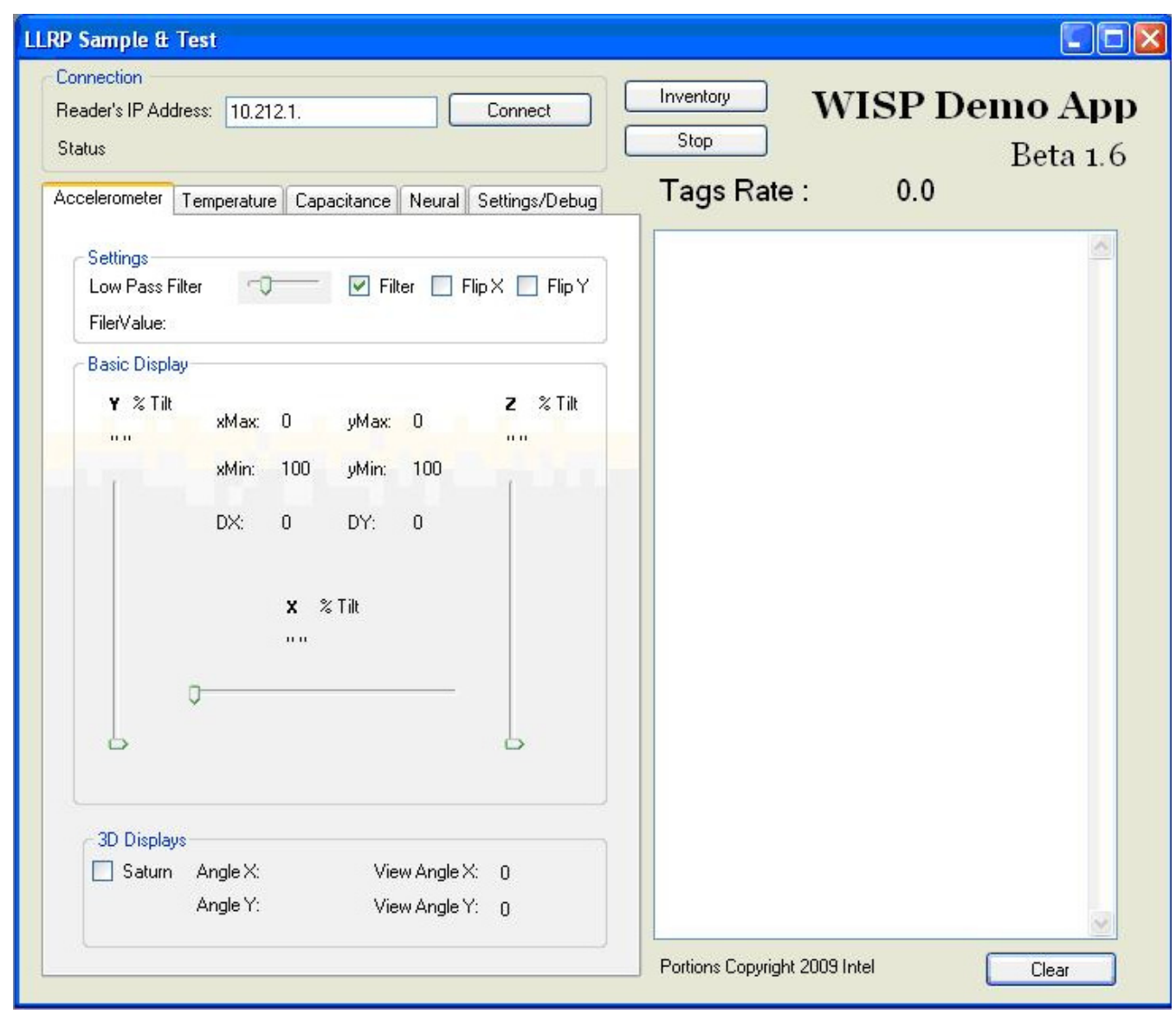

Figure 5.4 WISP demo application for running the RFID reader.

Figure 5.5 provides a firmware block diagram, which illustrates how the three main tasks performed by the WISP are linked to the WISP's hardware. These three tasks are: (1) power management that keeps the system operating when voltage levels are low, (2) implement the EPC Class 1 Gen 2 protocol for transmitting and receiving data, and (3) run applications on the WISP. Each task will be briefly described next. 


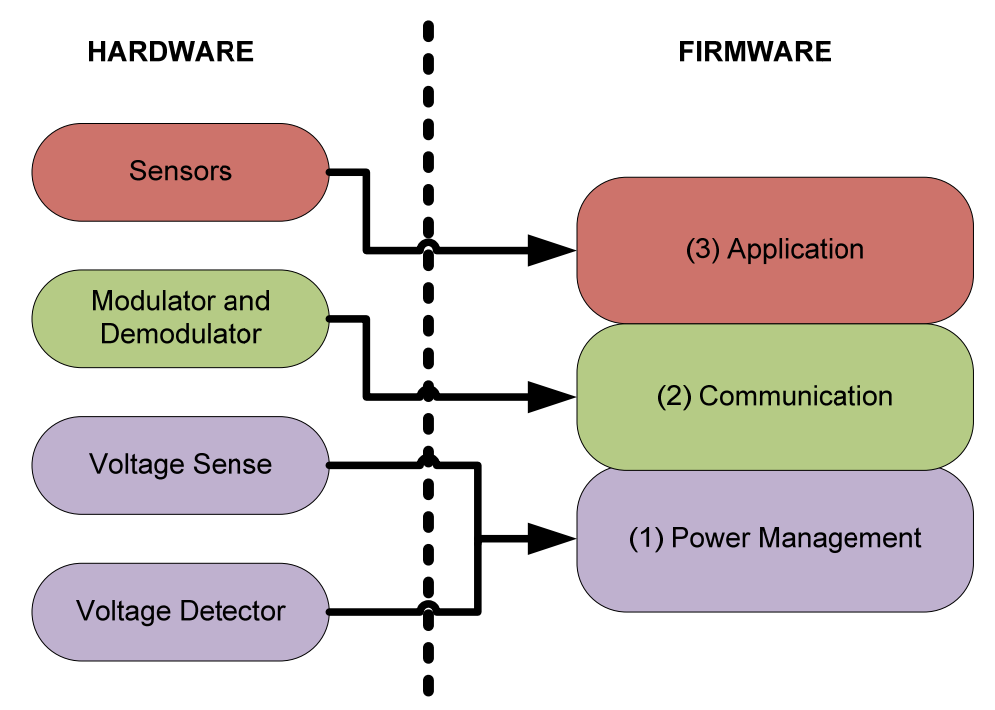

Figure 5.5 Firmware block diagram.

The power management part of the firmware controls the active and sleep cycles. Based on the detected and available sensed voltage, the software puts the microcontroller on either sleep or active mode. The sleep cycles are critical for recharging the storage capacitor supplying the power for the microcontroller and the sensors. The communication level relies on the power management algorithm. During the active mode operation, the firmware performs both downlink and uplink communications following Gen2 protocol. Finally, the applications level is dedicated to perform user's customized functions, such as sensor measurements, and encoding data in EPC packets.

\subsection{Power Budget}

Like any communication technology, the WISP operation faces various limitations. First, the RFID readers must comply with regulations established by the regional regulators, such as, the Federal Communication Commission (FCC) in North America. FCC regulates that the allowable RF power for Industrial, Scientific, and 
Medical (ISM) band should not overcome 4W Equivalent Isotropic Radiation Power (EIRP). EIRP refers to an ideal isotropic antenna radiating equally in all directions. Therefore, the maximum permitted power transmitted from the RF reader would be determined by (5.1):

$$
\operatorname{EIRP}=P_{T}-L_{C}+G_{a}
$$

where $P_{T}$ is the reader output power, $L_{C}$ is the cable losses and $G_{a}$ is the antenna gain. In most cases $L_{C}$ is really small and therefore neglected. In that case, choosing an antenna gain will fix the output power for RFID operation, according to the FCC standard.

Since passive tags harvest the power from the interrogating signal transmitted by the RFID reader, the received power, $P_{R}$, at the tag is constrained by the RFID reader's power and the path losses. Additionally, if the tags are embedded inside a material different than air, transmission and propagation losses, $A_{t}$, and, $A_{p}$, should be accounted as well. The Friis expression explains this relationship as:

$$
P_{R}=P_{T}+G_{T}+G_{R}-20 \log \left(\frac{4 \pi d}{\lambda}\right)-L_{P}-A_{\text {Total }}
$$

where $G_{T}$ and $G_{R}$ are the gains of the transmitting and receiving antennas, respectively. The expression $20 \log \left(\frac{4 \pi d}{\lambda}\right)$ represents the path loss, which corresponds to losses based on the separation or distance, $d$, between the reader and the tag. $L_{P}$ accounts for the polarization losses between the antennas, and $A_{\text {Total }}$ represents the total losses suffered by a waveform propagating through materials different than air. In other words, the amount of power available to the tag for harvesting depends on the distance between the two antennas and the amount of losses that the material where the tag is embedded in 
introduces. Therefore, based on what was explained before, Table 5.1 shows the parameters used to operate WISP inside concrete and grout.

Table 5.1 Power budget for WISP operation

\begin{tabular}{|c|c|c|}
\hline Parameters & RFID Reader & WISP \\
\hline \hline Wavelength, $\lambda[\mathrm{m}]$ & 0.33 & - \\
\hline Transmission Power, $\mathrm{P}_{\mathrm{t}}[\mathrm{dBm}]$ & 30 & - \\
\hline Transmitter Antenna Gain, $\mathrm{G}_{\mathrm{t}}[\mathrm{dBi}]$ & 8 & 2 \\
\hline Receiver Antenna Gain, $\mathrm{G}_{\mathrm{R}}[\mathrm{dBi}]$ & - & \\
\hline Polarization Loss, $L_{p}[\mathrm{~dB}]$ & \multicolumn{2}{|}{} \\
\hline
\end{tabular}




\section{EXPERIMENTAL ANALYSIS}

In this chapter, two experimental setups were conducted in order to assess the performance of WISP inside concrete and grout. First, the maximum range of operation of the WISP inside concrete and grout were experimentally determined. It became vital to understand the maximum ranges at which WISP can still collect data and communicate with the RFID reader. Second, the power transfer efficiency between the RFID antenna and the WISP was experimentally determined in order to provide insight regarding the optimum distance, $d$, between the antennas and optimum depths, $t$, where WISP tags should be placed at in concrete and grout. The concrete and grout samples used for our experiments had the following dimensions: (1) $\mathrm{cm}$ thickness of $10 \mathrm{~cm}$, (2) height of 10 $\mathrm{cm},(3)$ and length of $40 \mathrm{~cm}$.

Each experimental setup used the following equipment: (1) Impinj Speedway Revolution R220 UHF Gen 2 RFID reader (see Figure 6.1), (2) Laird Cushcraft S9028PLC12NF 8 dBi RFID antenna (see Figure 6.2), and (3) Vector Network Analyzer HP8722 (see Figure 6.3) 


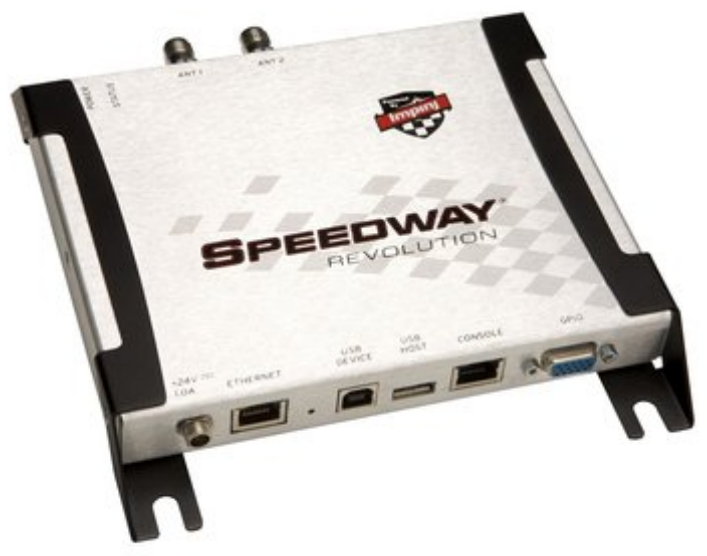

Figure 6.1 Impinj Speedway R220 RFID reader.

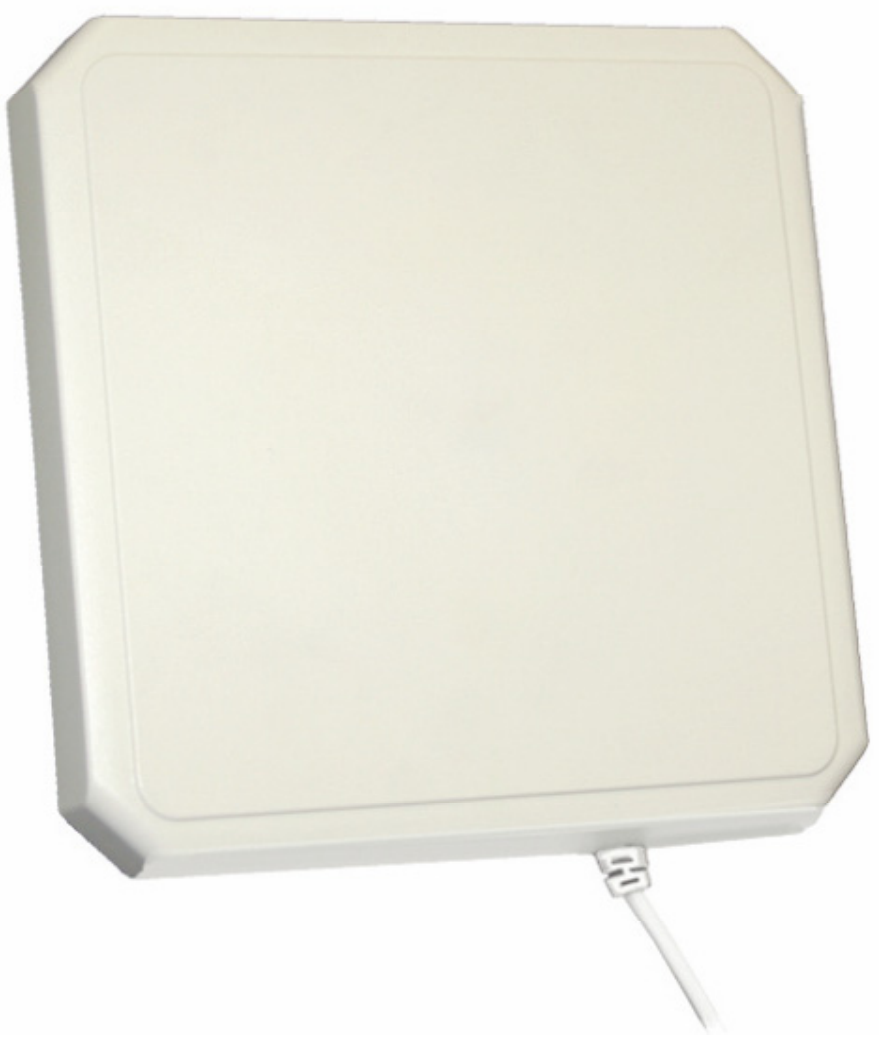

Figure 6.2 Laird Cushcraft 902-928 MHz 8 dBi Circularly Polarized Antenna. 


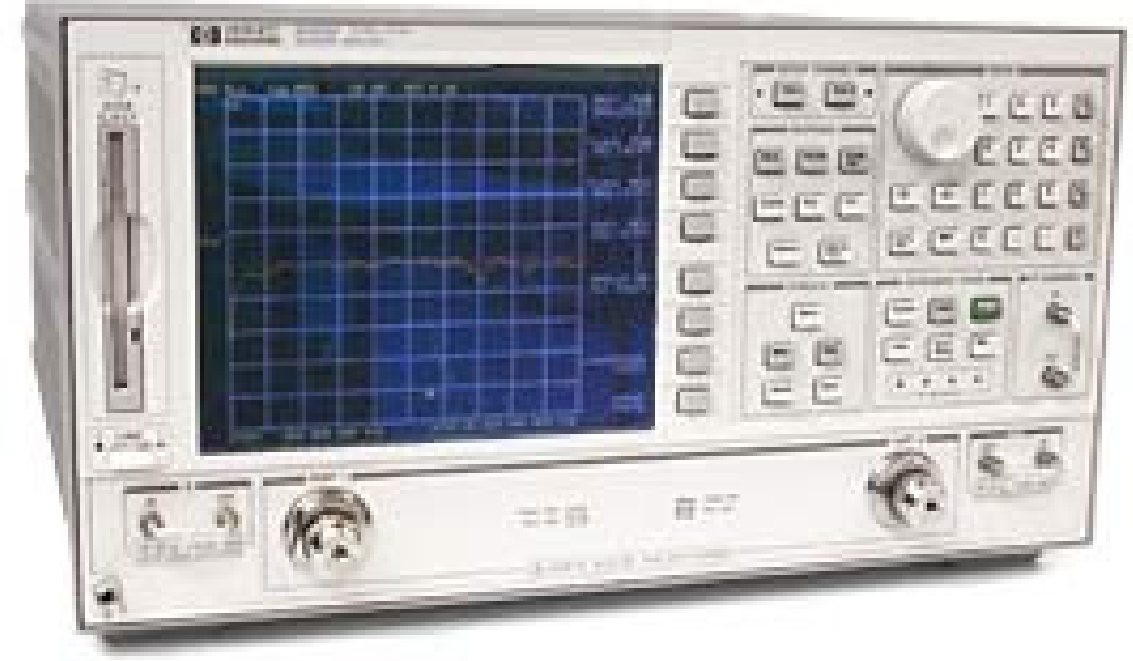

Figure 6.3 HP 8722 Vector Network Analyzer

\subsection{WISP maximum range of operation inside concrete and grout}

In this first section, the maximum operating range for WISP was determined for concrete and grout. From a design point of view, it is considered beneficial to understand the maximum range where the WISP can still perform measurements and communicate with the reader for delivering the data. Several blocks of concrete and grout were made for this experiment. The designed block diagram and the real experimental setup are shown in Figure 6.4, and Figure 6.5, in that order. In this setup, a fixed thickness, $t_{f}$, concrete or grout sample was positioned behind the WISP tag. A sample with variable depth, $t$, was positioned between the WISP and the RFID antenna. The sample depth was varied from $10 \mathrm{~cm}$ to $40 \mathrm{~cm}$ with steps of $10 \mathrm{~cm}$. The RFID antenna/reader was connected to a personal computer displaying data from the WISP. Finally, the RFID reader was fixed at the same position, and only WISP was moved. 
After setting the experiment, two cases were studied: (a) determine the maximum radial communication range, $r$, between the WISP and the RFID antenna; and (b) determine the maximum axial range, $a$, by positioning the RFID antenna flash to the surface of concrete or grout and by moving the WISP axially.

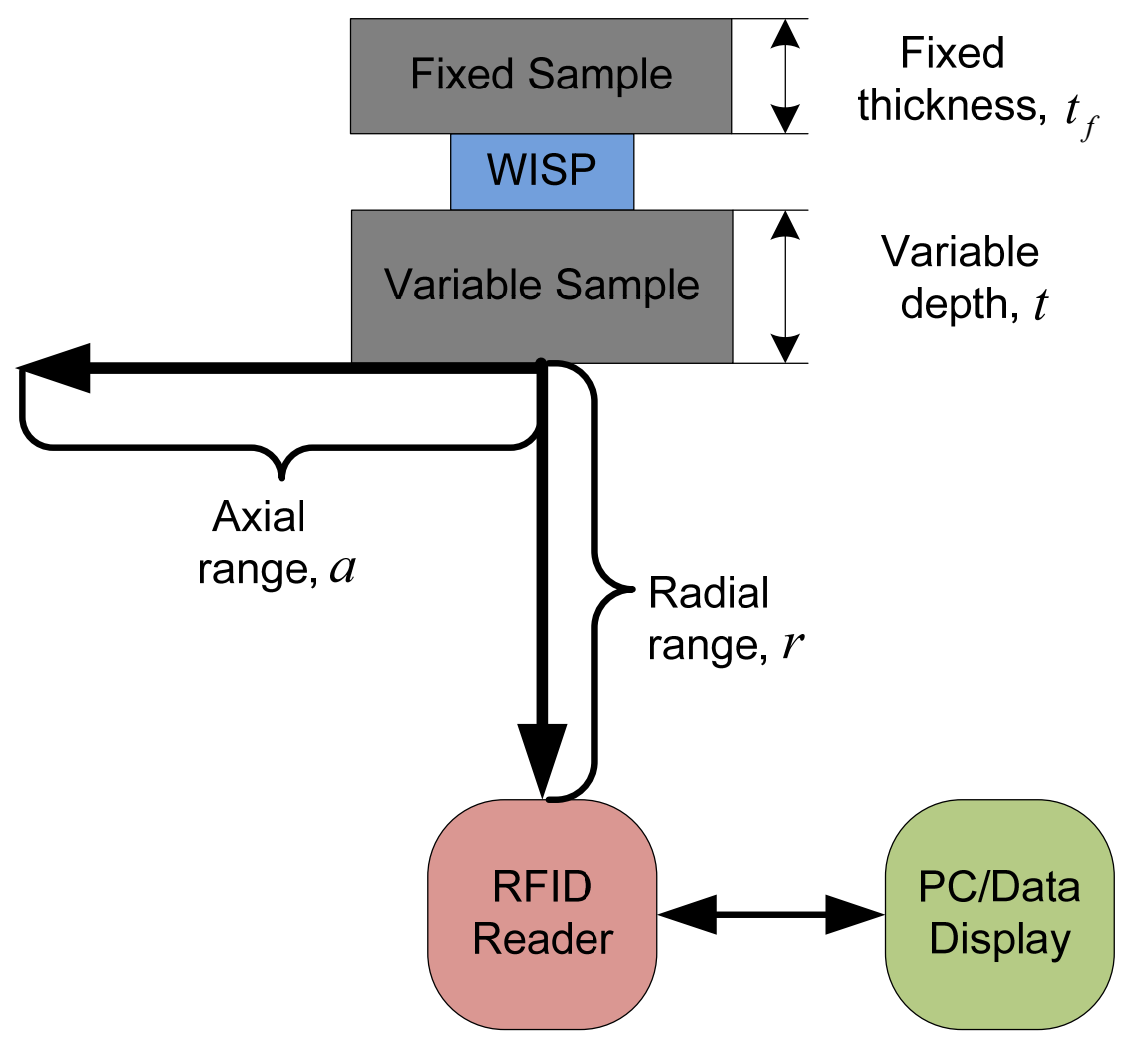

Figure 6.4 Experimental setup for measuring maximum axial range, $a$, and radial range, $r$, of WISP inside lossy materials. 


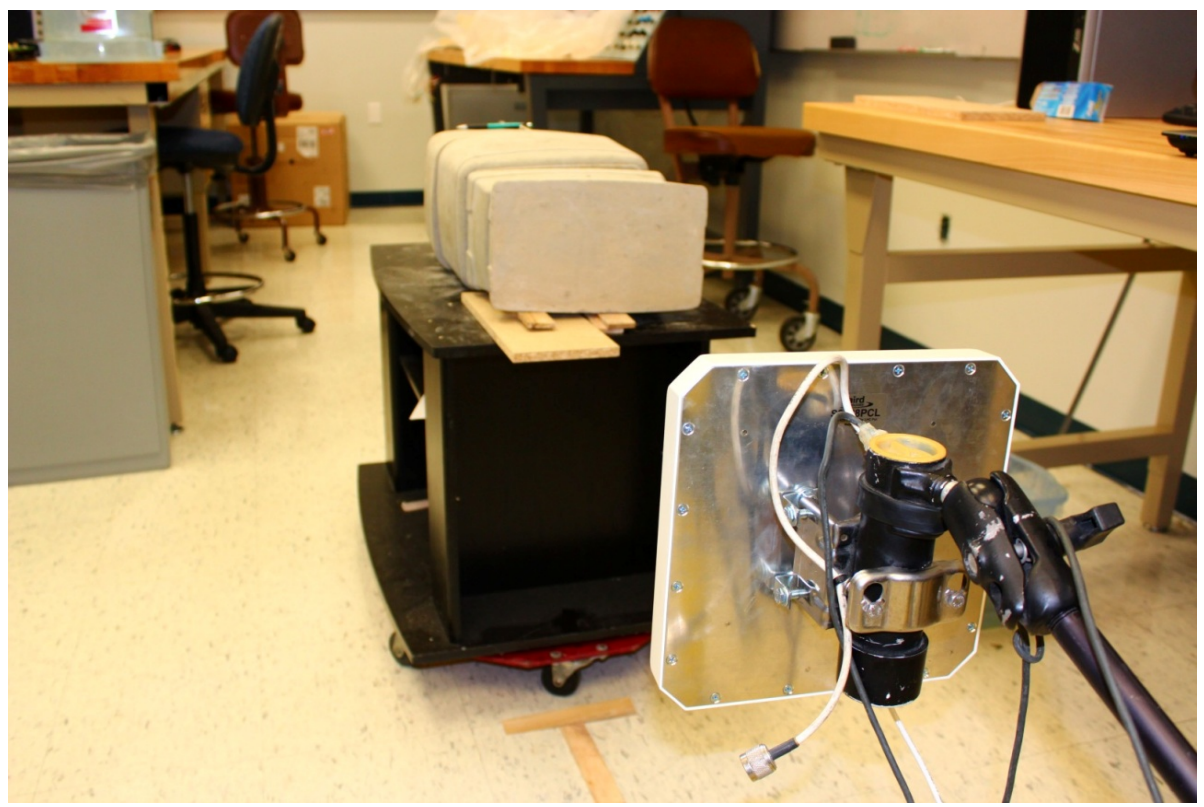

Figure 6.5 Actual experimental setup.

For both cases WISP was programmed to measure temperature and the data was displayed in a personal computer (PC). The RFID antenna was moved until the tag rate of the WISP was near zero, which means that no data was being sent back from the WISP. The experimental results regarding the radial and axial ranges for both concrete and grout are shown in Table 6.1, and Table 6.2, respectively.

Table 6.1 Maximum radial and axial ranges of WISP inside concrete for different depths

\begin{tabular}{|c|c|c|c|c||}
\hline \multicolumn{1}{|c|}{} & \multicolumn{4}{c||}{ Depth, } \\
\cline { 2 - 5 } & $10 \mathrm{~cm}$ & $20 \mathrm{~cm}$ & $30 \mathrm{~cm}$ & $40 \mathrm{~cm}$ \\
\hline \hline Radial range, $r$ & $1.29 \mathrm{~m}$ & $0.63 \mathrm{~m}$ & $0.60 \mathrm{~m}$ & $1.24 \mathrm{~m}$ \\
\hline Axial range, $a$ & $0.38 \mathrm{~m}$ & $0.46 \mathrm{~m}$ & $0.50 \mathrm{~m}$ & $0.53 \mathrm{~m}$ \\
\hline
\end{tabular}


Table 6.2 Maximum radial and axial ranges of WISP inside grout for different depths

\begin{tabular}{|l|c|c|c|c||}
\hline \multirow{2}{*}{} & \multicolumn{4}{|c|}{ Depth, } \\
\cline { 2 - 5 } & $10 \mathrm{~cm}$ & $20 \mathrm{~cm}$ & $30 \mathrm{~cm}$ & $40 \mathrm{~cm}$ \\
\hline \hline Radial range, $r$ & $1.29 \mathrm{~m}$ & $0.69 \mathrm{~m}$ & $1.18 \mathrm{~m}$ & $0.83 \mathrm{~m}$ \\
\hline Axial range, $a$ & $0.75 \mathrm{~m}$ & $0.51 \mathrm{~m}$ & $0.36 \mathrm{~m}$ & $0.21 \mathrm{~m}$ \\
\hline
\end{tabular}

These results established the feasibility of performing measurements using WISP tags at average ranges between $0.6 \mathrm{~m}$ to $1.29 \mathrm{~m}$ inside both concrete and grout. The existence of standing waves caused the radial and/or the axial ranges to oscillate between larger and smaller values for increasing depths. In summary, our results illustrate that battery-less WISP tags can be used to monitor quantities, such as, temperature, humidity, or strain inside concrete or grout, and achieve this for the ranges shown in Table 6.1 and Table 6.2.

While conducting this experimental setup, temperature was measured using the internal temperature sensor implemented with WISP. It can be observed in Figure 6.6 that more temperature samples were being received when the WISP was closer to the RFID reader antenna. This means that WISP had more available power and exchanged more information with the RFID reader. 


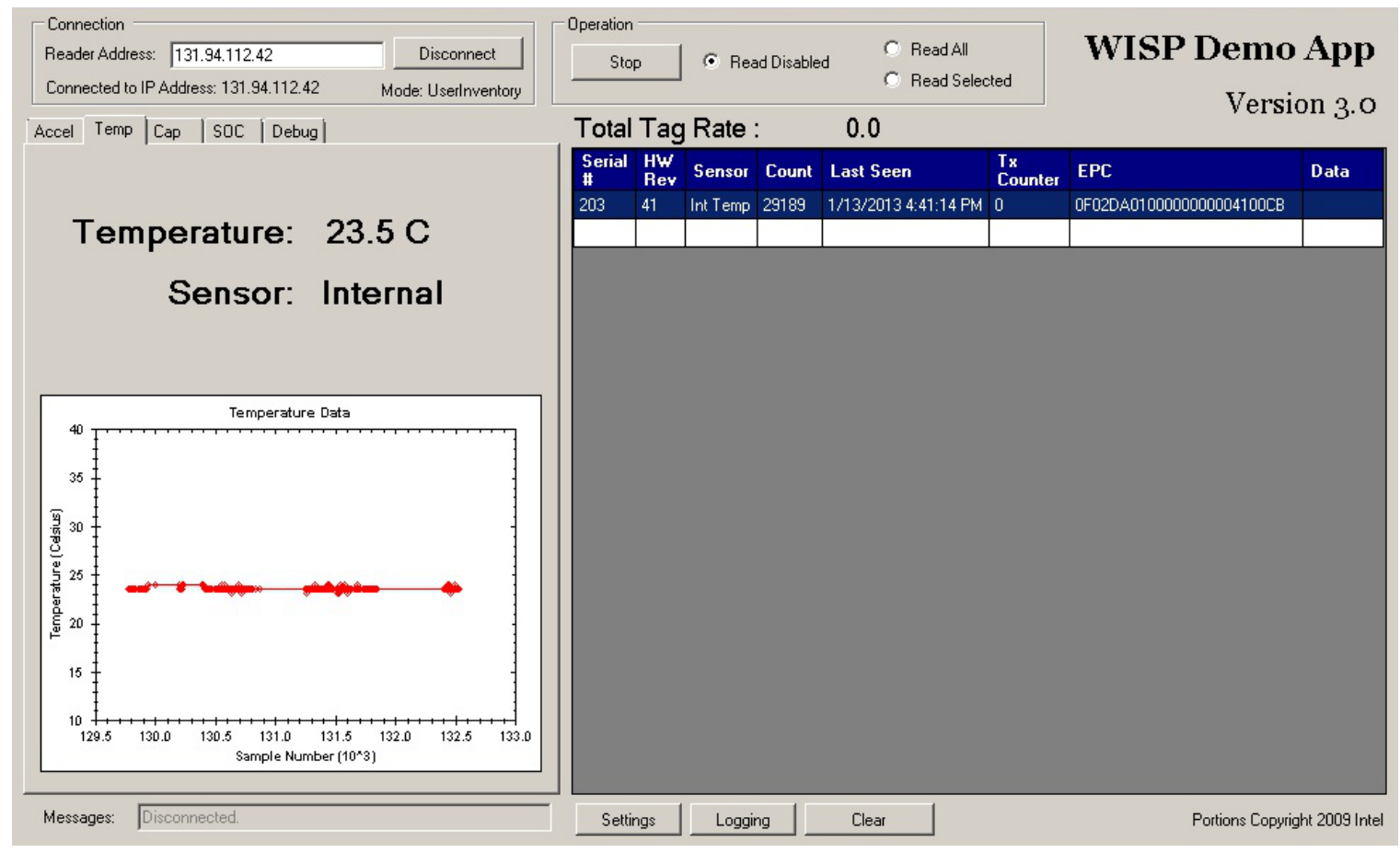

Figure 6.6 Temperature measurement with the WISP inside concrete.

\subsection{WISP Power Transfer in an RFID System}

In this section, the RF power transfer between the WISP and the RFID reader antenna (i.e., transmission coefficient $S_{12}$ ) was studied while WISP was embedded inside (1) concrete with different depths, $t$, and (2) grout with different depths, $t$. In order to measure the S-parameters, the RFID reader antenna was connected to one port of a vector network analyzer (VNA) while the WISP antenna was connected to the second port. In order to improve the accuracy of these measurements, the WISP was programmed to be in sleep mode in order to reduce the power consumption of the WISP. In order to conduct the measurements, an SMA connector was soldered at the dipole feed point of the WISP.

Next, the WISP was placed in front of a concrete or grout sample with fixed thickness $t_{f}$ of $10 \mathrm{~cm}$. In front of WISP, another sample of concrete or grout with 
variable depth, $t$, was placed. Depth, $t$, takes values of $10 \mathrm{~cm}, 20 \mathrm{~cm}, 30 \mathrm{~cm}$, and $40 \mathrm{~cm}$. For depth, $t$, (i.e., $10 \mathrm{~cm}$ ), the distance between the RFID antenna and WISP, $d$, varied from $0 \mathrm{~cm}$ to $45 \mathrm{~cm}$. The transmission coefficient $\mathrm{S} 12$ between the antennas was measured for different distances, $d$, at operational frequency $f=915 \mathrm{MHz}$, as shown in Figure 6.7. Fig. 3 and 4 show the real experiment perfomed on both concrete and grout, respectively.

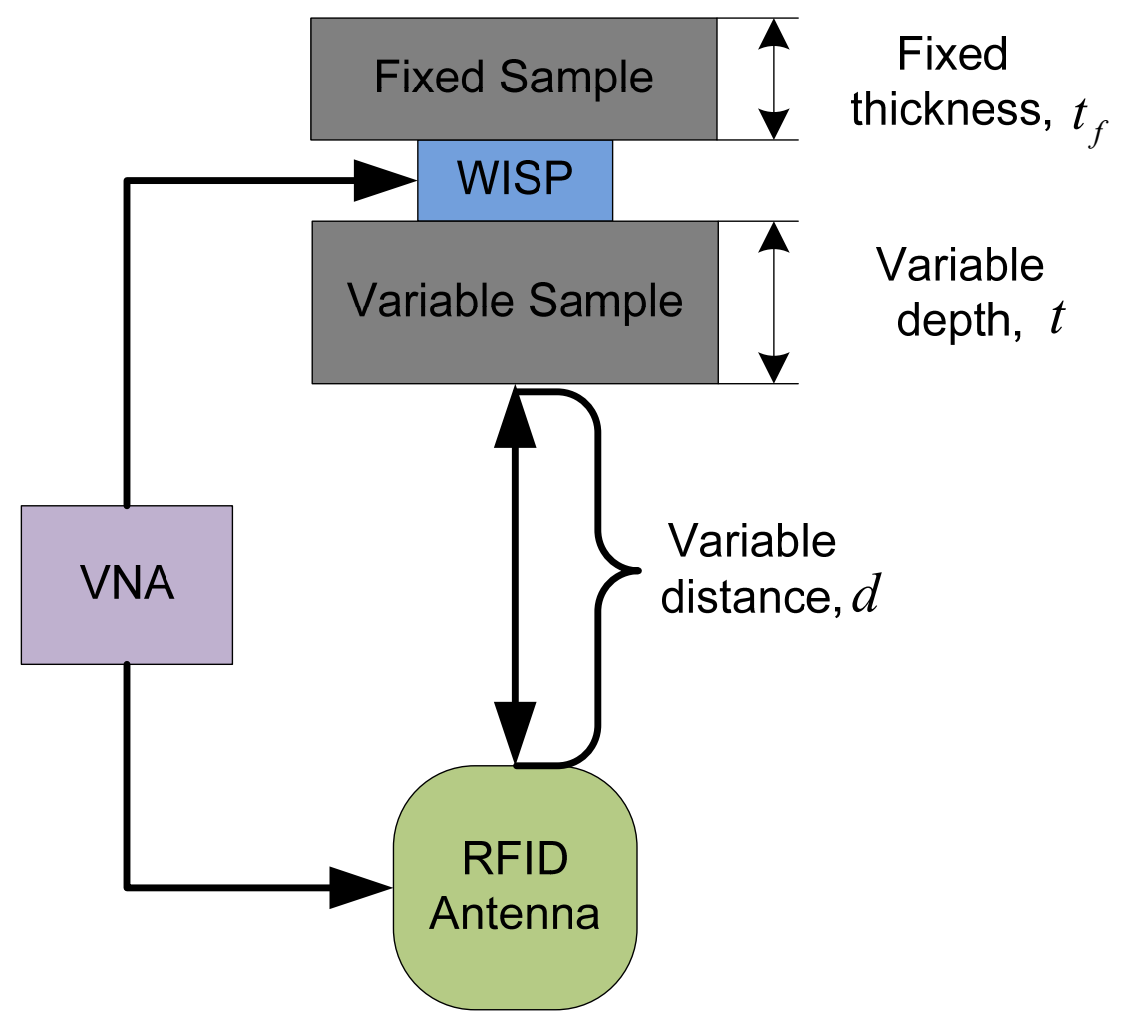

Figure 6.7 Experimental setup for measuring the transmission coefficient $S_{12}$ between the RFID antenna and the WISP tag for variable distance, $d$. 


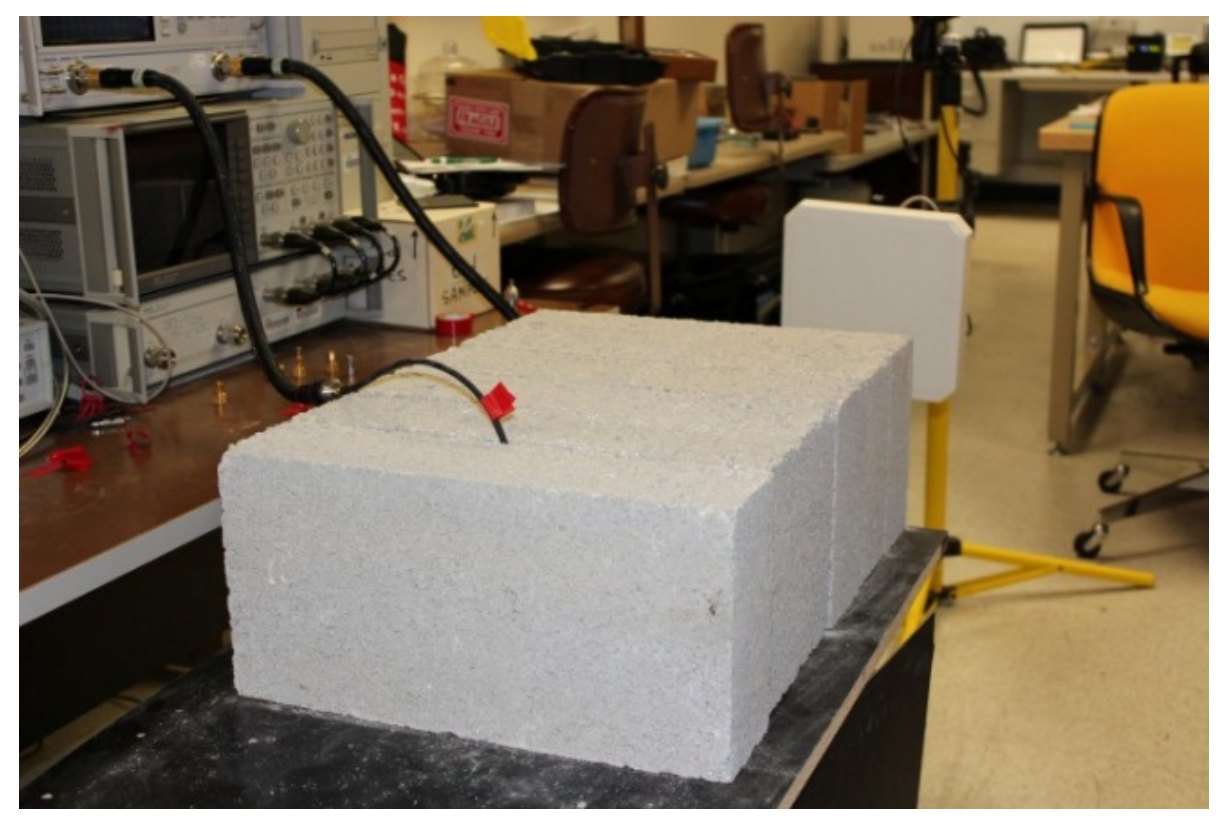

Figure 6.8 Power transfer experimental setup for the WISP inside concrete at different depths, $t$.

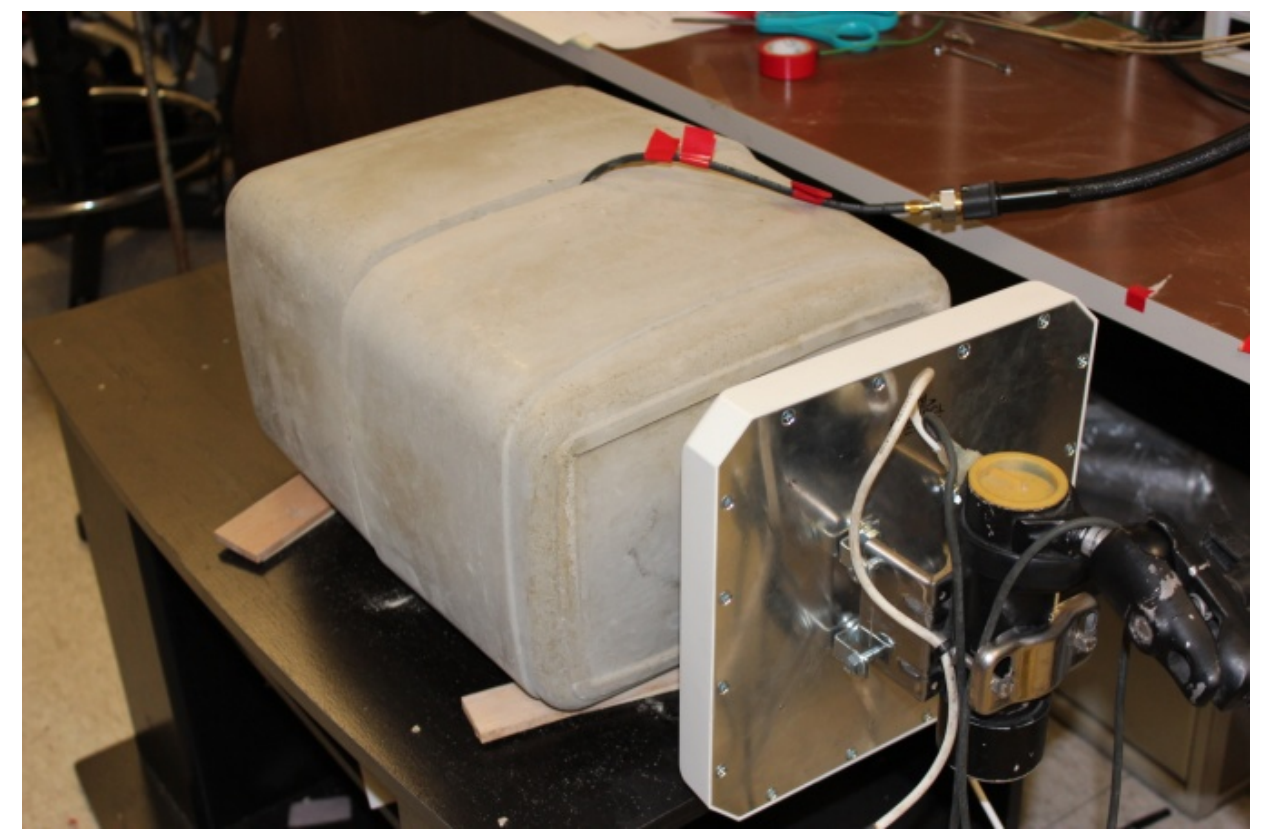

Figure 6.9 Power transfer experimental setup for the WISP inside grout at different depths, $t$. 
The measurements for concrete are summarized in Figure 6.10. It displays the behavior of the transmission coefficient, $S_{12}$, between the antennas versus distance, $d$, for different placement depths, $t$, of the WISP inside concrete. A higher $S_{12}$ is equivalent to larger efficiency of power transmission between the RFID antenna and the WISP antenna. It can be seen that the best coupling between the antennas can be achieved for $d$ around 5 to $10 \mathrm{~cm}$. It should be also pointed out, that in the case where the RFID antenna was positioned against the material surface; the antenna coupling was the least. Also, when the distance, $d$, increases, the coupling between the antennas oscillates thereby behaving similarly to a standing wave formed by transmitted and reflected waves, between the RFID reader antenna and the WISP tags. These standing waves are caused by the presence of two materials (i.e., air and concrete)

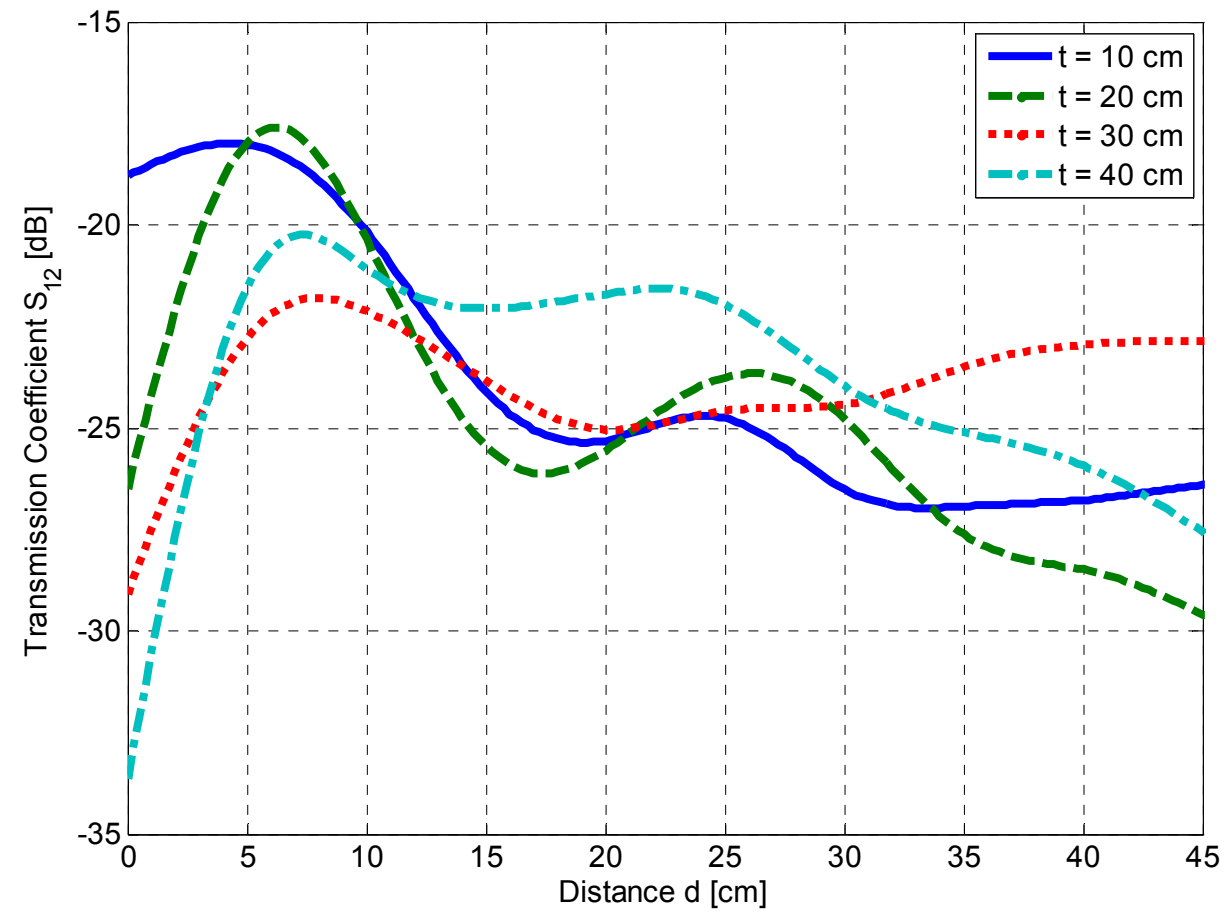

Figure 6.10 Coupling between the RFID antenna and the WISP in concrete at different depths, $t$, versus distance, $d$, at $f=915 \mathrm{MHz}$. 
Subsequently, the same measurements were conducted for grout and they are presented in Figure 6.11. It can be seen that the more adequate distance, $d$, of approximately $5 \mathrm{~cm}$ to $10 \mathrm{~cm}$ provides the best coupling between the antennas. Both Figure 6.10, and Figure 6.11 also confirm the data provided in Table 6.1, and Table 6.2 that showed that concrete experiences smaller losses than grout at $915 \mathrm{MHz}$. Therefore, WISP tags embedded in concrete receive more power than WISP tags embedded in grout, which in turn explains the determined axial and radial ranges presented in Table 6.1, and Table 6.2. Also, obtained results confirm that concrete is less affected by losses than grout which validate the losses calculated in Table 4.1, Table 4.2, Table 4.3, and Table 4.4.

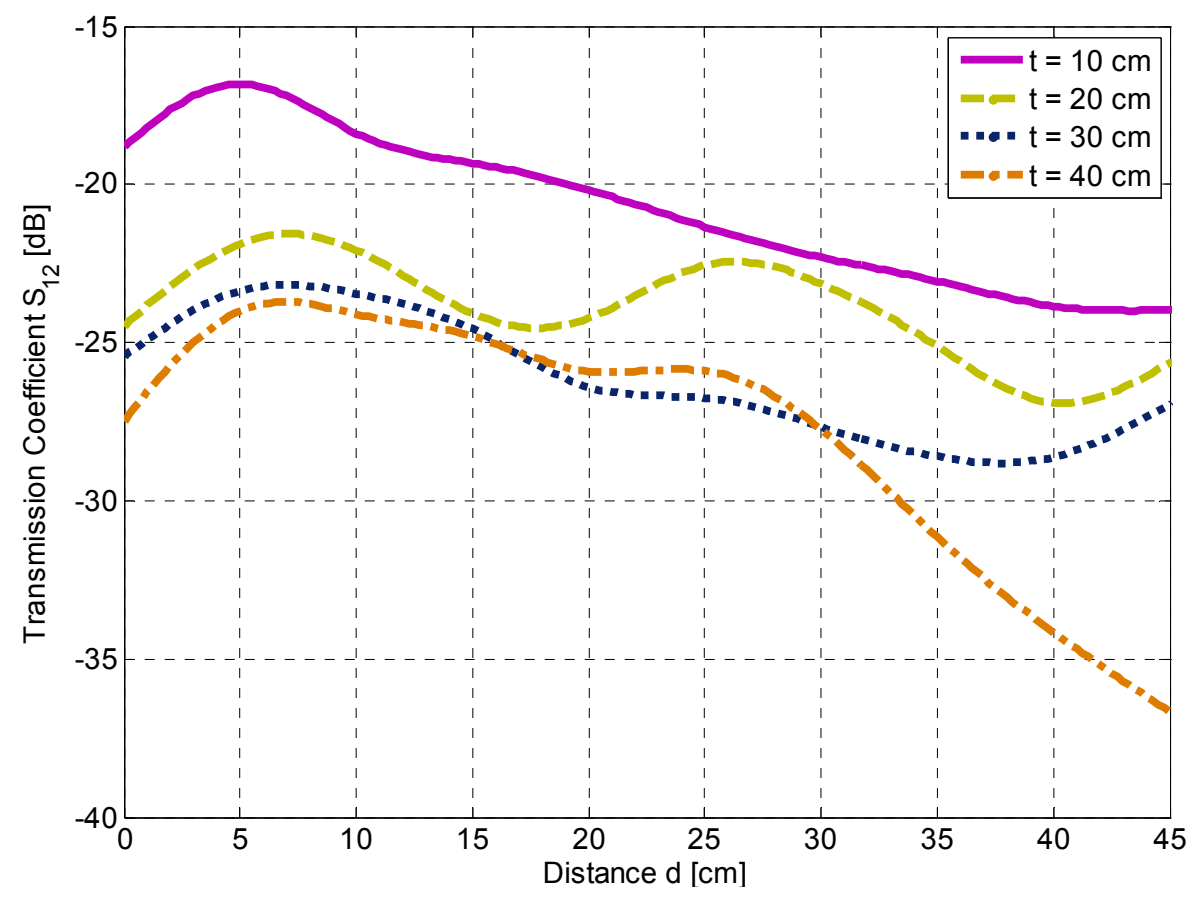

Figure 6.11 Coupling between the RFID antenna and the WISP in grout at different depths, $t$, versus distance, $d$, at $f=915 \mathrm{MHz}$. 


\subsection{Discussion of Results}

The communication range between a WISP tag and a commercial RFID reader antenna was measured for both concrete, and grout. Our experimental data confirms the use of WISP tags for monitoring temperature, humidity and strain inside lossy media, such as, concrete and grout. It was determined that WISP will continue to operate in a range of $0.6 \mathrm{~m}$ to $1.29 \mathrm{~m}$ for different depths, $t$, of both materials concrete and grout. Further measurements were conducted in order to determine the power transfer between a WISP and a commercial RFID reader antenna; these data confirmed the oscillatory behavior of the coupling due to the existence of standing waves. This research is a first step in confirming the effective use of battery-less WISP tags for monitoring nuclear plants for decommissioning tasks. Also, WISP tags can be used in Structural Health Monitoring (SHM) of buildings and concrete structures. 


\section{CONCLUSIONS AND FUTURE WORK}

As part of the American Recovery and Reinvesting Act, DOE is involved in evaluating new technologies and methods for decommissioning nuclear plants. Current decommissioning methods are costly and time consuming. Therefore, the entombment of nuclear plants is a better solution compared to packaging and transporting nuclear waste to other locations. Entombment of nuclear plants requires monitoring of these isolated structures for security and environmental reasons. Migrating from wired to wireless sensors is not only less expensive but also a feasible solution. The application of wireless communications and powering of wireless sensors embedded into lossy media, such as concrete and grout have been examined by this work. Also, these materials were studied and modeled. Finally, WISP was tested in experimental setups in order to identify its operational axial and radial ranges as well as antenna efficiency.

This research experimentally measured the electrical properties of novel grouts intended for use in decommissioning of nuclear plants. Also, concrete was examined to validate our measurement process. Differences between the dielectric permittivity of concrete and grout caused different losses to RF signals that travel through these materials. Both materials, concrete and grout, were modeled as a Debye dispersive material and losses were calculated from $50 \mathrm{MHz}$ to $1 \mathrm{GHz}$. Also, the transmission and propagation losses of RF signals were calculated versus frequency for both materials. Even though this study focused on the operational frequency of $915 \mathrm{MHz}$, experimental results from this work can be used for applications using frequencies ranging from 50 $\mathrm{MHz}$ to $1 \mathrm{GHz}$. Our results showed that RF waves propagating through grout experience 
smaller losses at lower frequencies. Also, this work demonstrated that wireless sensors embedded in concrete suffer less losses than those embedded in grout. Therefore, the design of new decommissioning materials that differ from concrete can possibly use this information for creating decommissioning materials that have lower losses.

Another important aspect about this research is that it presents the first batteryless SHM system for decommissioning plants. RFID technology-based WISP created by Intel Research Labs and University of Washington offers a flexible battery-less platform that can measure quantities such as temperature, humidity, and strain. In fact, to our knowledge, no previous study has covered WISP performance inside lossy media, such as concrete and grout. Specifically, this work experimentally tested WISP tags inside concrete and grout and determined the maximum radial and axial communication ranges. For different depths, $t$, samples of both materials were positioned between WISP and the RFID reader antenna proving that WISP continues to operates in distances from $0.6 \mathrm{~m}$ to $1.29 \mathrm{~m}$. These results prove that WISP can be used for SHM applications. Additionally, in order to potentially improve these operational ranges, the transmission coefficients, $S_{12}$, between the antennas were experimentally. The transmission coefficient, $S_{12}$, describes the efficiency of power transfer between the antennas. Our experimental results show the following:

1. Positioning the RFID antenna directly against the material presents the lower coupling or power transfer efficiency between the RFID antenna and the WISP dipole. 
2. An adequate distance ranging from 5 to $10 \mathrm{~cm}$ provides higher coupling between the antennas. Therefore, this should be the distance between the RFID antenna and the WISP tags for optimal communication links and recharging.

3. The transmission coefficient, $S_{12}$, versus distance, $d$, show an oscillating behavior as result of the superposition of transmitted and reflected waves inside the concrete and grout samples. Therefore, the best positioning of the sensors depend on the wavelength of the operational frequency and the placement depth, $t$.

This work studied the materials properties of concrete and grout, modeled the EM losses inside such materials, and evaluated the performance of battery-less WISP tags inside them. Future work can include the use of a directional patch antenna on WISP tags. The current dipole antenna that is used in WISP has an omnidirectional radiation pattern, which wastes power emitting in all directions. A directional antenna will allow the collection of more power from the direction of the RFID antenna thereby improving the transmission coefficient $S_{12}$ between the antennas, increasing the communication range, and providing more power to the WISP tag. Therefore, WISP tags will have more power to spend on their sensors. Changes in the antenna design of WISP will need to be accompanied by changes in the impedance matching network. Another aspect that will need to be addressed in the future will be the proper packaging of the WISP tag for protecting the circuitry when it is embedded in concrete or grout. One possibility will be to use a two-part urethane potting that cures at room temperature and exhibits chemical resistance thereby not allowing the material to damage the tag's electronics. 
Finally, since WISP has been traditionally used to measure temperature and acceleration based on its standards sensors, new sensors can be incorporated in the future that can measure other quantities, such as strain and humidity inside concrete and grout. For this matter, low power (around 1.8 volts) humidity and strain sensors will need to be used. In order to incorporate such sensors in WISP tags is necessary to implement a signal conditioning board that includes a low signal amplifier and a capacitive bridge (for humidity sensors), or a Wheatstone bridge (for strain sensors). After designing the new board and connecting it to the WISP, a firmware and GUI will have to be developed in order to display the results in a personal computer. This will provide a user-friendly interface for users to monitor the collected sensed data by battery-less WISP tags in concrete and grout for SHM applications. 


\section{BIBLIOGRAPHY}

[1] J. Samseth, "Closing and Decommissioning Nuclear Power Reactors," 2012.

[2] United States Nuclear Regulatory Commission, "Protecting People and the Enviroment," 25 June 2012. [Online]. Available: http://www.nrc.gov/reading$\mathrm{rm} /$ doc-collections/fact-sheets/decommissioning.html. [Accessed December 2012].

[3] World Nuclear Association, "Decomissioning Nuclear Facilities," August 2012. [Online]. Available: http://www.world-nuclear.org/info/Nuclear-FuelCycle/Nuclear-Wastes/Decommissioning-Nuclear-Facilities/\#.UWWOo5NoZxY.

[4] Intel Labs Seattle, August 2012. [Online]. Available: http://www.seattle.intelresearch.net/WISP/.

[5] L. Chen, C. Ong, C. Neo, V. Varadan and V. Varadan, Microwave Electronics: Measurement and Materials Characterization, Chichester: John Wiley \& Sons Ltd, 2004, pp. 496-503.

[6] S. Georgakopoulos and S. Jiang, "Optimum Power Transmission of Wireless Sensors Embedded in Concrete," IEEE RFID, 2010.

[7] A. P. Sample, J. Braun, A. Parks and J. Smith, "Photovoltaic Enhanced UHF RFID Tag Antennas for Dual Purpose Energy Harvesting," in 2011 IEEE International Conferenceon RFID, 2011.

[8] F. Gasco, P. Ferabolli, J. Braun, J. Smith, P. Stickler and L. Deoto, "Wireless strain measurement for structural testing and health monitoring of cabon fiber composites," Composites: Part A, no. 42, pp. 1263-1274, 2011.

[9] E. Hoque, R. F. Dickerson and J. A. Stankovic, "Monitoring Body Positions and Movements During Sleep using WISPs," in Wireless Health, La Jolla, CA, 2010.

[10] E. Van Every, A. Deyhim and F. Faridazar, "Embedded Sensors for Life-time Monitoring Concrete," in Structural Health Monitoring on Intelligent Infrastructure, Zurich, Switzerland, 2009.

[11] C. A. Langton, M. G. Serrato, J. K. Blankenship and W. B. Griffin, "Savannah River Site R-Reactor Disassembly basin In-Situ Decomissioning," Savannah River Site National Laboratory, Phoeniz, AZ, 2010. 
[12] P. Chang and A. a. L. S. Flatau, "Review Paper: Health Monitoring of Civil Infrastructure," SAGE Journals, pp. 257-267, 2003.

[13] F. Yildiz, "Potential Ambient Energy-Harvesting Sources and Techniques," The Journal of Technology Studies, pp. 40-48.

[14] C. A. Balanis, Advenced Engineering Electromagnetics, john Wiley \& Sons, Inc, 1989.

[15] T. Frenzel and M. Koch, "Modelling Electromagnetic properties of typical building materials," in 2008 International Symposium on Electromagnetic CompatibilityEMC Europe, Hamburg,Germany, 2008.

[16] Hewlett Packard, "User's Manual HP 85070B Dielectric Probe Kit," June 1997. [Online]. Available: http://cp.literature.agilent.com/litweb/pdf/85070-90009.pdf. [Accessed 20 May 2012].

[17] E. G. Farr and C. A. Frost, "Impulse Propagation Measurements of the Dielectric Properties ofWater, Dry Sand, Moist Sand, and Concrete," Measurement Note 52, 1997.

[18] L. Sandrolini, U. Reggiani and A. Ogunsola, "Modelling the electrical properties of concrete for shielding effectiveness prediction," Journal pf Physics D: Applied Physics, vol. 40, pp. 5366-5372, 2007.

[19] D. Pena, R. Feick, H. Hristov and W. Grote, "Measurement and Modelling of Propagation Losses in Brick and Concrete Walls for the 900-MHz Band," IEEE Transactions on Antennas and Propagation, vol. 51, no. 1, pp. 31-39, Jan 2003.

[20] C. Balanis, Antenna Theory: Analysis and Design, 3rd ed., Hoboken: John Wiley \& Sons,Inc, 2005.

[21] Intel, November 2012. [Online]. Available: http://wisp.wikispaces.com/InstallingFirmware.

[22] P. N. Nikitin and K. Rao, "Performance Limitations of Passive UHF RFID Systems," in Proceedings of the IEEE Antennas and Propagation Symposium, 2006.

[23] WISP Intel, Nov 2012. [Online]. Available: https://github.com/wisp.

[24] A. Sample, D. Yeager, P. Powledge, A. Mamishev and J. Smith, "Design of and 
RFID-Based Battery-Free Programable Sensing Platform," IEEE Transactions on Instrumentation and Measurements, vol. 57, no. 11, pp. 2608-2615, Nov 2008.

[25] J. Landt, "The History of RFID," IEEE Potentials, vol. 24, no. 4, pp. 8-11, Oct-Nov 2005.

[26] EPC Global, October 2008. [Online]. Available:

http://www.gs1.org/gsmp/kc/epcglobal/uhfc1g2/uhfc1g2_1_2_0-standard20080511.pdf.

[27] Intel Labs Seattle, August 2012. [Online]. Available: http://www.seattle.intelresearch.net/WISP/.

[28] F. Gasco, P. Feraboli, J. Braun, J. Smith, P. Stickler and L. DeOto, "Wireless strain measurement for structural testing and health monitoring of carbon fiber composites," Composites:part A, vol. 42, pp. 1263-1274, May 2011.

[29] E. Hoque and J. A. Stankovic, "Monitoring Quantity and Quality of Sleeping Using WISPs," in IPSN'10, Stockholm, Sweden, 2010.

[30] J. Samseth, 2012. [Online]. Available: http://www.unep.org/yearbook/2012/.

[31] K. Shams and M. Ali, "Wireless Power Transmission to a Buried Sensor in Concrete," IEEE Transactions on Microwave Theory and Techniques, vol. 46, no. 12, pp. 2053-2060, Dec 1998.

[32] L. Rindorf and K. Jakobsen, "Small and Robust Antennas for Concrete Embedded Sensors," in Loughborough Antennas and Propagation Conference, 2009.

[33] K. Shams, M. Ali and A. Miah, "Characteristics of an Embedded Microstrip Patch Antenna for Wireless Infrastructure Health Monitoring," in IEEE Antennas and Propagation Society International Symposium, New Mexico, Albuquerque, 2010.

[34] X. Jin and M. Ali, "Embedded antennas in dry and saturated concrete for application in wireless sensor," Progress in Electromagnetics Research, vol. 102, pp. 197-211, 2010.

[35] Savannah River National Laboratory, "In-Situ Decommissioning of Large Nuclear Facilities," August 2012. [Online]. Available:

http://cab.srs.gov/library/meetings/2012/fb/09decom.pdf. 
[36] DOE Pulse Science and Technology Highlights from the DOE National Laboratories, "More than just a simple concrete job," 24 September 2012. [Online]. Available: http://www.ornl.gov/info/news/pulse/no372/story4.shtml. 